\title{
A renormalisation group method. V. A single renormalisation group step
}

\author{
David C. Brydges* and Gordon Slade*
}

November 25, 2014

\begin{abstract}
This paper is the fifth in a series devoted to the development of a rigorous renormalisation group method applicable to lattice field theories containing boson and/or fermion fields, and comprises the core of the method. In the renormalisation group method, increasingly large scales are studied in a progressive manner, with an interaction parametrised by a field polynomial which evolves with the scale under the renormalisation group map. In our context, the progressive analysis is performed via a finite-range covariance decomposition. Perturbative calculations are used to track the flow of the coupling constants of the evolving polynomial, but on their own perturbative calculations are insufficient to control error terms and to obtain mathematically rigorous results. In this paper, we define an additional non-perturbative coordinate, which together with the flow of coupling constants defines the complete evolution of the renormalisation group map. We specify conditions under which the non-perturbative coordinate is contractive under a single renormalisation group step. Our framework is essentially combinatorial, but its implementation relies on analytic results developed earlier in the series of papers. The results of this paper are applied elsewhere to analyse the critical behaviour of the 4-dimensional continuous-time weakly self-avoiding walk and of the 4-dimensional $n$-component $|\varphi|^{4}$ model. In particular, the existence of a logarithmic correction to mean-field scaling for the susceptibility can be proved for both models, together with other facts about critical exponents and critical behaviour.
\end{abstract}

\section{Introduction and main results}

\subsection{Background}

This paper is the fifth in a series devoted to the development of a rigorous renormalisation group method applicable to lattice field theories containing boson and/or fermion fields, and comprises the core of the method. Its immediate goal is to prepare for the application in [5, 6] to a specific supersymmetric field theory that is used to analyse the critical behaviour of the continuous-time weakly self-avoiding walk, and in particular to prove the existence of a logarithmic correction to

\footnotetext{
${ }^{*}$ Department of Mathematics, University of British Columbia, Vancouver, BC, Canada V6T 1Z2. E-mail: db5d@math.ubc.ca, slade@math.ubc.ca.
} 
the susceptibility in dimension 4. However, our approach is more general and applies more broadly including to the critical behaviour of the 4-dimensional $n$-component $\left|\varphi^{4}\right|$ model [9].

In the renormalisation group method, a multi-scale analysis is performed in which increasingly large scales are studied in a progressive manner, with an interaction parametrised by a field polynomial which evolves with the scale under renormalisation group transformations [40]. In our context, progressive integration is performed via a finite-range covariance decomposition [4, 15]. Perturbative calculations are used to track the flow of the coefficients, or coupling constants, of the evolving polynomial, but on their own perturbative calculations are insufficient to control error terms and to obtain mathematically rigorous results. In this paper, we employ another coordinate called $K$, in addition to the interaction polynomial $V$, for tracking the evolution under renormalisation group transformation. With this additional coordinate, we provide a framework that allows the error terms to be rigorously controlled. Our framework is essentially combinatorial, but its implementation relies on analytic results developed in earlier papers. An important feature of our method is that it respects supersymmetry, when this is present in the underlying model. Euclidean invariance is not manifest since our method relies on subdivisions of space into hypercubes. The use of such subdivisions has been universal in nonperturbative work on the renormalisation group, but recently [37] a manifestly Euclidean invariant method has been invented.

Some aspects of our approach, whose roots go back to [21], were presented in [14]. We draw on the approach of [13,16] for hierarchical models, but in a much extended and generalised form that applies to $\mathbb{Z}^{d}$. The idea of using a covariance decomposition to implement renormalisation goes back to [10,11]. Recent uses of the renormalisation group that bear some relation to our approach can be found in $[1,2,25,26,34$.

Different approaches to the renormalisation group include the block spin method used in [29 32, the phase space expansion method used in [28], and the approach of Bałaban (see e.g., [3], and [23] for a recent overview). These various methods are distinguished from each other according to how they combine perturbation theory with estimates on large deviations connected with large fields. Balaban's method is particularly powerful because it also applies to strong coupling problems where the action has degenerate minima. The books and major reviews [12, 14, 27, 33, 36, 38] give varied perspectives on renormalisation.

This paper is the culmination of the developments presented in parts I-IV [7, 18, 20] of the series and it relies on results from all four parts. A full assembly of parts I-V (and using also the result of [8]), is given for the 4-dimensional weakly self-avoiding walk in [5, 6], and for the 4-dimensional $|\varphi|^{4}$ model in [9]. To put the present paper in perspective, we briefly summarise the other papers in the series as they pertain to this one.

1. In part I [18], we present elements of the theory of Gaussian integration involving both boson and fermion fields, and develop norms and norm estimates for performing analysis with such Gaussian integrals. A renormalisation group step involves performing a Gaussian integral whose covariance is given by a generic term in the finite-range decomposition of an original covariance. In the present paper, we show how to obtain effective control on such an integration, so that error terms do not accumulate upon repeated integration.

2. In part II [19], we define and analyse the localisation operator Loc, which extracts from a functional of the fields a polynomial that captures the components of the functional which are relevant and marginal for the dynamical system defined by the renormalisation group. 
These are the components which must be accurately tracked, and this tracking leads to the flow of the coupling constants. In the present paper, we prove that the operator Loc achieves its purpose in the sense that the non-perturbative coordinate is contractive under the renormalisation group map. It is this contraction that prevents error terms from building up under successive renormalisation group steps.

3. In part III [7], we present a general description of perturbation theory, in which the polynomial $V_{j}$ at scale $j$ is replaced after a single Gaussian integral by a new polynomial $V_{\text {pt }}$. The polynomial $V_{\mathrm{pt}}$ is accurate to second order in the coupling constants but does not take into account error terms that have the potential to accumulate in repeated renormalisation group steps. In the present paper we show how to employ $V_{\mathrm{pt}}$ while preventing errors from accumulating.

4. In part IV [20], we prove nonperturbative estimates for the specific supersymmetric field theory studied in [5, 6]. The results include stability estimates for the interaction, proof of accuracy of the perturbative calculations of part III, estimates on Gaussian expectations, and a crucial contraction estimate which implements the achievements of the operator Loc. The estimates of part IV provide an essential input for the present paper.

5. As an application and dénoument, in [5, 6] we obtain a statement of infrared asymptotic freedom for the 4-dimensional weakly self-avoiding walk, and use it to prove the existence of a logarithmic correction to mean-field scaling for the sucsceptibility and $|x|^{-2}$ decay for the critical two-point function. The analysis of [5, 6] combines the results of parts I-V with the main result of [8] to analyse the infinite-dimensional dynamical system arising from repeated application of the renormalisation group. A further application to the 4-dimensional $n$-component $\varphi^{4}$ model is given in [9].

Throughout the paper, we concentrate on the case of dimension $d=4$. Before stating our main results in Section 1.8, we first introduce the language and concepts needed for their formulation, as well as the norms used in their statement.

\subsection{Polymers and local algebras of forms}

Let $L \geq 3$ and $N \geq 1$ be integers, and let $\Lambda=\mathbb{Z}^{d} /\left(L^{N} \mathbb{Z}^{d}\right)$ for fixed dimension $d>0$. We write $|\cdot|$ for the $\ell_{\infty}$ distance on both $\mathbb{Z}^{d}$ and the torus $\Lambda$. Since $N$ and $\Lambda$ are determined by each other we make $\Lambda$ the primary object and write $N=N(\Lambda)$. Our results concern the renormalisation group in both finite volume $\Lambda$ and the infinite volume $\mathbb{Z}^{d}$. To cover both cases we use the symbol $\mathbb{V}$ whose values are $\Lambda$ or $\mathbb{Z}^{d}$, and we set $N(\mathbb{V})=\infty$ for $\mathbb{V}=\Lambda$. To allow for the study of the two-point function, two particular points $a, b$ are fixed in $\mathbb{Z}^{d}$. We assume $a, b$ have distinct images in $\Lambda$, under the projection $x \mapsto x \bmod \left(L^{N} \mathbb{Z}^{d}\right)$, and their images are also called $a, b$ so we can refer to the two distinguished points in $\mathbb{V}$. They are called observable points. The following definition is basic to our setup.

Definition 1.1. (a) Blocks. For each $j \in \mathbb{N}_{0}$ the lattice $\mathbb{Z}^{d}$ is paved in a natural way by disjoint $d$-dimensional cubes of side $L^{j}$. The cube that contains the origin at the corner has the form

$$
\left\{x \in \Lambda:|x|_{\infty}<L^{j}\right\}
$$


and all other cubes are translates of this one by vectors in $L^{j} \mathbb{Z}^{d}$. Similarly, for $j=0,1, \ldots, N(\Lambda)$, the torus $\Lambda$ is paved in a natural way by $L^{N-j}$ disjoint $d$-dimensional cubes of side $L^{j}$. We call these cubes $j$-blocks, or blocks for short and let $\mathcal{B}_{j}=\mathcal{B}_{j}(\mathbb{V})$ denote the set of $j$-blocks. The integer $j$ is called a scale.

(b) Polymers. A union of blocks in $\mathcal{B}_{j}$ is called a polymer (at scale $j$ ), and the set of polymers at scale $j$ is denoted $\mathcal{P}_{j}=\mathcal{P}_{j}(\mathbb{V})$. The empty union is included: $\varnothing \in \mathcal{P}_{j}$. For $X \in \mathcal{P}_{j}, \mathcal{B}_{j}(X)$ denotes the set of blocks $B \in \mathcal{B}_{j}$ with $B \subset X$. The size $|X|_{j}$ of $X \in \mathcal{P}_{j}$ is the number of $j$-blocks in $X$, i.e., $|X|_{j}$ is the cardinality of $\mathcal{B}_{j}(X)$. We define $\mathcal{P}_{*}=\sqcup_{j} \mathcal{P}_{j}\left(\mathbb{Z}^{d}\right)$. In particular, an element $X$ of $\mathcal{P}_{*}$ has a scale $j(X)$.

(c) Connectivity. A nonempty subset $X \subset \Lambda$ is said to be connected if for any two points $x, x^{\prime} \in X$ there exists points $x_{i} \in X(i=0,1, \ldots, n)$ with $\left|x_{i+1}-x_{i}\right|_{\infty}=1, x_{0}=x$ and $x_{n}=x^{\prime}$. The set of connected polymers in $\mathcal{P}_{j}$ is denoted $\mathcal{C}_{j}=\mathcal{C}_{j}(\mathbb{V})$. The null set $\varnothing$ is not in $\mathcal{C}_{j}$. We say that two polymers $X, Y$ do not touch if $\min \left\{|x-y|_{\infty}: x \in X, y \in Y\right\}>1$. A polymer can be decomposed into connected components that do not touch; we write $\operatorname{Comp}(X)$ for the set of connected components of $X$.

The basic setting for our analysis is detailed in [20, Section 1.1], and we maintain the same setting and notation here, but now allow infinite volume as well as finite volume. In brief, we have a complex boson field $\phi: \Lambda \rightarrow \mathbb{C}$ with its complex conjugate $\bar{\phi}$, a pair of conjugate fermion fields $\psi, \bar{\psi}$, and a constant complex observable boson field $\sigma \in \mathbb{C}$ with its complex conjugate $\bar{\sigma}$. The fermion field is given in terms of the 1 -forms $d \phi_{x}$ by $\psi_{x}=\frac{1}{\sqrt{2 \pi i}} d \phi_{x}$ and $\bar{\psi}_{x}=\frac{1}{\sqrt{2 \pi i}} d \bar{\phi}_{x}$, where we fix some square root of $2 \pi i$. We work with an algebra $\mathcal{N}$ which is defined in terms of a direct sum decomposition

$$
\mathcal{N}=\mathcal{N}^{\varnothing} \oplus \mathcal{N}^{a} \oplus \mathcal{N}^{b} \oplus \mathcal{N}^{a b}
$$

Elements of $\mathcal{N}^{\varnothing}$ are given by finite linear combinations of products of an even number of fermion fields with coefficients that are complex-valued functions of the boson fields. This restriction to forms of even degree results in a commutative algebra. Elements of $\mathcal{N}^{a}, \mathcal{N}^{b}, \mathcal{N}^{a b}$ are respectively given by elements of $\mathcal{N}^{\varnothing}$ multiplied by $\sigma$, by $\bar{\sigma}$, and by $\sigma \bar{\sigma}$. For example, $\phi_{x} \bar{\phi}_{y} \psi_{x} \bar{\psi}_{x} \in \mathcal{N}^{\varnothing}$, and $\sigma \bar{\phi}_{x} \in \mathcal{N}^{a}$. There are canonical projections $\pi_{\alpha}: \mathcal{N} \rightarrow \mathcal{N}^{\alpha}$ for $\alpha \in\{\varnothing, a, b, a b\}$. We use the abbreviation $\pi_{*}=1-\pi_{\varnothing}=\pi_{a}+\pi_{b}+\pi_{a b}$. The algebra $\mathcal{N}$ is discussed further around [19, (1.60)]. There $\mathcal{N}$ is written $\mathcal{N} / \mathcal{I}$, but to simplify the notation we write $\mathcal{N}$ here instead. The quotient space notation reflects our policy of writing arbitrary functions of $\sigma, \bar{\sigma}$ and identifying any such function with the sum of the constant, $\sigma, \bar{\sigma}$ and $\sigma \bar{\sigma}$ terms in its formal power series expansion in $\sigma, \bar{\sigma}$. The parameter $p_{\mathcal{N}}$ which appears in its definition is a measure of the smoothness of elements of $\mathcal{N}$ (see [18, Section 2.1]); its precise value is unimportant as long as it is fixed with $p_{\mathcal{N}} \geq 10$ (the value "10" is required for Lemma 2.4 below). Constants in estimates are permitted to depend on $p_{\mathcal{N}}$, and this is unimportant.

In [18, (3.15), (3.38)], $\mathcal{N}(X)$ is defined to be the algebra of differential forms that depend only on fields with spatial labels in $X$, where $X$ is a subset of $\Lambda$. In this paper the argument $X$ of $\mathcal{N}(X)$ is a subset of $\mathbb{V}$, which is $\Lambda$ or $\mathbb{Z}^{d}$, and $\mathcal{N}(X)$ consists of differential forms of even degree generated by monomials in $\psi, \bar{\psi}$ with spatial labels in $X$, so that $\mathcal{N}(X)$ is commutative. We also define the commutative algebra

$$
\mathcal{N}(\mathbb{V})=\bigcup_{X \text { finite, } X \subset \mathbb{V}} \mathcal{N}(X)
$$


For $\mathbb{V}=\Lambda$ or $\mathbb{V}=\mathbb{Z}^{d}$ we write $\mathcal{N}=\mathcal{N}(\mathbb{V})$. Note that $\mathcal{N}(X)$ is a subalgebra of $\mathcal{N}(Y)$ when $X$ is a subset of $Y$.

In the notation of [18, Section 3.2], for $X \subset \Lambda$, an element of $\mathcal{N}(X)$ has the form

$$
F=\sum_{y \in \vec{\Lambda}^{*}} \frac{1}{y !} F_{y} \psi^{y} .
$$

The sum is over sequences $y=(x, \bar{x})$, with each of $x=\left(x_{1}, \ldots, x_{p}\right)$ and $\bar{x}=\left(\bar{x}_{1}, \ldots, \bar{x}_{q}\right)$ a sequence in $X$, with $\psi^{y}=\psi_{x_{1}} \cdots \psi_{x_{p}} \bar{\psi}_{\bar{x}_{1}} \cdots \bar{\psi}_{\bar{x}_{q}}$, and with $y !=p ! q !$. The coefficient $F_{y}$ is a complex valued function of $(\phi, \sigma)$ in $\mathbb{C}^{\mathbb{V}} \times \mathbb{C}$ such that $F_{y}\left(\phi^{\prime}, \sigma\right)=F_{y}(\phi, \sigma)$ when $\left.\phi^{\prime}\right|_{X}=\left.\phi\right|_{X}$. The coefficients $F_{y}$ are zero when the sequence $y$ has odd length. As a function of $\sigma, F_{y}$ has the form $\alpha+\beta \sigma+\gamma \bar{\sigma}+\delta \sigma \bar{\sigma}$, but $\beta=\delta=0$ when $X$ does not contain $a$ and $\gamma=\delta=0$ when $X$ does not contain $b$. To understand this, one should regard $\sigma$ as associated to the point $a$, and $\bar{\sigma}$ to the point $b$, and then the conditions say that an element $F$ of $\mathcal{N}(X)$ depends only on fields in $X$.

Let $\mathcal{U}$ denote the set of $2 d$ nearest neighbours of the origin in $\mathbb{Z}^{d}$. For $e \in \mathcal{U}$, we define the finite difference operator $\nabla^{e} \phi_{x}=\phi_{x+e}-\phi_{x}$, and the Laplacian $\Delta_{\mathbb{Z}^{d}}=-\frac{1}{2} \sum_{e \in \mathcal{U}} \nabla^{-e} \nabla^{e}$. Important examples of forms are:

$$
\begin{gathered}
\tau_{x}=\phi_{x} \bar{\phi}_{x}+\psi_{x} \bar{\psi}_{x}, \quad \tau_{\nabla \nabla, x}=\frac{1}{2} \sum_{e \in \mathcal{U}}\left(\left(\nabla^{e} \phi\right)_{x}\left(\nabla^{e} \bar{\phi}\right)_{x}+\left(\nabla^{e} \psi\right)_{x}\left(\nabla^{e} \bar{\psi}\right)_{x}\right) \\
\tau_{\Delta, x}=\frac{1}{2}\left((-\Delta \phi)_{x} \bar{\phi}_{y}+\phi_{x}(-\Delta \bar{\phi})_{y}+(-\Delta \psi)_{x} \bar{\psi}_{y}+\psi_{x}(-\Delta \bar{\psi})_{y}\right) .
\end{gathered}
$$

Let $\mathcal{Q}$ denote the vector space of polynomials of the form

$$
V=V_{\varnothing}+V_{a}+V_{b}+V_{a b}
$$

where

$$
\begin{gathered}
V_{\varnothing}=g \tau^{2}+\nu \tau+z \tau_{\Delta}+y \tau_{\nabla \nabla}, \quad V_{a}=\lambda_{a} \sigma \bar{\phi}, \quad V_{b}=\lambda_{b} \bar{\sigma} \phi, \quad V_{a b}=q_{a b} \bar{\sigma} \sigma, \\
\lambda_{a}=-\lambda^{a} \mathbb{1}_{a}, \quad \lambda_{b}=-\lambda^{b} \mathbb{1}_{b}, \quad q_{a b}=-\frac{1}{2}\left(q^{a} \mathbb{1}_{a}+q^{b} \mathbb{1}_{b}\right),
\end{gathered}
$$

$g, \nu, y, z, \lambda^{a}, \lambda^{b}, q^{a}, q^{b} \in \mathbb{C}$, and the indicator functions are defined by the Kronecker delta $\mathbb{1}_{a, x}=$ $\delta_{a, x}$. For $X \subset \Lambda$, we write

$$
V(X)=\sum_{x \in X} V_{x}
$$

Elements $V$ of $\mathcal{Q}$ are polynomials with eight independent coefficients, so $\mathcal{Q}$ is isomorphic to $\mathbb{C}^{8}$ and this identification is sometimes useful. The polynomial $V$ has symmetries which are inherited by the field theory to be defined below in terms of $V$. To discuss these symmetries, an automorphism $E: \Lambda \rightarrow \Lambda$ is an injective map from $\Lambda$ to $\Lambda$ under which nearest-neighbour points are mapped to nearest-neighbour points under both the map and its inverse. Translations and reflections that preserve $\Lambda$ are examples of automorphisms. The action of an automorphism $E: \Lambda \rightarrow \Lambda$ as a map from $\mathcal{N}(\Lambda)$ to itself is defined in [19, (1.28)]. The polynomial $V_{\varnothing}$ is Euclidean covariant, in the sense that for any automorphism $E, E\left(V_{\varnothing, x}\right)=V_{\varnothing, E x}$. Also, $V_{x}$ is gauge invariant and $V_{\varnothing, x}$ is supersymmetric, where these two terms are defined for elements of $\mathcal{N}$ in [7, Section 5.2]. 


\subsection{Covariance decomposition}

Given $m^{2}>0$, let $C=\left(-\Delta_{\Lambda}+m^{2}\right)^{-1}$. As explained in more detail in [20, Section 1.1.1], the covariance $C$ has a finite-range decomposition $C=C_{1}+\cdots C_{N-1}+C_{N, N}$ [4, 15. The expectation $\mathbb{E}_{C}$ denotes the combined bosonic-fermionic Gaussian integration on $\mathcal{N}$, with covariance $C$, defined in [18, Section 2.4]. The expectation can be performed successively, using

$$
\mathbb{E}_{C} \theta=\mathbb{E}_{N} \theta \circ \mathbb{E}_{N-1} \theta \circ \cdots \circ \mathbb{E}_{1} \theta
$$

where $\mathbb{E}_{j}$ is the expectation corresponding to the $j^{\text {th }}$ covariance, and $\theta$ denotes a type of convolution. More precisely, we define the map $\theta: \mathcal{N}(\mathbb{V}) \rightarrow \mathcal{N}\left(\mathbb{V} \sqcup \mathbb{V}^{\prime}\right)$ by making the replacement in an element of $\mathcal{N}$ of $\phi$ by $\phi+\xi, \bar{\phi}$ by $\bar{\phi}+\bar{\xi}, \psi$ by $\psi+\eta$, and $\bar{\psi}$ by $\bar{\psi}+\bar{\xi}$. In applying $\mathbb{E}_{j+1} \theta$, the fields $\xi, \bar{\xi}, \eta, \bar{\eta}$ are integrated out by $\mathbb{E}_{j}$, with $\phi, \bar{\phi}, \psi, \bar{\psi}$ kept fixed. The expectation $\mathbb{E}_{C}$ can be obtained as the special case of (1.11) resulting from setting $\xi=\bar{\xi}=\eta=\bar{\eta}=0$ in $\mathbb{E}_{N} \theta$.

We assume that the covariance decomposition obeys the estimates listed and discussed in [20, Section 1.3.1]. In particular, for [20, (1.71)], we restrict $m^{2}$ to lie in a small interval $[0, \delta]$ when considering $C_{j}$ with $j<N$, but make the further restriction $m^{2} \in\left[\delta L^{-2(N-1)}, \delta\right]$ for $C_{N, N}$. The covariances obey the finite-range property that $C_{j}(x, y)=0$ for $|x-y| \geq \frac{1}{2} L^{j}$, for each scale $j$. These properties are established for the covariance decomposition of [4] in [7].

In analogy with ordinary Gaussian random variables, there is an independence consequence of the finite-range property, called the factorisation property of the expectation. The latter states that if $X_{1}, \ldots, X_{n} \in \mathcal{P}_{j+1}(\Lambda)$ do not touch each other, and if $F_{m}\left(X_{m}\right) \in \mathcal{N}\left(X_{m}\right)$ for each $m$, then

$$
\mathbb{E}_{j+1} \theta \prod_{m=1}^{n} F_{m}\left(X_{m}\right)=\prod_{m=1}^{n} \mathbb{E}_{j+1} \theta F_{m}\left(X_{m}\right) .
$$

This factorisation property is a consequence of [18, Proposition 2.7]. It plays an important role.

\subsection{Perturbative and non-perturbative coordinates}

As in [20, (1.22)], the interaction is defined, for $V \in \mathcal{Q}, B \in \mathcal{B}_{j}$ and $X \in \mathcal{P}_{j}$, by

$$
I_{j}(V, B)=e^{-V(B)}\left(1+W_{j}(V, B)\right), \quad I_{j}(V, X)=\prod_{B \in \mathcal{B}_{j}(X)} I_{j}(V, B),
$$

where $W_{j}$ is a certain non-local polynomial in the fields, which is an explicit quadratic function of $V$ discussed in detail in [20, Section 1.1.3. In the present paper, we rely on properties of $I$ proved in [20] and the specifics of its definition play a minor role.

Recall the function $V_{\mathrm{pt}}: \mathcal{Q} \rightarrow \mathcal{Q}$ defined in [7, (3.23)] and explained in [7, Section 2]. In [7, Proposition 2.1], we show that

$$
\mathbb{E}_{j+1} \theta I_{j}(V, \Lambda) \approx I_{j+1}\left(V_{\mathrm{pt}}, \Lambda\right),
$$

where the approximation is accurate up to and including second order, as formal power series in the coupling constants. Under this approximate perturbative calculation, the effect of a single expectation is captured by the map $V \mapsto V_{\mathrm{pt}}$, and we refer to $V$ as the perturbative coordinate. We introduce a non-perturbative coordinate $K$ which accurately tracks all the errors in the approximation (1.14). For this, the following definition is needed. 
Definition 1.2. Circle product. Given $F, G: \mathcal{P}_{j} \rightarrow \mathcal{N}$, we define $F \circ G: \mathcal{P}_{j} \rightarrow \mathcal{N}$ by

$$
(F \circ G)(X)=\sum_{Y \in \mathcal{P}_{j}(X)} F(Y) G(X \backslash Y) \quad\left(X \in \mathcal{P}_{j}\right) .
$$

This circle product is commutative and associative.

The circle product depends on $j$ but this is left implicit in the notation. All functions $F: \mathcal{P}_{j} \rightarrow$ $\mathcal{N}$ that we consider are required to obey $F(\varnothing)=1$. The sum in (1.15) includes the degenerate terms $Y=\varnothing, X$ (in particular, $(F \circ G)(\varnothing)=F(\varnothing) G(\varnothing)=1$ ). The identity element for the circle product is $\mathbb{1}_{\varnothing}$, defined by setting $\mathbb{1}_{\varnothing}(X)=1$ if $X=\varnothing$ and $\mathbb{1}_{\varnothing}(X)=0$ otherwise. From (1.11), we obtain

$$
\mathbb{E}_{C} \theta I_{0}(V, \Lambda)=\mathbb{E}_{C} \theta\left(I_{0} \circ \mathbb{1}_{\varnothing}\right)(\Lambda)=\mathbb{E}_{N} \theta \circ \mathbb{E}_{N-1} \theta \circ \cdots \circ \mathbb{E}_{1} \theta\left(I_{0} \circ \mathbb{1}_{\varnothing}\right)(\Lambda) .
$$

Let $\mathcal{Q}^{(0)}$ be the subspace of $\mathcal{Q}$ with $y=q^{a}=q^{b}=0$. Let $j<N(\mathbb{V})$, let $q_{j} \in \mathbb{C}$, let $V_{j} \in \mathcal{Q}^{(0)}$, and let $K_{j}: \mathcal{P}_{j} \rightarrow \mathcal{N}$. The renormalisation group map $\mathrm{RG}=\mathrm{RG}_{j}$ is a description of the action of $\mathbb{E}_{j+1} \theta$ as a map RG: $\left(q_{j}, V_{j}, K_{j}\right) \mapsto\left(q_{j+1}, V_{j+1}, K_{j+1}\right)$, with $q_{j+1} \in \mathbb{C}, V_{j+1} \in \mathcal{Q}^{(0)}$, and $K_{j+1}: \mathcal{P}_{j+1} \rightarrow \mathcal{N}$, such that

$$
e^{q_{j} \sigma \bar{\sigma}} \mathbb{E}_{j+1} \theta\left(I_{j}\left(V_{j}\right) \circ K_{j}\right)(\Lambda)=e^{q_{j+1} \sigma \bar{\sigma}}\left(I_{j+1}\left(V_{j+1}\right) \circ K_{j+1}\right)(\Lambda) .
$$

This allows (1.16) to be evaluated iteratively. In particular, the flow of $q$ under repeated applications of the renormalisation group map turns out to be central to the proof obtaining the decay of the critical two-point function of the continuous-time weakly self-avoiding walk in [5]. By dividing (1.17) by $e^{q_{j} \sigma \bar{\sigma}}$ and setting $\delta q_{j+1}=q_{j+1}-q_{j}$, we obtain the equivalent equation

$$
\mathbb{E}_{j+1} \theta\left(I_{j}\left(V_{j}\right) \circ K_{j}\right)(\Lambda)=e^{\delta q_{j+1} \sigma \bar{\sigma}}\left(I_{j+1}\left(V_{j+1}\right) \circ K_{j+1}\right)(\Lambda) .
$$

Thus we can regard RG as the map

$$
\mathrm{RG}_{j}:\left(V_{j}, K_{j}\right) \mapsto\left(\delta q_{j+1}, V_{j+1}, K_{j+1}\right)
$$

The existence of a map obeying (1.17) is easy: there are $q_{j+1}, K_{j+1}$ that solve this equation for any choice of $V_{j+1}$, and they are not unique. An example is given in Section 1.5 below. It is much harder to choose the map RG and a Banach space in which $K_{j+1}$ does not grow in norm under iteration of the renormalisation group map, and the main achievement of the present paper is to exhibit such a choice.

\subsection{Simplified construction of $K_{1}$}

For illustrative purposes, we now provide an example of a simplified construction of $K_{1}$ from $\left(V_{0}, K_{0}\right)=\left(V_{0}, \mathbb{1}_{\varnothing}\right)$. The idea in this section is used in Section 5.1 below, but the complete construction of RG requires a better (but less simple) choice of $K_{+}$than the one in the example.

The following elementary lemma, which relates the circle product and binomial expansion, is useful here and also later. It uses notation discussed in more detail around (1.29). Namely, given $F: \mathcal{B}_{j} \rightarrow \mathcal{N}$ and $X \in \mathcal{P}_{j}$, we write $F^{X}=F(X)=\prod_{B \in \mathcal{B}_{j}(X)} F(B)$. 


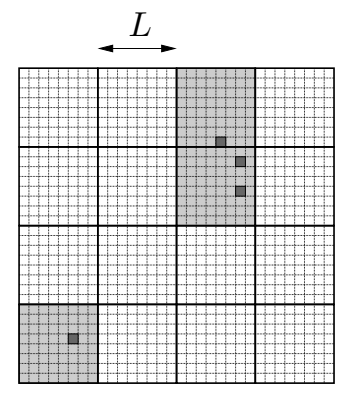

Figure 1: The four small dark squares represent a polymer in $\mathcal{P}_{0}$, and the three larger shaded squares represent its closure in $\mathcal{P}_{1}$.

Lemma 1.3. For $F_{1}, F_{2}: \mathcal{B}_{j} \rightarrow \mathcal{N}$ and $X \in \mathcal{P}_{j}$,

$$
\left(F_{1}+F_{2}\right)^{X}=\left(F_{1} \circ F_{2}\right)(X) .
$$

Proof. By (1.29), followed by expansion of the product and application of (1.15), we find that

$$
\left(F_{1}+F_{2}\right)^{X}=\prod_{B \in \mathcal{B}_{j}(X)}\left(F_{1}+F_{2}\right)(B)=\sum_{Y \in \mathcal{P}_{j}(X)} F_{1}^{Y} F_{2}^{X \backslash Y}=\left(F_{1} \circ F_{2}\right)(X),
$$

and the proof is complete.

We also need the following definition, which is depicted in Figure 1.

Definition 1.4. The closure $\bar{X}$ of $X \in \mathcal{P}_{j}$ is the smallest $Y \in \mathcal{P}_{j+1}$ such that $X \subset Y$. Given $U \in \mathcal{P}_{j+1}$, we write

$$
\overline{\mathcal{P}}_{j}(U)=\left\{X \in \mathcal{P}_{j} \mid \bar{X}=U\right\}
$$

The following proposition provides an example of a construction of $K_{1}$ from the pair $I_{0}$ and $K_{0}=\mathbb{1}_{\varnothing}$, for arbitrary choice of $V_{0}, V_{1}$ each with $q_{a b}=0$.

Proposition 1.5. For any $V_{0}, V_{1} \in \mathcal{Q}$, each with $q_{a b}=0$,

$$
\mathbb{E}_{1}\left(I_{0}\left(V_{0}\right) \circ \mathbb{1}_{\varnothing}\right)(\Lambda)=\left(I_{1}\left(V_{1}\right) \circ \tilde{K}_{1}\right)(\Lambda)
$$

where

$$
\tilde{K}_{1}(U)=\sum_{X \in \overline{\mathcal{P}}_{0}(U)} I_{1}^{U \backslash X} \mathbb{E}_{C_{1}} \delta I_{1}^{X}
$$

with $\delta I_{1}^{X}=\prod_{x \in X}\left(\theta I_{0}(x)-I_{1}(x)\right)$.

Proof. For $X \in \mathcal{P}_{0}$, let $\delta I_{1}^{X}=\prod_{x \in X}\left(\theta I(x)-I_{1}(x)\right)$; this depends on $\phi_{1}, \bar{\phi}_{1}, \psi_{1}, \bar{\psi}_{1}$ via $I_{1}$, as well as on the fields $\phi_{0}=\phi_{1}+\xi_{1}, \bar{\phi}_{0}=\bar{\phi}_{1}+\bar{\xi}_{1}, \psi_{0}=\psi_{1}+\eta_{1}, \bar{\psi}_{0}=\bar{\psi}_{1}+\bar{\eta}_{1}$ via $\theta I$. The integration 
implied by $\mathbb{E}_{1} \theta$ integrates out only the fluctuation fields $\xi_{1}, \bar{\xi}_{1}, \eta_{1}, \bar{\eta}_{1}$, leaving dependence on the scale-1 fields only. Thus we obtain, using Lemma 1.3 for the third equality,

$$
\begin{aligned}
\mathbb{E}_{1} \theta\left(I_{0} \circ \mathbb{1}_{\varnothing}\right)(\Lambda) & =\mathbb{E}_{1} \theta I_{0}(\Lambda)=\mathbb{E}_{1}\left(I_{1}+\delta I_{1}\right)^{\Lambda} \\
& =\mathbb{E}_{1}\left(I_{1} \circ \delta I_{1}\right)(\Lambda)=\left(I_{1} \circ \mathbb{E}_{1} \delta I_{1}\right)(\Lambda) .
\end{aligned}
$$

The above circle products are at scale 0. Using (1.24) for the last equality, we obtain

$$
\begin{gathered}
\left(I_{1} \circ \mathbb{E}_{1} \theta I\right)(\Lambda)=\sum_{X \in \mathcal{P}_{0}} I_{1}^{\Lambda \backslash X} \mathbb{E}_{1} \delta I_{1}^{X}=\sum_{U \in \mathcal{P}_{1}} \sum_{X \in \overline{\mathcal{P}}_{0}(U)} I_{1}^{\Lambda \backslash X} \mathbb{E}_{1} \delta I_{1}^{X} \\
=\sum_{U \in \mathcal{P}_{1}} I_{1}^{\Lambda \backslash U} \tilde{K}_{1}(U)=\left(I_{1} \circ \tilde{K}_{1}\right)(\Lambda),
\end{gathered}
$$

where the circle product on the right-hand side is at scale 1 . This completes the proof.

An important fact is that $\tilde{K}_{1}$ has a certain component factorisation property. For example, if $U \in \mathcal{P}_{1}$ has connected components $U_{1}, U_{2}$, then with the help of Figure 1 it is straightforward to check that the factorisation property (1.12) of the expectation implies that $\tilde{K}(U)=\tilde{K}\left(U_{1}\right) \tilde{K}\left(U_{2}\right)$. We make a formal definition of the component factorisation property in the next section.

\subsection{Setting for non-perturbative coordinate}

We now define the basic setting for the non-perturbative coordinate $K: \mathcal{P}_{j} \rightarrow \mathcal{N}$, including the spaces $\mathcal{C} \mathcal{K}_{j}$ and $\mathcal{K}_{j}$.

We say that a function $K: \mathcal{P}_{j} \rightarrow \mathcal{N}$ is Euclidean covariant if $E(K(X))=K(E X)$ for all polymers $X \in \mathcal{P}_{j}$ and all automorphisms $E$ of $\mathbb{V}$. We say that $K$ is gauge invariant (supersymmetric) if $K(X)$ is gauge invariant (supersymmetric) for all $X$ in $\mathcal{P}_{j}$; these two terms are defined for elements of $\mathcal{N}$ in [7, Section 5.2]. We say that $K$ has zero constant part if the result of setting $\phi=0$ and $\psi=0$ in $K(X)$ is zero for all non-empty polymers $X$. We need the following two definitions.

Definition 1.6. Small sets. A polymer $X \in \mathcal{P}_{*}$ is said to be a small set if $|X|_{j(X)} \leq 2^{d}$ and $X \in \mathcal{C}_{j(X)}$. Let $\mathcal{S}_{j}$ be the set of all small sets in $\mathcal{P}_{j}$. The small set neighbourhood of $X \in \mathcal{P}_{*}$ is defined by

$$
X^{\square}=\bigcup_{Y \in \mathcal{S}_{j(X)}: X \cap Y \neq \varnothing} Y .
$$

For the next definition, we define the coalescence scale $j_{a b}$ by

$$
j_{a b}=\left\lfloor\log _{L}(2|a-b|)\right\rfloor .
$$

Definition 1.7. For $j \leq N(\mathbb{V})$ with $j<\infty$, let $\mathcal{C} \mathcal{K}_{j}=\mathcal{C} \mathcal{K}_{j}(\mathbb{V})$ denote the complex vector space of functions $K: \mathcal{C}_{j}(\mathbb{V}) \rightarrow \mathcal{N}(\mathbb{V})$ with the properties:

- Field Locality: For all $X \in \mathcal{C}_{j}(\mathbb{V}), K(X) \in \mathcal{N}\left(X^{\square}\right)$. Also, (i) $\pi_{a} K(X)=0$ unless $a \in X$, (ii) $\pi_{b} K(X)=0$ unless $b \in X$, and (iii) $\pi_{a b} K(X)=0$ unless $a \in X$ and $b \in X^{\square}$ or vice versa, and $\pi_{a b} K(X)=0$ if $X \in \mathcal{S}_{j}$ and $j<j_{a b}$. 
- Symmetry: (i) $K$ is gauge invariant; (ii) $\pi_{\varnothing} K$ is supersymmetric and has no constant part; (iii) $\pi_{\varnothing} K$ is Euclidean covariant.

Let $\mathcal{K}_{j}=\mathcal{K}_{j}(\mathbb{V})$ be the complex vector space of functions $K: \mathcal{P}_{j}(\mathbb{V}) \rightarrow \mathcal{N}(\mathbb{V})$ which have the properties listed above and in addition

- Component Factorisation: for all polymers $X, K(X)=\prod_{Y \in \operatorname{Comp}(X)} K(Y)$.

Every element of $\mathcal{K}_{j}$ determines an element of $\mathcal{C} \mathcal{K}_{j}$ by restriction to connected sets, and every element of $\mathcal{C K}_{j}$ determines an element of $\mathcal{K}_{j}$ by the factorisation condition. The same symbol is used for both elements related by this correspondence. Under this correspondence, $\mathbb{1}_{\varnothing} \in \mathcal{K}_{j}$ becomes $0 \in \mathcal{C K}_{j}$, because the empty set is not a connected set.

Let $\mathcal{B K}_{j}=\mathcal{B K}_{j}(\mathbb{V})$ denote the set of functions $F: \mathcal{B}_{j} \rightarrow \mathcal{N}$ which obey the field locality and symmetry conditions of Definition 1.7. Given $F: \mathcal{B}_{j} \rightarrow \mathcal{N}$ we extend $F$ to $\mathcal{P}_{j}$ by

$$
F(X)=F^{X}=\prod_{B \in \mathcal{B}_{j}(X)} F(B) \quad\left(X \in \mathcal{P}_{j}\right)
$$

The appearance of the set $X$ as an exponent introduces our convention that such exponents signal functions that factorise over blocks. Using (11.29), an element $F \in \mathcal{B K}_{j}$ extends to an element $F \in \mathcal{K}_{j}$. An important use of $\mathcal{B} \mathcal{K}_{j}$ is the map $I_{j}: \mathcal{Q} \rightarrow \mathcal{B} \mathcal{K}_{j}(\Lambda)$ defined in (1.13).

The individual properties of Definition 1.7 play different roles in our analysis. The property of field locality is of fundamental importance and its preservation under iteration of the renormalisation group map relies on the finite-range property of the covariance decomposition via (1.12), as illustrated in Section 1.5 above. The symmetry properties are enjoyed by any $V_{j} \in \mathcal{Q}^{(0)}$, and the symmetry assumption on $K$ ensures that the effect of $K_{j}$ on the construction of $V_{j+1}$ is such that these symmetries are inherited from $V_{j}$ by $V_{j+1}$, and in particular that $V_{j+1}$ does not contain additional terms not present in $\mathcal{Q}^{(0)}$. It is possible to relax the assumption of supersymmetry by a suitable enlargement of $\mathcal{Q}$. For example, in the analysis of the $|\varphi|^{4}$ model in [9] we forego supersymmetry in Definition 1.7 at the cost of including an additional constant term in $\mathcal{Q}$; this is discussed in Remark 6.3 below.

\subsection{Definition of norms}

We use specific norms as detailed in this section. This particular specification is made so that we can apply estimates on $I$ (e.g., in Section 3.3) and an important contraction property (namely Proposition [5.5); these results are proved in [20]. It also paves the way for applications of our results in [5, 6, 9]. However, accepting the results of [20], the majority of this paper can be read without knowing what the norms are, beyond the facts that the norm of a product is less than the product of the norms, and the norm of an expectation is less than the expectation of the norm.

\subsubsection{Parameters}

We use the norms and regulators for $\mathcal{N}$ defined in [20, Section 1.1.6], including the $\Phi$ norm on test functions, the $\tilde{\Phi}$ norm on boson fields, the $T_{\phi}$ semi-norm on $\mathcal{N}$. The parameters $\mathfrak{h}=\ell_{j}$ and $\mathfrak{h}=h_{j}$ for these norms are specified in [20, Section 1.3.2] and we repeat the definition of these 
parameters here. They depend, in particular, on two numbers $\tilde{g}_{j}$ and $\tilde{g}_{j+1}$, which we assume can be taken to be as small as desired (uniformly in $j$, and depending on $L$ ), and which obey

$$
\frac{1}{2} \tilde{g}_{j+1} \leq \tilde{g}_{j} \leq 2 \tilde{g}_{j+1}
$$

This permits us to apply results from [20] which rely on (1.30). The parameters $\mathfrak{h}$ are given in terms of a (large) $L$-dependent constant $\ell_{0}$ and a (small) universal constant $k_{0}$ by

$$
\begin{gathered}
\mathfrak{h}_{j}= \begin{cases}\ell_{0} L^{-j[\phi]} & \mathfrak{h}=\ell \\
k_{0} \tilde{g}_{j}^{-1 / 4} L^{-j d / 4} & \mathfrak{h}=h,\end{cases} \\
\mathfrak{h}_{\sigma, j}= \begin{cases}\tilde{g}_{j} L^{\left(j \wedge j_{a b}\right)[\phi]} 2^{\left(j-j_{a b}\right)_{+}} & \mathfrak{h}=\ell \\
\tilde{g}_{j}^{1 / 4} L^{\left(j \wedge j_{a b}\right)[\phi]} 2^{\left(j-j_{a b}\right)} & \mathfrak{h}=h,\end{cases}
\end{gathered}
$$

where $[\phi]=\frac{d-2}{2}, x_{+}=\max \{x, 0\}$, and where the coalescence scale $j_{a b}$ is defined in (1.28).

\subsubsection{Norm for perturbative coordinate}

As a vector space, $\mathcal{Q}$ is isomorphic to $\mathbb{C}^{8}$ since a polynomial in $\mathcal{Q}$ is determined by eight coupling constants. Although all norms on $\mathbb{C}^{8}$ are equivalent, the coupling constants $\nu, \lambda^{a}, \lambda^{b}, q^{a}, q^{b}$ have natural scaling factors and we use a norm that takes this into account. We define a norm on $\mathcal{Q}$ by

$$
\|V\|_{\mathcal{Q}_{j}}=\max \left\{|g|,|z|,|y|, L^{2 j}|\nu|, \ell_{j} \ell_{\sigma, j}\left|\lambda^{a}\right|, \ell_{j} \ell_{\sigma, j}\left|\lambda^{b}\right|, \ell_{\sigma, j}^{2}\left|q^{a}\right|, \ell_{\sigma, j}^{2}\left|q^{b}\right|\right\} .
$$

The scaling in (1.33) reflects the fact that the coupling constants $g, z, y$ are associated to marginal field monomials (for $d=4$ ), whereas the $L^{2 j}$ reflects the fact that $\nu$ is associated to the relevant monomial $\tau$. The scaling of the observable coupling constants includes factors of $\ell_{j}$ or $\ell_{\sigma, j}$ for each boson or observable field, respectively, in the corresponding monomials in $V$.

Two useful subspaces of $\mathcal{Q}$ are the subspace $\mathcal{Q}^{(0)} \simeq \mathbb{C}^{5}$ consisting of elements of $\mathcal{Q}$ with $y=q^{a}=q^{b}=0$, and the subspace $\mathcal{Q}^{(1)} \simeq \mathbb{C}^{7}$ consisting of elements with $y=0$.

With [20, Lemma 3.1] and its proof, it follows that there is a $j$-independent constant $c>0$ such that

$$
c^{-1} \max _{B \in \mathcal{B}_{j}}\|V(B)\|_{T_{0, j}\left(\ell_{j}\right)} \leq\|V\|_{\mathcal{Q}_{j}} \leq c \max _{B \in \mathcal{B}_{j}}\|V(B)\|_{T_{0, j}\left(\ell_{j}\right)} .
$$

\subsubsection{Norms for non-perturbative coordinate}

Recall from [20, Section 1.1.6] the definition of the $T_{\phi, j}\left(\mathfrak{h}_{j}\right)$ seminorm. Recall also from [20, Definition 1.1, (1.38), (1.41)] the definition of the two norm pairs on $\mathcal{N}\left(X^{\square}\right)$ given, for $F \in \mathcal{N}\left(X^{\square}\right)$, by

$$
\begin{gathered}
\|F\|_{j}=\sup _{\phi \in \mathbb{C}^{\Lambda}} \frac{\|F\|_{T_{\phi, j}\left(\ell_{j}\right)}}{G_{j}(X, \phi)}, \quad\|F\|_{j+1}=\|F\|_{T_{0, j+1}\left(\ell_{j+1}\right)}, \\
\|F\|_{j}=\sup _{\phi \in \mathbb{C}^{\Lambda}} \frac{\|F\|_{T_{\phi, j}\left(h_{j}\right)}}{\tilde{G}_{j}(X, \phi)}, \quad\|F\|_{j+1}=\sup _{\phi \in \mathbb{C}^{\Lambda}} \frac{\|F\|_{T_{\phi, j+1}\left(h_{j+1}\right)}}{\tilde{G}_{j+1}^{\gamma}(X, \phi)},
\end{gathered}
$$

in terms of an arbitrary parameter $\gamma \in(0,1]$. To handle these norms simultaneously we will write them all as $\|F\|_{\mathcal{G}_{k}}$ with $k=j, j+1$. For the first pair. we write $\mathcal{G}_{j}=G_{j}\left(\ell_{j}\right)$ and $\mathcal{G}_{j+1}=T_{0, j+1}\left(\ell_{j+1}\right)$, 
and for the second pair we write $\mathcal{G}_{j}=\tilde{G}_{j}\left(h_{j}\right)$ and $\mathcal{G}_{j+1}=\tilde{G}_{j}^{\gamma}\left(h_{j+1}\right)$. Sometimes we omit parameters such as $j$ and $\mathfrak{h}_{j}$ when we think their values are clear from context. Note that the notation is potentially misleading because the dependence on the parameter $\mathfrak{h}_{k}$ refers to the $T_{\phi}$ part of this norm, not the regulators which are defined in [20, (1.38), (1.41)] always in terms of $\ell_{k}$.

In (1.35) we actually only have a $T_{0}$ semi-norm, not a norm. Let $\mathcal{I}(\mathbb{V})=\left\{F \in \mathcal{N}(\mathbb{V}) \mid\|F\|_{T_{0}}=\right.$ $0\}$. The set $\mathcal{I}(\mathbb{V})$ is an ideal in the algebra $\mathcal{N}$, since the $T_{0}$ semi-norm has the product property. Thus the $T_{0}$ semi-norm on $\mathcal{N}$ defines a norm on the quotient space $\mathcal{N} / \mathcal{I}$. We work in the quotient space, and thus regard $T_{0}$ as a norm rather than a semi-norm.

The above norms are defined on $\mathcal{N}(X)$, but to measure the size of elements of $\mathcal{K}$, which are maps $X \mapsto F(X)$ from polymers $X$ into $\mathcal{N}\left(X^{\square}\right)$, we include a weight for the size of $X$ as well. Thus we let $W: \mathcal{P}_{j} \times \mathbb{C}^{\mathbb{V}} \rightarrow(0, \infty)$ be a fixed strictly positive weight function. We say that $F \in \mathcal{K}_{j}$ vanishes at weighted infinity if for each $X \in \mathcal{P}_{j}$,

$$
\lim _{\|\phi\|_{\Phi_{j}(X)} \rightarrow \infty}\|F(X)\|_{T_{\phi}(\mathfrak{h})} W^{-1}(X, \phi)=0 .
$$

Let $\mathcal{F}_{j}(W)$ be the vector subspace of $\mathcal{K}_{j}$ consisting of elements $F$ which vanish at weighted infinity. We define a norm on $\mathcal{F}_{j}(W)$ by

$$
\|F\|_{\mathcal{F}_{j}(W)}=\sup _{X \in \mathcal{C}, \phi \in \mathbb{C}^{\mathbb{V}}}\|F(X)\|_{T_{\phi}(\mathfrak{h})} W^{-1}(X, \phi) .
$$

Now we make choices of $W=W_{j}$ that connect these norms to the two norm pairs (1.35)-(1.36). For $a>0$ and $X \in \mathcal{P}_{j}$, let

$$
f_{j}(a, X)=a\left(|X|_{j}-2^{d}\right)_{+} .
$$

Note that $f_{j}(a, X)=0$ for any small set $X$. For $\mathcal{G}_{j}$ a regulator, and given $\rho_{j} \in(0,1)$, let

$$
W(X, \phi)=\rho_{j}^{f_{j}(a, X)} \mathcal{G}_{j}(X, \phi)
$$

The factor $\rho_{j}^{f_{j}(a, X)}$ replaces the constant $A^{-1}$ used in many other papers in a version of (1.41), e.g., in [14, (6.10)]. Then for each of the four norms in the two norm pairs we have a choice of $W$ and scale $k=j, j+1$ such that

$$
\|F\|_{\mathcal{F}_{k}(W)}=\sup _{X \in \mathcal{C}_{k}} \rho_{k}^{-f_{k}(a, X)}\|F(X)\|_{k}
$$

with norms on the right-hand side as in (1.35) and (1.36). We denote the four normed spaces determined by (1.41) by $\mathcal{F}_{j}(G), \mathcal{F}_{j+1}\left(T_{0}\right)$ and $\mathcal{F}_{j}(\tilde{G}), \mathcal{F}_{j+1}\left(\tilde{G}^{\gamma}\right)$. The space $\mathcal{F}_{j+1}\left(T_{0}\right)$ is special, in that it has no dependence on $\phi$, and we have simply

$$
\|F\|_{\mathcal{F}_{j+1}\left(T_{0}\right)}=\sup _{X \in \mathcal{C}_{j+1}} \rho_{j+1}^{-f_{j+1}(a, X)}\|F(X)\|_{T_{0, j+1}(\mathfrak{h})} .
$$

The space $\mathcal{F}_{j+1}\left(T_{0}\right)$ is the set of elements of $\mathcal{K}_{j+1}$ for which the above norm is finite. We do have a norm here, rather than a semi-norm, because we have taken the quotient space that factors out elements of semi-norm zero, as discussed in Section 1.7. 
Fix $\Omega>1$ (a good choice is $\Omega=2$ ) and recall from [20, (1.69) $-(1.70)]$ the $\Omega$-scale $j_{\Omega}$ and the sequence $\chi_{j}=\Omega^{-\left(j-j_{\Omega}\right)_{+}}$. We make two choices of $\rho$, namely

$$
\rho_{j}=\bar{\epsilon}_{j}\left(\mathfrak{h}_{j}\right)= \begin{cases}\chi_{j}^{1 / 2} \tilde{g}_{j} & \mathfrak{h}_{j}=\ell_{j} \\ \chi_{j}^{1 / 2} \tilde{g}_{j}^{1 / 4} & \mathfrak{h}_{j}=h_{j},\end{cases}
$$

consistent with the definition of $\bar{\epsilon}_{j}$ in [20, (1.92)]. The $\mathfrak{h}=\ell$ choice of $\rho$ is used for $\mathcal{F}(G)$ and $\mathcal{F}\left(T_{0}\right)$, whereas the $\mathfrak{h}=h$ choice is used for $\mathcal{F}(\tilde{G})$ and $\mathcal{F}\left(\tilde{G}^{\gamma}\right)$. We set

$$
\omega_{j}=\frac{\bar{\epsilon}_{j}(\ell)}{\bar{\epsilon}_{j}(h)}=\tilde{g}_{j}^{3 / 4},
$$

and define another norm on $\mathcal{K}_{j}$ by

$$
\|K\|_{\mathcal{W}_{j}}=\max \left\{\|K\|_{\mathcal{F}_{j}(G)}, \omega_{j}^{3}\|K\|_{\mathcal{F}_{j}(\tilde{G})}\right\}
$$

By definition,

$$
\|K(X)\|_{T_{\phi, j}\left(\ell_{j}\right)} \leq\|K(X)\|_{G_{j}\left(\ell_{j}\right)} G_{j}(X, \phi) \leq\|K\|_{\mathcal{W}_{j}} G_{j}(X, \phi) \quad \text { for any } X \in \mathcal{S}_{j}
$$

On the right-hand side of (1.45), we choose $a \in\left(0, \frac{1}{4} 2^{-d}\right)$ as the value of $a$ in the exponent $f_{j}$ in the weight $\bar{\epsilon}_{j}$ appearing in the definitions of $\mathcal{F}_{j}(G)$ (we make the same choice for $\mathcal{F}_{j}\left(T_{0}\right)$ ), whereas we choose $\tilde{a}=4 a \in\left(0,2^{-d}\right)$ in the definition of $\mathcal{F}_{j}(\tilde{G})$. This particular choice produces the same power of $\tilde{g}$ for each of $\bar{\epsilon}(\ell)^{f_{j}(a, X)}$ and $\bar{\epsilon}(h)^{f_{j}(\tilde{a}, X)}$, and this plays a role in the proof of Lemma 2.4

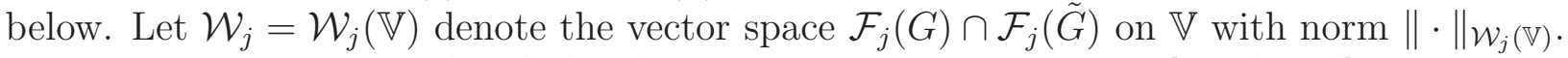

Each the four norms (1.35)-(1.36) obeys the product property [20, (1.44)], and our analysis relies heavily on this. The product property is spoiled in an unequally weighted maximum of two of these norms, due to the weight. For this reason, we do not have a version of the $\mathcal{W}$ norm obeying the product property, and consequently we often work directly with $\mathcal{F}$ norms instead. The following proposition is proved in Proposition A.3

Proposition 1.8. For either of the two choices $\mathbb{V}=\mathbb{Z}^{d}$ or $\mathbb{V}=\Lambda$, each of the spaces $\mathcal{F}(G), \mathcal{F}(\tilde{G})$, $\mathcal{F}\left(T_{0}\right)$ and $\mathcal{W}$ is a Banach space.

The $\mathcal{W}$ norm depends on the parameter $\tilde{g}_{j}$ appearing in (1.43), and also through the parameter $h_{j}=k_{0} L^{-j d / 4} \tilde{g}_{j}$ appearing in the norm of $\mathcal{F}_{j}(\tilde{G})$. In addition, it depends on $m^{2}$ since $\chi$ of (1.43) depends on $m^{2}$. The following lemma measures the effect on the norm under variation of these two parameters. The lemma is not used in the present paper but it is recorded here for use in [6].

Lemma 1.9. The norms $\mathcal{W}_{j}\left(m^{2}, \tilde{g}_{j}\right)$ and $\mathcal{W}_{j}\left(0, \tilde{g}_{j}\right)$ are identical when $j \leq j_{\Omega}$. In addition, if $\tilde{g}_{j}^{\prime} \leq \tilde{g}_{j}<1$ and $m^{\prime 2} \geq m^{2}>0$, then in the limit of small $\tilde{g}_{j}-\tilde{g}_{j}^{\prime}$, for all $K \in \mathcal{W}_{j}\left(m^{\prime 2}, \tilde{g}^{\prime}\right)$,

$$
\|K\|_{\mathcal{W}_{j}\left(m^{2}, \tilde{g}_{j}\right)} \leq\left(1+O\left(\tilde{g}_{j}-\tilde{g}_{j}^{\prime}\right)\right)\|K\|_{\mathcal{W}_{j}\left(m^{\prime 2}, \tilde{g}_{j}^{\prime}\right)}
$$

Proof. The first statement holds because $\chi_{j}\left(m^{2}\right)=\chi_{j}(0)=1$ when $j \leq j_{\Omega}$, by definition.

For the second statement, we first consider the dependence on $\mathrm{m}^{2}$. It follows from the definition of $\chi_{j}$ in [20, (1.69)-(1.70)] that $\chi_{j}$ is monotone non-increasing in $m^{2}$, and hence $1 / \chi_{j}$ is monotone non-decreasing in $m^{2}$. Consequently, increasing $m^{2}$ causes $1 / \rho_{j}$ to increase, consistent with (1.47). 
Next, we consider the $\tilde{g}$ dependence. The norm $\|K\|_{\mathcal{F}_{j}(G)}$ is monotone decreasing in $\tilde{g}_{j}$, by definition. By definition, $h_{j}=k_{0} L^{-j d / 4} \tilde{g}_{j}^{-1 / 4}$ is also monotone decreasing in $\tilde{g}_{j}$, so the norm $\|K\|_{\mathcal{F}_{j}(\tilde{G})}$ is also monotone decreasing in $\tilde{g}_{j}$ by definition. The factor $\omega_{j}^{3}$ in the $\mathcal{W}_{j}(\tilde{g})$ norm is however monotone increasing, but since it is continuous, the claim follows.

\subsubsection{Norm for scale $N$}

Special attention is required for the norm at scale $N$, but there is also increased flexibility. Our need to have both the $G$ and $\tilde{G}$ norms is explained in [20, Section 1.2.1], and it is connected with the need to propagate estimates from one scale to another. Once scale $N$ has been reached, there is no further propagation. In particular, it is not a problem if there is degradation of the $G$ regulator at the final scale $N$. We employ the $T_{0}$ and $\tilde{G}$ norms precisely to prevent such degradation from accumulating over an unbounded number of scales, but for a single scale it is permissible.

At scale $N$, the torus $\Lambda$ is the only polymer, and it is a single block. With the above in mind, for scale $N$ we define the $\mathcal{W}_{N}$ norm of $F: \mathcal{P}_{N} \rightarrow \mathcal{N}$ by

$$
\|F\|_{\mathcal{W}_{N}}=\sup _{\phi \in \mathbb{C}^{\Lambda}} \frac{\|F(\Lambda)\|_{T_{\phi, N}}}{G_{N}(\Lambda, \phi)^{10}} .
$$

The power "10" in the denominator reflects the regulator degradation mentioned above, and any fixed larger value could be used instead. (Cf. [20, Remark 1.4].)

\subsection{Main results}

In this section, we present our main results. Throughout we typically omit the subscript $j$ and abreviate the subscript $j+1$ to + . Thus we write $(V, K)$ rather than $\left(V_{j}, K_{j}\right)$, and write $\left(V_{+}, K_{+}\right)$ rather than $\left(V_{j+1}, K_{j+1}\right)$. We first state results for the finite volume renormalisation group map on a torus, and then describe the explicit construction of the map $(V, K) \mapsto V_{+}$. Following this, we extend the definition of the renormalisation group map to infinite volume, and state results for the infinite volume map. The infinite volume map is important in [6], to define a dynamical system that is not limited to flow through only a finite number of scales.

\subsubsection{Main result in finite volume}

To simplify the notation, we write $V=V_{j}, I=I_{j}(V), K=K_{j}$, and we wish to construct $\delta q_{+}=\delta q_{j+1}, V_{+}=V_{j+1}, I_{+}=I_{j+1}\left(V_{+}\right), K_{+}=K_{j+1}$ such that the action of $\mathbb{E}_{+} \theta=\mathbb{E}_{j+1} \theta$ is as stated in (1.18), i.e.,

$$
\mathbb{E}_{+} \theta(I(V) \circ K)(\Lambda)=e^{\delta q_{+} \sigma \bar{\sigma}}\left(I_{+}\left(V_{+}\right) \circ K_{+}\right)(\Lambda) .
$$

At (1.18), we defined the renormalisation group map $(V, K) \mapsto\left(\delta q_{+}, V_{+}, K_{+}\right)$, with $V, V_{+} \in \mathcal{Q}^{(0)} \simeq$ $\mathbb{C}^{5}$ and $\delta q_{+} \in \mathbb{C}$.

We define a mapping $V \mapsto V^{(0)}$ from $\mathcal{Q} \simeq \mathbb{C}^{8}$ to $\mathcal{Q}^{(0)} \simeq \mathbb{C}^{6}$, by replacing $z \tau_{\Delta}+y \tau_{\nabla \nabla}+q_{a b} \sigma \bar{\sigma}$ in $V \in \mathcal{Q}$ by $(z+y) \tau_{\Delta}$ in $V^{(0)} \in \mathcal{Q}^{(0)}$. Similarly, we define $V \mapsto V^{(1)}$ from $\mathcal{Q} \simeq \mathbb{C}^{8}$ to $\mathcal{Q}^{(1)} \simeq \mathbb{C}^{7}$ by replacing $z \tau_{\Delta}+y \tau_{\nabla \nabla}$ in $V \in \mathcal{Q}$ by $(z+y) \tau_{\Delta}$ in $V^{(1)} \in \mathcal{Q}^{(1)}$. Recall the map $V \mapsto V_{\mathrm{pt}}(V)$ from $\mathcal{Q}$ 
to $\mathcal{Q}$ defined in [7, (3.23)]. Given $V, V_{+} \in \mathcal{Q}^{(0)}$ and $\delta q_{+}^{a}, \delta q_{+}^{b} \in \mathbb{C}$, we define $R_{+} \in \mathcal{Q}^{(1)}$ and $\delta q_{+} \in \mathbb{C}$ by

$$
\left(V_{+}, \delta q_{+}^{a}, \delta q_{+}^{b}\right)=V_{\mathrm{pt}}^{(1)}(V)+R_{+}, \quad \delta q_{+}=\frac{1}{2}\left(\delta q_{+}^{a}+\delta q_{+}^{b}\right) .
$$

Conversely, given $V, R_{+},(1.50)$ determines $\left(V_{+}, \delta q_{+}^{a}, \delta q_{+}^{b}\right)$, and we state our results about the map $(V, K) \mapsto\left(R_{+}, K_{+}\right)$. This then uniquely specifies a map $(V, K) \mapsto\left(\delta q_{+}, V_{+}, K_{+}\right)$. The construction of $R_{+}$is explicit and relatively simple, and its formula is written in Section 1.8.2 below.

To state our estimates on $R_{+}$, we recall the definition of $\mathcal{S}$ from Definition 1.6, write $B_{\mathcal{Q}^{(0)}}(r)=$ $\left\{V \in \mathcal{Q}^{(0)}:\|V\|_{\mathcal{Q}}<r\right\}$, and define

$$
B_{T_{0}}(r)=\left\{K \in \mathcal{K}: \sup _{Y \in \mathcal{S}}\|K(Y)\|_{T_{0}(\ell)}<r\right\}
$$

Also, for $j<N$, the covariances $C_{j}$ are identified with those in the decomposition of the infinite volume covariance $\left(-\Delta_{\mathbb{Z}^{d}}+m^{2}\right)^{-1}$, and these are defined and obey the required estimates when $m^{2} \in[0, \delta]$ for small $\delta$. For $C_{N, N}$, we restrict to $m^{2} \in\left[\delta L^{-2(N-1)}, \delta\right]$ as discussed in Section 1.3 . Thus we define the intervals

$$
\mathbb{I}_{j}= \begin{cases}{[0, \delta]} & j<N \\ {\left[\delta L^{-2(N-1)}, \delta\right]} & j=N .\end{cases}
$$

We can now state our estimates on $R_{+}$. The analyticity statement concerns an analytic map from one complex Banach space to another. By definition, such a map is analytic on an open domain if it is continuously Fréchet differentiable on that domain (see, e.g., [35, Appendix A] or [22] for the elements of Banach space analyticity). In the derivative estimates, the $L^{p, q}$ norm is the norm of a multi-linear operator from $\mathcal{Q}^{p} \times \mathcal{K}_{j}^{q}$ to $\mathcal{Q}_{j+1}$. The continuity in $m^{2}$ is in the interval $[0, \delta]$ for all scales $j$; the restriction for $j=N$ occurs later.

The proof of Theorem 1.10 is given in Section 2.1.

Theorem 1.10. Let $\mathbb{V}=\Lambda$ and $j<N(\Lambda)$. There exists $r_{Q}>0$ (small) such that the map $R_{+}: B_{\mathcal{Q}^{(0)}}\left(r_{Q}\right) \times \mathcal{K} \times \mathbb{I}_{+} \rightarrow \mathcal{Q}_{+}$is analytic in $V$, quadratic in $K$, continuous in $m^{2} \in[0, \delta]$, and independent of $N$. There exists $M$ (large, dependent on $p, q \in \mathbb{N}_{0}$, independent of $r_{0}, r_{Q}$ ) such that for $r_{0} \in\left(0, r_{Q}\right)$ and $\left(V, K, m^{2}\right) \in B_{\mathcal{Q}^{(0)}}\left(r_{Q}\right) \times B_{T_{0}}\left(r_{0}\right) \times[0, \delta]$,

$$
\left\|D_{V}^{p} D_{K}^{q} R_{+}\right\|_{L^{p, q}} \leq M \begin{cases}r_{0} r_{Q}^{-p} & p \geq 0, q=0 \\ r_{Q}^{1-p-q} & p \geq 0, q=1,2 \\ 0 & p \geq 0, q \geq 3 .\end{cases}
$$

Each Fréchet derivative $D_{V}^{p} D_{K}^{q} R_{+}$, when applied as a multilinear map to directions $\dot{V}$ in $\left(\mathcal{Q}^{0}\right)^{p}$ and $\dot{K}$ in $\mathcal{K}^{q}$, is jointly continuous in all arguments, $m^{2}, V, K, \dot{V}, \dot{K}$. In particular, it is jointly continuous on the boundary $m^{2}=0$.

Next, we specify domains for the $K_{+}$part of the RG map. Let $j<N(\Lambda)$. We fix $\tilde{g}_{j}, \tilde{g}_{j+1}$ obeying (1.30). As in [20, (1.84)], we fix a universal constant $C_{\mathcal{D}}$ and for $x=\nu, z, \lambda^{a}, \lambda^{b}$ define $r_{x, j}$ by

$$
L^{2 j} r_{\nu, j}=r_{z, j}=C_{\mathcal{D}} \tilde{g}_{j}, \quad r_{\lambda, j}=C_{\mathcal{D}}
$$


We then define

$$
\begin{gathered}
\mathcal{D}_{j}=\left\{(g, \nu, z, \lambda) \in \mathbb{C}^{4}: C_{\mathcal{D}}^{-1} \tilde{g}_{j}<\operatorname{Re} g<C_{\mathcal{D}} \tilde{g}_{j},|\operatorname{Im} g|<\frac{1}{10} \operatorname{Re} g\right. \\
\left.|x| \leq r_{x} \text { for } x=\nu, z, \lambda^{a}, \lambda^{b}\right\}
\end{gathered}
$$

which is the important stability domain defined in [20, (1.83)] restricted to $y=q_{a b}=0$. The mass $m^{2}$ determines the sequence $\chi_{j}$ defined above (1.43) (in particular, $\chi_{j}=1$ for all $j$ when $m^{2}=0$ ). For $j<N$ and $R>0$ (large), we define domains $\mathbb{D}_{j}=\mathbb{D}_{j}(\mathbb{V}) \subset \mathcal{Q} \times \mathcal{K}_{j}(\mathbb{V})$ by

$$
\mathbb{D}_{j}(\mathbb{V})=\mathcal{D}_{j} \times B_{\mathcal{W}_{j}(\mathbb{V})}\left(R \chi_{j}^{3 / 2} \tilde{g}_{j}^{3}\right) .
$$

The radius $R \chi_{j}^{3 / 2} \tilde{g}_{j}^{3}$ of the ball in (1.56) depends on $m^{2}$ via $\chi_{j}$, and increases as $m^{2}$ decreases. By definition,

$$
B_{\mathcal{W}_{j}}\left(R \chi_{j}^{3 / 2} \tilde{g}_{j}^{3}\right) \subset B_{\mathcal{F}_{j}(G)}\left(R \chi_{j}^{3 / 2} \tilde{g}_{j}^{3}\right) \subset B_{T_{0}}\left(R \chi_{j}^{3 / 2} \tilde{g}_{j}^{3}\right),
$$

so with the choices $r_{Q}=C_{\mathcal{D}} \tilde{g}_{j}$ and $r_{0}=R \chi_{j}^{3 / 2} \tilde{g}_{j}^{3}$, the domain of Theorem 1.10 is larger than $\mathbb{D}_{j}$ :

$$
\mathbb{D}_{j} \subset B_{\mathcal{Q}^{(0)}}\left(C_{\mathcal{D}} \tilde{g}_{j}\right) \times B_{T_{0}}\left(R \chi_{j}^{3 / 2} \tilde{g}_{j}^{3}\right) .
$$

The following theorem, which constructs the $K_{+}$part of the renormalisation group map, is our main result. The construction of $K_{+}$is explicit, but it is not simple. The theorem is a local existence theorem for the dynamical system that RG generates: it says in particular that the map $(V, K) \mapsto K_{+}$is defined and contractive when $(V, K)$ is in the domain $\mathbb{D}_{j}$ (which in particular requires that $K$ be in a small ball). The contractivity appears in (1.60), due to $\kappa<1$. It is also evidenced by the fact that we can choose $R$ to be large without affecting the value of $M$, so in particular if we choose $R=2 M$ then we see from the $p=q=0$ case of (1.60) that the radius of the ball for $K_{+}$is half that of the ball for $K$ in the domain $\mathbb{D}_{j}$. In the derivative estimates, the $L^{p, q}$ norm is the norm of a multi-linear operator from $\mathcal{Q}^{p} \times \mathcal{K}_{j}^{q}$ to $\mathcal{K}_{j+1}$.

Theorem 1.11. Let $\mathbb{V}=\Lambda$ and $j<N(\Lambda)$. Fix any $a \in\left(0,2^{-d}\right), R>0, C_{\mathcal{D}}$ (both as large as desired), and let $L$ be sufficiently large (depending on $R$ ). Let $p, q \in \mathbb{N}_{0}$. There exist $\delta$ (depending on $R, L$ ), $M>0$ (depending on $p, q, L$ but not $R$ ) and $\kappa=O\left(L^{-1}\right)$ such that for all $\tilde{g} \in(0, \delta)$ and $m^{2} \in \mathbb{I}_{j+1}$, there exists a map

$$
K_{+}: \mathbb{D}_{j}(\Lambda) \rightarrow \mathcal{W}_{j+1}(\mathbb{V})
$$

such that (1.49) holds. The map $K_{+}$is analytic in $(V, K)$, and, pointwise in $(V, K)$, satisfies the estimates

$$
\left\|D_{V}^{p} D_{K}^{q} K_{+}\right\|_{L^{p, q}} \leq \begin{cases}\kappa & p=0, q=1 \\ M \chi_{+}^{3 / 2} \tilde{g}_{+}^{3-p} & p \geq 0 \\ M \tilde{g}_{+}^{-p}\left(\chi_{+}^{1 / 2} \tilde{g}_{+}^{10 / 4}\right)^{1-q} & p \geq 0, q \geq 1 .\end{cases}
$$

By Theorem 1.10 and (1.58), under the hypotheses of Theorem 1.11, we also have

$$
\left\|D_{V}^{p} D_{K}^{q} R_{+}\right\|_{L^{p, q}} \leq \begin{cases}M \chi^{3 / 2} \tilde{g}^{3-p} & p \geq 0, q=0 \\ M \tilde{g}^{1-p-q} & p \geq 0, q=1,2 \\ 0 & p \geq 0, q \geq 3 .\end{cases}
$$


Furthermore, by (1.30), we can replace $\chi$ and $\tilde{g}$ in (1.61) by $\chi_{+}$and $\tilde{g}_{+}$at the cost of increasing $M$ by a bounded multiple depending only on $\Omega$.

Our construction of $K_{+}$gives it a local dependence on $K$, as formulated in the next proposition.

Proposition 1.12. For $U \in \mathcal{P}_{j+1}(\Lambda)$, the value of $K_{+}(U)$ depends on $K$ only via the restriction $\left.K\right|_{U \square}$ of $K$ to polymers in $\mathcal{P}_{j}\left(U^{\square}\right)$.

To gain some insight into the meaning of the norm estimates, suppose that the $p=q=0$ estimate of (1.60) holds at the final scale $j+1=N$, i.e., $\left\|K_{N}\right\|_{\mathcal{W}_{N}} \leq M \chi_{N}^{3 / 2} \tilde{g}_{N}^{3 / 2}$. In [6], we use the $\theta$ which appears in (1.49) at all scales, but in [5] the simpler case in which $\theta$ is omitted at the final scale is sufficient. We consider here the simpler case, in which in (1.49) the final integration leaves no dependence on the fields. There is only one non-empty polymer at the final scale, namely $\Lambda$ itself. We denote the effect of setting the boson and fermion fields to zero by a superscript 0 . Then $K_{N}^{0}(\Lambda)$ is a complex scalar, and we write its direct sum decomposition, as in (1.2), as $K_{N}^{0}(\Lambda)=K_{N ; \varnothing}^{0}+\sigma K_{N ; \sigma}^{0}+\bar{\sigma} K_{N ; \bar{\sigma}}^{0}+\sigma \bar{\sigma} K_{N ; \sigma \bar{\sigma}}^{0}$. By (1.46) and the definition of the norm in [19, (1.61)],

$$
\left\|K_{N}\right\|_{\mathcal{W}_{N}} \geq\left\|K_{N}(\Lambda)\right\|_{T_{0, N}\left(\ell_{N}\right)}=\left|K_{N ; \varnothing}^{0}\right|+\ell_{\sigma, N}\left|K_{N ; \sigma}^{0}\right|+\ell_{\sigma, N}\left|K_{N ; \bar{\sigma}}^{0}\right|+\ell_{\sigma, N}^{2}\left|K_{N ; \sigma \bar{\sigma}}^{0}\right|
$$

where

$$
\ell_{\sigma, N}=2^{N-j_{a b}} L^{j_{a b}[\phi]} \tilde{g}_{N},
$$

by (1.32). We always assume that $N$ is larger than the coalescence scale $j_{a b}$, so that $a, b$ can be identified with points on the torus. Also, it follows from (1.28) that $L^{j_{a b}}$ is bounded above and below by multiples of $|a-b|$ (in particular, $|a-b| \geq \frac{1}{2} L^{j_{a b}}$ ). Thus we conclude that

$$
\left|K_{N ; \varnothing}^{0}\right| \leq M \chi_{N}^{3 / 2} \tilde{g}_{N}^{3} \quad \text { and } \quad\left|K_{N ; \sigma \bar{\sigma}}^{0}\right| \leq \frac{M^{\prime}}{4^{N-j_{a b}}} \frac{1}{|a-b|^{2[\phi]}} \chi_{N}^{3 / 2} \tilde{g}_{N}
$$

for some $M^{\prime}$. This is used in [5].

We also consider the continuity of $K_{+}$in the mass parameter $m^{2} \in I_{j+1}$. This issue is complicated by the fact that the radius of the ball in $\mathcal{K}_{j}$ in the domain $\mathbb{D}_{j}$ of $(1.56)$ depends on $\chi_{j}$, which itself depends on $m^{2}$. Similarly, the space $\mathcal{W}_{j}$ depends on $\rho_{j}$, which also depends on $\chi_{j}$ and hence on $m^{2}$. To disentangle the domain from the mass parameter we wish to vary, we fix $\tilde{m}^{2} \in I_{j+1}$ and define $\tilde{\chi}_{j}=\chi_{j}\left(\tilde{m}^{2}\right)$, and use this to define the domain and space. Thus we define the spaces $\tilde{\mathcal{W}}_{j}$ by replacing $\chi_{j}$ by $\tilde{\chi}_{j}$ in (1.43), and we define the domains

$$
\tilde{\mathbb{D}}_{j}(\mathbb{V})=\mathcal{D}_{j} \times B_{\tilde{\mathcal{W}}_{j}(\mathbb{V})}\left(r \tilde{\chi}_{j}^{3 / 2} \tilde{g}_{j}^{3}\right)
$$

By definition, $\tilde{\chi}_{j}$ increases as $\tilde{m}^{2}$ decreases. Consequently the domain $\tilde{\mathbb{D}}_{j}$ increases as $\tilde{m}^{2}$ decreases, and hence if $(V, K) \in \tilde{\mathbb{D}}_{j}\left(\tilde{m}^{2}\right)$ for a fixed value of $\tilde{m}^{2}$, then $(V, K) \in \tilde{\mathbb{D}}_{j}\left(\left(\tilde{m}^{\prime}\right)^{2}\right)$ for all $\tilde{m}^{\prime} \leq \tilde{m}$. We also define the intervals

$$
\tilde{\mathbb{I}}_{j}=\tilde{\mathbb{I}}_{j}\left(\tilde{m}^{2}\right)= \begin{cases}{\left[\frac{1}{2} \tilde{m}^{2}, 2 \tilde{m}^{2}\right] \cap \mathbb{I}_{j}} & \left(\tilde{m}^{2} \neq 0\right) \\ {\left[0, L^{-2(j-1)}\right] \cap \mathbb{I}_{j}} & \left(\tilde{m}^{2}=0\right) .\end{cases}
$$


Theorem 1.13. Let $\mathbb{V}=\Lambda$ and $j<N(\Lambda)$. Let $a, R, C_{\mathcal{D}}, L, \delta, M, \kappa$ be as in Theorem 1.11. Let $\tilde{m}^{2} \in \mathbb{I}_{j+1}$. The map $K_{+}$of Theorem 1.11 extends to a map

$$
K_{+}: \tilde{\mathbb{D}}_{j}(\Lambda) \times \tilde{\mathbb{I}}_{j+1}\left(\tilde{m}^{2}\right) \rightarrow \tilde{\mathcal{W}}_{j+1}(\Lambda),
$$

which is analytic in $(V, K)$, and obeys the estimates (1.60). For $j+1<N$, every Fréchet derivative $D_{V}^{p} D_{K}^{q} R_{+}$, when applied as a multilinear map to directions $\dot{V}$ in $\left(\mathcal{Q}^{0}\right)^{p}$ and $\dot{K}$ in $\mathcal{W}^{q}$, is jointly continuous in all arguments $m^{2}, V, K, \dot{V}, \dot{K}$. The domain of joint continuity includes the boundary $m^{2}=0$, provided $(V, K)$ is in the domain $\tilde{\mathbb{D}}_{j}(\Lambda) \times \tilde{\mathbb{I}}_{j+1}\left(\tilde{m}^{2}\right)$ defined with $\tilde{m}^{2}=0$.

Our main results all include the presence of observables, corresponding to the observable fields $\sigma, \bar{\sigma}$. However, our construction is triangular, in the sense that the bulk part of $\left(V_{+}, K_{+}\right)$, obtained by setting $\sigma=\bar{\sigma}=0$, is the same as if no observables were present in the original $(V, K)$, i.e.,

$$
\pi_{\varnothing} V_{+}(V, K)=V_{+}\left(\pi_{\varnothing} V, \pi_{\varnothing} K\right), \quad \pi_{\varnothing} K_{+}(V, K)=K_{+}\left(\pi_{\varnothing} V, \pi_{\varnothing} K\right) .
$$

The map $\pi_{\varnothing}: \mathcal{N} \rightarrow \mathcal{N}_{\varnothing}$ is linear and bounded in $T_{0}$ norm, and therefore it is continuous in the topology of this norm. Furthermore $\pi_{\varnothing}: \mathcal{N} \rightarrow \mathcal{N}_{\varnothing}$ is a homomorphism of algebras, because it is evaluation at $\sigma=\bar{\sigma}=0$. Therefore, for any polynomial $F(V, K)$ in $V$ and $K$, we have $\pi_{\varnothing} F(V, K)=F\left(\pi_{\varnothing} V, \pi_{\varnothing} K\right)$ and the same is true for $T_{0}$ limits of polynomials. The first equation in (1.68) then follows from the analyticity statement in Theorem 1.10, which implies that $R_{+}$is the limit in $T_{0}$ norm of truncations of its power series in $V, K$. To obtain the second equation in (1.68), we similarly use Theorem 1.11 to approximate $K_{+}(V, K)$ in $T_{0}$ norm by a polynomial in $V$ and $K$.

In the presence of observables, (1.68) is supplemented by the statement that, for $x=a$ or $x=b$,

$$
\begin{aligned}
& \text { if } \pi_{x} V=0 \text { and } \pi_{x} K(X)=0 \text { for all } X \in \mathcal{P} \text { then } \\
& \pi_{x} R_{+}=\pi_{a b} R_{+}=0 \text { and } \pi_{x} K_{+}(U)=\pi_{a b} K_{+}(U)=0 \text { for all } U \in \mathcal{P}_{+} .
\end{aligned}
$$

In addition, $\lambda_{+}^{a}$ is independent of each of $\lambda^{b}, \pi_{b} K$, and $\pi_{a b} K$, and the same is true with $a, b$ interchanged. The statement in (1.69) concerning $K_{+}$is proved in Theorem 2.2(v), and the statements about $R_{+}$and $\lambda_{+}$are proved in Proposition 1.14.

\subsubsection{Flow of coupling constants in finite volume}

In this section, we explicitly define the map $R_{+}$of Theorem 1.10. The proof that this map obeys the estimates of Theorem 1.10 is deferred to Section 2.1.

We define $R_{+}$in such a way that the relevant and marginal parts of $K$ become incorporated into $V_{+}$. The operator Loc defined in [19] is designed expressly for this purpose. More precisely, given $Y \subset X \subset \Lambda$, the operator $\operatorname{Loc}_{X, Y}: \mathcal{N}_{X} \rightarrow \mathcal{Q}(Y)$ is defined in [19, Definition 1.17], and we employ here the field dimensions specified in [7, Section 3.2]. The specific details of the definition of Loc do not play a role in the present paper, but properties of Loc are important.

The following three steps define $q_{+} \in \mathbb{C}$ and $V_{+} \in \mathcal{Q}^{(0)}$ as explicit functions of $V, K$.

1. For $\mathbb{V}=\Lambda$, given $(V, K)$ and $B \in \mathcal{B}(\Lambda)$, we define

$$
Q(B)=\sum_{Y \in \mathcal{S}(\Lambda): Y \supset B} \operatorname{Loc}_{Y, B} I^{-Y} K(Y),
$$


where $I=I(V)$ and the negative exponent denotes the reciprocal, namely $I^{-Y}=\frac{1}{I(V, Y)}=$ $\prod_{B \in \mathcal{B}(Y)} \frac{1}{I(V, B)}$. The fact that (1.70) defines an element $Q \in \mathcal{Q}$ is proved in Lemma F.2, This defines a map

$$
(V, K) \mapsto \hat{V}=V-Q \in \mathcal{Q} .
$$

2. We compose the map (1.71) with the quadratic function $V \mapsto V_{\mathrm{pt}}(V)$ (defined in [7, (3.23) $]$ ) to obtain the map

$$
(V, K) \mapsto V_{\mathrm{pt}}(\hat{V})=V_{\mathrm{pt}}(V-Q) .
$$

The map $V_{\mathrm{pt}}=V_{\mathrm{pt}, j+1}$ is independent of $N$; see [7, Proposition 4.1, Definition 4.2].

3. Finally, we set

$$
V_{+}=V_{\mathrm{pt}}^{(0)}(\hat{V}), \quad q_{+}=q+\frac{1}{\sigma \bar{\sigma}} \pi_{a b} V_{\mathrm{pt}}(\hat{V}),
$$

with the superscript (0) denoting the operation described under (1.49) (replacement of $z \tau_{\Delta}+$ $y \tau_{\nabla \nabla}+q \sigma \bar{\sigma}$ by $\left.(z+y) \tau_{\Delta}\right)$.

We then define $I_{+} \in \mathcal{B} \mathcal{K}_{j+1}(\Lambda)$ by

$$
I_{+}=I_{j+1}\left(V_{+}\right)
$$

The above definition of $\left(V_{+}, q_{+}\right)$determines $R_{+}: \mathcal{Q}^{(0)} \times \mathcal{K}_{j}(\Lambda) \rightarrow \mathcal{Q}^{(1)}$ by

$$
R_{+}(V, K)=V_{\mathrm{pt}}^{(1)}(\hat{V})-V_{\mathrm{pt}}^{(1)}(V) .
$$

By definition, $R_{+}$is a quadratic function of $K$; its dependence on $V$ is nontrivial due to the dependence in $Q$ of $I$ on $V$.

We now interpret more explicitly the meaning of the estimate (1.53) for the flow of coupling constants determined by Theorem 1.10. By (1.50) and (1.75) $, V_{+}, \delta q_{+}$are determined by $(V, K) \in$ $\mathbb{D}_{j}$ by

$$
\begin{aligned}
V_{+}(V, K) & =V_{\mathrm{pt}}^{(0)}(V)+\left(V_{\mathrm{pt}}^{(0)}(\hat{V})-V_{\mathrm{pt}}^{(0)}(V)\right), \\
\left(\frac{1}{2} \delta q_{+}^{a}, \frac{1}{2} \delta q_{+}^{b}\right) & =\frac{1}{\sigma \bar{\sigma}} \pi_{a b} V_{\mathrm{pt}}(V)+\frac{1}{\sigma \bar{\sigma}} \pi_{a b}\left(V_{\mathrm{pt}}(\hat{V})-V_{\mathrm{pt}}(V)\right), \\
\delta q_{+}(V, K) & =\frac{1}{2}\left(\delta q_{+}^{a}+\frac{1}{2} \delta q_{+}^{b}\right) .
\end{aligned}
$$

The first terms on the right-hand sides of (1.76) -(1.77) are independent of $K$ and constitute the pertubative flow discussed at length in [8]. The last terms on the right-hand sides of (1.76) -(1.77) do depend on $K$ and constitute the non-perturbative correction to the perturbative flow. We write these non-perturbative corrections to the coupling constants $\left(g_{+}, z_{+}, \nu_{+}, \lambda_{+}^{a}, \lambda_{+}^{b}, q_{+}^{a}, q_{+}^{b}\right)$ as $v_{x, j}$, with $x=g, z, \nu, \lambda^{a}, \lambda^{b}, q^{a}, q^{b}$. The following proposition gives estimates for these correction terms.

Proposition 1.14. Let $j<N,(V, K) \in \tilde{\mathbb{D}}_{j}$, and $m^{2} \in \mathbb{I}_{j+1}$. The bounds

$$
\begin{aligned}
& v_{g, j}=O\left(\chi_{j}^{3 / 2} \tilde{g}_{j}^{3}\right), \quad v_{z, j}=O\left(\chi_{j}^{3 / 2} \tilde{g}_{j}^{3}\right), \quad v_{\nu, j}=O\left(\chi_{j}^{3 / 2} L^{-2 j} \tilde{g}_{j}^{3}\right), \\
& v_{\lambda, j}=O\left(\chi_{j}^{3 / 2} \tilde{g}_{j}^{2}\right) \mathbb{1}_{j<j_{a b}} \quad v_{q, j}=\frac{1}{|a-b|^{2[\phi]}} O\left(\chi_{j}^{3 / 2} 4^{-\left(j-j_{a b}\right)} \tilde{g}_{j}\right) \mathbb{1}_{j \geq j_{a b}}
\end{aligned}
$$


hold with L-dependent constants, where $\lambda$ represents either of $\lambda^{a}, \lambda^{b}$ and similarly for $q$. For $x=a$ or $x=b$, if $\pi_{x} V=0$ and $\pi_{x} K(X)=0$ for all $X \in \mathcal{P}$ then $\pi_{x} R_{+}=\pi_{a b} R_{+}=0$. In addition, $\lambda_{+}^{a}$ is independent of each of $\lambda^{b}, \pi_{b} K$, and $\pi_{a b} K$, and the same is true with a,b interchanged. Finally, each $v_{j}$ is continuous in $m^{2} \in[0, \delta]$.

Proof. Recall the definition of the $\mathcal{Q}$ norm in (1.33) and the definition of $\ell, \ell_{\sigma}$ from (1.31)-(1.32). With these, (1.53) gives the estimates (1.79), where the indicator functions for $v_{\lambda, j}, v_{q, j}$ arise as follows.

The last term on the right-hand side of (1.77) determines $v_{q, j}$. To justify the indicator function in (1.79) we have to show that $v_{q, j}$ is zero for $j<j_{a b}$. By the definition of $j_{a b}$ the distance between $a$ and $b$ is at least $\frac{1}{2} L^{j_{a b}}$. A small set of scale $j$ has diameter at most $c L^{j}$ for some $c$ depending only on $d=4$. For $j<j_{a b}$, since $L$ is large no small set at scale $j$ can contain both points $a$ and $b$, so $\pi_{a b} Q=0$ and hence $\pi_{a b} \hat{V}=\pi_{a b} V$. Since $V_{\mathrm{pt}}(\hat{V})-V_{\mathrm{pt}}(V)$ is quadratic in $\hat{V}$ we must also consider $\sigma \bar{\sigma}$ cross terms. Cross terms between $\sigma \bar{\phi}_{a}$ and $\bar{\sigma} \phi_{b}$ are zero because $\mathbb{E}_{j+1} \bar{\phi}_{a} \phi_{b}=C_{j+1 ; a b}=0$ when $j<j_{a b}$ (see [7, Lemma 5.8]). Thus $v_{q, j}$ is zero for $j<j_{a b}$.

Let $j \geq j_{a b}$. We have to prove that $v_{\lambda, j}=0$. This holds if $\pi_{a} \hat{V}=\pi_{a} V$ for the $\hat{V}$ and $V$ in the second term of (1.76). By (1.70) this holds if $\sigma \bar{\phi}$ and $\bar{\sigma} \phi$ are not in the range of Loc at scale $j$. This is discussed in [7, Section 3.2, where it is explained that the parameters in Loc are indeed selected so that for $j \geq j_{a b}, \sigma \bar{\phi}$ and $\bar{\sigma} \phi$ are not in the range of Loc.

Suppose now that $\pi_{a} V=0$ and $\pi_{a} K(X)=0$ for all $X \in \mathcal{P}$. Then $\pi_{a} V_{\mathrm{pt}}(V)=0$ by the formula for $\lambda_{\mathrm{pt}}$ in [7, (3.34)], and $\pi_{a} \hat{V}=0$ by (1.70)-(1.71). From this it follows that as required, $\pi_{x} R_{+}=\pi_{a b} R_{+}=0$. A similar argument applies when $a$ is replaced by $b$.

To see that $\pi_{a} V_{+}$is independent of each of $\pi_{b} V, \pi_{b} K, \pi_{a b} K$, we argue as follows. Since the flow of $\lambda^{a}$ stops at the coalescence scale, we may assume that $j<j_{a b}$. Let $X \in \mathcal{S}_{j}$ be a small set that contains $a$. Then $X$ cannot also contain $b$, so by the field locality assumption in Definition 1.7 , $\pi_{b} K(X)=\pi_{a b} K(X)=0$, and hence $\hat{V}$ does not depend on $\pi_{b} K$ or $\pi_{a b} K$. We appeal again to the formula for $\lambda_{\mathrm{pt}}$ in [7, (3.34)] to conclude that $\pi_{a} V_{\mathrm{pt}}(\hat{V})$ does not depend on $\lambda^{b}$ either. A similar argument applies when $a$ is replaced by $b$.

The continuity in $m^{2}$ of $v_{x, j}$ holds because the coefficients of $V_{\mathrm{pt}}$ (given explicitly in [7, (3.30)(3.35)]) are continuous in $m^{2} \in[0, \delta]$ by [7, Proposition 4.4].

Finally, for use in [5], we make the following additional observation. Let $\nu^{+}=\nu+2 g C_{0,0}$. We claim that

$$
\left|\nu_{+}-\nu^{+}\right|=O\left(\chi_{j}^{3 / 2} L^{-2 j} \tilde{g}_{j}^{2}\right) .
$$

To see this, we apply (1.76) and (1.79) to obtain $\left|\nu_{+}-\nu_{\mathrm{pt}}\right|=O\left(\chi_{j}^{3 / 2} L^{-2 j} \tilde{g}_{j}^{3}\right)$, so it suffices to show that $\left|\nu_{\mathrm{pt}}-\nu^{+}\right|=O\left(\chi_{j}^{3 / 2} L^{-2 j} \tilde{g}_{j}^{2}\right)$. For the latter, we see from [7, (3.31)] that $\nu_{\mathrm{pt}}-\nu^{+}$is a sum of terms that are each quadratic in the bulk coupling constants, and the claim then follows using $\tilde{g}$ bounds on the coupling constants and [7, Lemma 6.2].

\subsubsection{Main result in infinite volume}

Theorem 1.13 concerns the renormalisation group map on a torus $\Lambda$. We now develop a framework which permits an extension of the map to the infinite volume $\mathbb{Z}^{d}$, and state results concerning this extension. The main result is Theorem 1.19. 
To begin, we fix a scale $j<\infty$, and now regard Theorem 1.13 as simultaneously a statement about every torus $\Lambda$ with $N(\Lambda)>j$. We write the $\Lambda$-dependent input to Theorem 1.13 as $K_{\Lambda}$, so we have a family $\left(K_{\Lambda}\right)$ for all $\Lambda$ with $N(\Lambda)>j$, with each $K_{\Lambda} \in \mathcal{K}_{j}(\Lambda)$. The output of Theorem 1.13 includes a family $\left(K_{+, \Lambda}\right)$, with each $K_{+, \Lambda} \in \mathcal{K}_{j+1}(\Lambda)$. We associate to an embedding of a torus into a larger torus a compatibility condition on the family $\left(K_{\Lambda}\right)$ that is preserved by the renormalisation group map, and use this compatibility to construct the renormalisation group map in infinite volume.

For a nonempty polymer $X \in \mathcal{P}_{*}(\mathbb{V})$ (with $\mathbb{V}$ either $\Lambda$ or $\mathbb{Z}^{d}$ ) and a torus $\Lambda^{\prime}$, we say that $\iota$ is a coordinate map from $X$ to $\Lambda^{\prime}$ if (i) $\iota: X \rightarrow \Lambda^{\prime}$ is an injective map that maps nearest neighbour points in $X$ to nearest-neighbour points in the image set $\iota X$, (ii) nearest-neighbour points in $\iota X$ are mapped by $\iota^{-1}$ to nearest-neighbour points in $X$, (iii) if $X$ contains a point $x$ where there is an observable then $\iota x$ is the location of the observable in $\Lambda^{\prime}$. When we write $\iota^{-1}$, we always understand it to be the inverse defined on the image $\iota X$.

Next, we define the maps on $\mathcal{N}$ induced by $\iota$. Let $X$ be a polymer in $\mathcal{P}_{k}(\mathbb{V})$ for some scale $k$ and let $\iota$ be a coordinate map from $X$ to $\Lambda^{\prime}$. For $\phi$ in $\mathbb{C}^{\Lambda^{\prime}}$ we define $\phi_{\iota}$ in $\mathbb{C}^{X}$ by $\left(\phi_{\iota}\right)_{x}=\phi_{\iota x}$, and similarly for the Grassmann generators, $\left(\psi_{\iota}\right)_{x}=\psi_{\iota x}$. To define the action of $\iota$ on $\mathcal{N}$, it suffices to define the action of $\iota$ separately on the summands $\mathcal{N}^{\varnothing}, \mathcal{N}^{a}, \mathcal{N}^{b}, \mathcal{N}^{a b}$ in (1.2). We define an algebra isomorphism $\iota: \mathcal{N}^{\varnothing}(X) \rightarrow \mathcal{N}^{\varnothing}(\iota X)$ (the same name $\iota$ is used also for this map), as follows. An element

$$
F=\sum_{y \in \vec{\Lambda}^{*}} \frac{1}{y !} F_{y} \psi^{y}
$$

of $\mathcal{N}^{\varnothing}(X)$ is defined in terms of coefficients $F_{y}$ which are functions of fields in $X$, i.e., $F_{y}: \mathbb{C}^{X} \rightarrow \mathbb{C}$. We define $\iota\left(F_{y}\right): \mathbb{C}^{\iota X} \rightarrow \mathbb{C}$ by $\iota\left(F_{y}\right)(\phi)=F_{y}\left(\phi_{\iota}\right)$ and then set

$$
\iota F=\sum_{y \in \vec{\Lambda}^{*}} \frac{1}{y !} \iota\left(F_{y}\right) \psi_{\iota}^{y} .
$$

According to the definition of $\psi_{\iota}$ the product $\psi_{\iota}^{y}$ is a product of generators attached to points in $\iota X$, as it should be. The correspondence between $\iota$ as a coordinate map and $\iota$ as an algebra isomorphism is functorial: if $j=\iota \circ \iota^{\prime}$ as coordinate maps then $j=\iota \circ \iota^{\prime}$ as maps on $\mathcal{N}$. To define the action of $\iota$ on $\mathcal{N}^{a}(X)$, recall that the elements of $\mathcal{N}^{a}(X)$ have the form $\sigma F$ with $F \in \mathcal{N}^{\varnothing}(X)$. Then we set $\iota \sigma F=\sigma \iota F$. Thus $\iota$ does nothing to the observable fields $\sigma$ and $\bar{\sigma}$, which makes it clear how the action of $\iota$ on $\mathcal{N}^{b}, \mathcal{N}^{a b}$ is defined.

Any polymer $X$ on a torus $\Lambda$ whose diameter is less than that of $\Lambda$ will have a coordinate map to any larger torus $\Lambda^{\prime}$ (meaning $N\left(\Lambda^{\prime}\right) \geq N(\Lambda)$ ), and we say that $X$ is a coordinate patch on $\Lambda$ if $\operatorname{diam}(X) \leq \frac{1}{2} \operatorname{diam}(\Lambda)$. In particular, coordinate patches cannot wrap around the torus. We always assume that $L>2^{d}$, so that for scales $j<N(\Lambda)$ small sets are coordinate patches. The next definition introduces the compatibility condition mentioned above. It is called Property $\left(\mathbb{Z}^{d}\right)$ and it relates $K_{\Lambda}$ to $K_{\Lambda^{\prime}}$. Notice that the definition allows $\Lambda^{\prime}=\Lambda$. In this case Property $\left(\mathbb{Z}^{d}\right)$ is equivalent to the Euclidean invariance statement in Definition 1.7

Definition 1.15. We say that a family $\left(K_{\Lambda}\right)$ with each $K_{\Lambda} \in \mathcal{K}_{j}(\Lambda)$ has Property $\left(\mathbb{Z}^{d}\right)$ if

$$
\begin{gathered}
\iota K_{\Lambda}(X)=K_{\Lambda^{\prime}}(\iota X) \text { for all coordinate patches } X \in \mathcal{P}_{j}(\Lambda), \text { all } \Lambda^{\prime} \text { larger than } \Lambda, \\
\text { and all coordinate maps } \iota: X^{\square} \rightarrow \Lambda^{\prime} .
\end{gathered}
$$


Given a family $\left(K_{\Lambda}\right)$ that has Property $\left(\mathbb{Z}^{d}\right)$ we define $K_{\mathbb{Z}^{d}} \in \mathcal{K}_{j}\left(\mathbb{Z}^{d}\right)$ by

$$
K_{\mathbb{Z}^{d}}(X)=\iota^{-1}\left(K_{\Lambda}(\iota X)\right) \quad\left(X \in \mathcal{P}_{j}\left(\mathbb{Z}^{d}\right)\right)
$$

for some choice of $\Lambda$ with $\operatorname{diam}(\Lambda) \geq 2 \operatorname{diam}(X)$, and some choice of a coordinate map $\iota: X \rightarrow \Lambda$.

We claim that if $K$ has Property $\left(\mathbb{Z}^{d}\right)$ then $K_{\mathbb{Z}^{d}}(X)$ does not depend on $\iota$ or $\Lambda$. To see this, suppose we have two coordinate maps $\iota_{1}, \iota_{2}$ from $X$ to tori $\Lambda$ and $\Lambda^{\prime}$, with $\Lambda^{\prime}$ the larger torus. Then there exists a coordinate map $\iota_{3}$ from $\left(\iota_{1} X\right)^{\square}$ to $\Lambda^{\prime}$ such that $\iota_{2}=\iota_{3} \circ \iota_{1}$ on $X^{\square}$. Property $\left(\mathbb{Z}^{d}\right)$ implies that

$$
\iota_{3} K_{\Lambda}\left(\iota_{1} X\right)=K_{\Lambda^{\prime}}\left(\iota_{3} \circ \iota_{1} X\right)=K_{\Lambda^{\prime}}\left(\iota_{2} X\right)
$$

and the claim then follows by applying $\iota_{2}^{-1}=\iota_{1}^{-1} \circ \iota_{3}^{-1}$ to both sides.

For a function $F$ defined on polymers in $\mathcal{P}_{j}(\mathbb{V})$ and a polymer $Y$ in $\mathcal{P}_{j}(\mathbb{V})$, let $\left.F\right|_{Y}$ denote the restriction of $F$ to $\mathcal{P}_{j}(Y)$, i.e. to scale- $j$ polymers $X \subset Y$. According to Proposition $1.12, K_{+, \Lambda}(U)$ depends on $K_{\Lambda}$ only via $\left.K_{\Lambda}\right|_{U}$, and for fixed $V$ we can therefore regard the map $K_{\Lambda} \rightarrow K_{+, \Lambda}$ defined by Theorem 1.13 as a family of maps $g_{\Lambda}:\left.K_{\Lambda}\right|_{U} \mapsto K_{+, \Lambda}(U)$ indexed by $\Lambda$.

We will prove the following proposition (see Theorem 2.2 (iii)).

Proposition 1.16. Let $U \in \mathcal{P}_{j+1}(\Lambda)$ be a coordinate patch and let $\iota: U^{\square} \rightarrow \Lambda^{\prime}$ with $\Lambda^{\prime}$ larger than $\Lambda$. Then $\iota g_{\Lambda}\left(\left.K_{\Lambda}\right|_{U^{\square}}\right)=g_{\Lambda^{\prime}}\left(\left.\iota K_{\Lambda}\right|_{U^{\square}}\right)$.

The following proposition shows that Property $\left(\mathbb{Z}^{d}\right)$ is preserved by the renormalisation group map.

Proposition 1.17. If the collection $\left(K_{\Lambda}\right)$ has Property $\left(\mathbb{Z}^{d}\right)$ then $\left(K_{+, \Lambda}\right)$ produced by Theorem 1.13 also has Property $\left(\mathbb{Z}^{d}\right)$.

Proof. Let $U \in \mathcal{P}_{j+1}(\Lambda)$ be a coordinate patch and let $\iota: U^{\square} \rightarrow \Lambda^{\prime}$ with $\Lambda^{\prime}$ larger than $\Lambda$. Then

$$
\iota K_{+, \Lambda}(U)=\iota g_{\Lambda}\left(\left.K_{\Lambda}\right|_{U^{\square}}\right)=g_{\Lambda^{\prime}}\left(\iota\left(\left.K_{\Lambda}\right|_{U^{\square}}\right)\right)=g_{\Lambda^{\prime}}\left(\left.K_{\Lambda^{\prime}}\right|_{(\iota U)^{\square}}\right)=K_{+, \Lambda^{\prime}}(\iota U),
$$

by Proposition 1.16 for the second equality, and by Property $\left(\mathbb{Z}^{d}\right)$ of $\left(K_{\Lambda}\right)$ for the third.

Now we define the infinite volume map $\left(V, K_{\mathbb{Z}^{d}}\right) \mapsto K_{+, \mathbb{Z}^{d}}$. We fix $V$ and drop it from the notation. Let $K_{\mathbb{Z}^{d}} \in \mathcal{K}\left(\mathbb{Z}^{d}\right)$ and $U \in \mathcal{P}_{j+1}\left(\mathbb{Z}^{d}\right)$. We choose a torus $\Lambda$ with $N(\Lambda)>j+1$ and a coordinate map $\iota: U^{\square} \rightarrow \Lambda$. We first aim to apply Lemma E.6 to define $K_{\Lambda} \in \mathcal{K}_{j}(\Lambda)$ appropriately associated to $K_{\mathbb{Z}^{d}}$. For this, let $\mathcal{X}=\iota^{\mathcal{C}} \mathcal{C}_{j+1}\left(U^{\square}\right)$, which is a class of subsets of $\Lambda$. Define $F: \mathcal{X} \rightarrow \mathcal{N}$ by $F=\iota \circ K_{\mathbb{Z}^{d}} \circ \iota^{-1}$. For a Euclidean automorphism $E$ of $\Lambda$, and for $X \in \mathcal{X}$ such that $E X \in \mathcal{X}$, there is an automorphism $E^{\prime}$ of $\mathbb{Z}^{d}$ such that $E^{\prime}\left(\iota^{-1} X\right)=\iota^{-1}(E X)$. It follows from the Euclidean covariance of $K_{\mathbb{Z}^{d}}$ that $K_{\mathbb{Z}^{d}} \circ E^{\prime}=E^{\prime} \circ K_{\mathbb{Z}^{d}}$, and it is then straightforward to check that $F(E X)=E(F(X))$, which is the main hypothesis for Lemma E.6. The hypothesis involving $W$ can be vacuously satisfied by choosing $W=\infty$, and the other hypotheses hold because $K_{\mathbb{Z}^{d}} \in \mathcal{K}_{j}\left(\mathbb{Z}^{d}\right)$. Therefore, by Lemma E.6, there exists an extension $\hat{F} \in \mathcal{K}_{j}(\Lambda)$ of $F$ such that $K_{\Lambda}$ defined by $K_{\Lambda}=\hat{F}$ satisfies

$$
\left.K_{\Lambda}\right|_{\iota U^{\square}}=\left.\iota \circ K_{\mathbb{Z}^{d}} \circ \iota^{-1}\right|_{\iota U^{\square}} .
$$


We then define $K_{+, \mathbb{Z}^{d}}(U) \in \mathcal{N}\left(U^{\square}\right)$ by

$$
K_{+, \mathbb{Z}^{d}}(U)=\iota^{-1} \circ g_{\Lambda}\left(\left.K_{\Lambda}\right|_{\iota U^{\square}}\right),
$$

and we must prove that this definition assigns the same value regardless of how we choose $\Lambda$ and $\iota$.

Let $\iota^{\prime}$ be another coordinate map from $U^{\square}$ into another torus $\Lambda^{\prime}$ with $N\left(\Lambda^{\prime}\right)>j+1$, and let

$$
\left.K_{\Lambda^{\prime}}\right|_{\iota^{\prime} U \square}=\left.\iota^{\prime} \circ K_{\mathbb{Z}^{d}} \circ \iota^{\prime-1}\right|_{\iota^{\prime} U} \text {. }
$$

Let $j=\iota^{\prime} \circ \iota^{-1}$. Then $j$ is a coordinate map from $\iota U^{\square} \subset \Lambda$ into $\Lambda^{\prime}$. By (1.88) and Proposition 1.16,

$$
\begin{aligned}
K_{+, \mathbb{Z}^{d}}(U) & =\left(\iota^{-1} \circ g_{\Lambda}\left(\left.K_{\Lambda}\right|_{\iota U^{\square}}\right)=\iota^{\prime-1} \circ j \circ g_{\Lambda}\left(\left.K_{\Lambda}\right|_{\iota U^{\square}}\right)\right. \\
& =\iota^{\prime-1} \circ g_{\Lambda}^{\prime}\left(\left.j K_{\Lambda}\right|_{\iota U^{\square}}\right)=\iota^{\prime-1} \circ g_{\Lambda}^{\prime}\left(\left.K_{\Lambda^{\prime}}\right|_{\iota^{\prime} U^{\square}}\right) .
\end{aligned}
$$

Therefore the definition of $K_{+, \mathbb{Z}^{d}}(U)$ does not depend on the choices in the definition. Furthermore, this defines a map $K_{\mathbb{Z}^{d}} \mapsto K_{+, \mathbb{Z}^{d}}$. Because the finite volume map preserves the symmetries of Definition 1.7 by Theorem 1.11, the infinite volume map also preserves these symmetries. The infinite volume map $K_{\mathbb{Z}^{d}} \mapsto K_{+, \mathbb{Z}^{d}}$ is the unlabelled arrow in the commutative diagram:

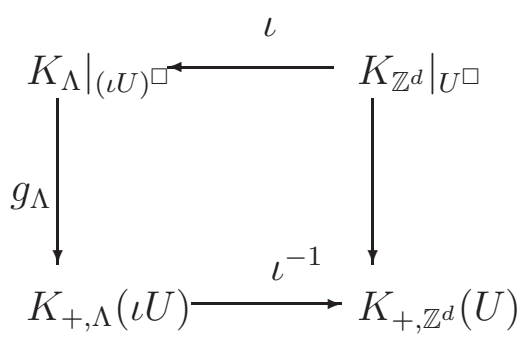

The map $R_{+}$of Section 1.8 .2 depends on $\Lambda$ because it is a function of $K \in \mathcal{K}(\Lambda)$. We now make this dependence explicit and write $R_{+, \Lambda}$ and $V_{+, \Lambda}$ in place of $R_{+}$and $V_{+}$. To complete the definition of the renormalisation group map in infinite volume, we define the infinite volume map $\left(V, K_{\mathbb{Z}^{d}}\right) \mapsto R_{+, \mathbb{Z}^{d}}$. This is similar to the construction of the map $K_{+, \mathbb{Z}^{d}}$, except $R_{+, \Lambda}$ has values in $\mathcal{Q}$ as opposed to values in $\mathcal{K}$.

To distinguish between scale- $(j+1)$ blocks in $\mathbb{Z}^{d}$ and blocks in a torus, we write $B$ for the former and $C$ for the latter. In particular, $R_{+, \Lambda}(C)$ (as in (1.10)) is an element of $\mathcal{N}(C)$. By (1.75), $R_{+, \Lambda}$ is defined in terms of $V_{\mathrm{pt}}$ and in terms of $Q$ of (1.70). By definition, $V_{\mathrm{pt}}$ evaluated on a block $C$ depends only on fields and their derivatives on $C$, and hence depends on the values of fields in a cube obtained by extending $C$ by a few vertices in each direction. The same is true for $Q$ on a scale- $j$ block. Together, these facts much more than imply that $R_{+, \Lambda}(C)$ depends only on the restriction of $K_{\Lambda}$ to polymers in $\mathcal{P}\left(C^{\square}\right)$.

Let $K_{\mathbb{Z}^{d}} \in \mathcal{K}\left(\mathbb{Z}^{d}\right)$ and $B \in \mathcal{B}_{+}\left(\mathbb{Z}^{d}\right)$. We choose a torus $\Lambda$ with $N(\Lambda)>j+1$ and a coordinate map $\iota: B^{\square} \rightarrow \Lambda$. As in the definition of $K_{\mathbb{Z}^{d}}$, choose $K_{\Lambda} \in \mathcal{K}(\Lambda)$ such that

$$
\left.K_{\Lambda}\right|_{\iota B^{\square}}=\left.\iota \circ K_{\mathbb{Z}^{d}} \circ \iota^{-1}\right|_{\iota B^{\square}} .
$$

We define $R_{+, \mathbb{Z}^{d}}(B) \in \mathcal{N}(B)$ by

$$
R_{+, \mathbb{Z}^{d}}(B)=\iota^{-1}\left(R_{+, \Lambda}\left(V, K_{\Lambda}\right)(\iota B)\right) .
$$


The values of $\left(R_{+, \mathbb{Z}^{d}}(B), B \in \mathcal{B}_{+}\left(\mathbb{Z}^{d}\right)\right)$ determine a unique element $R_{+, \mathbb{Z}^{d}} \in \mathcal{Q}$ and therefore we have a map $\left(V, K_{\mathbb{Z}^{d}}\right) \mapsto \mathcal{Q}$. The following proposition shows that this map does not depend on the choices of $\Lambda$ and $\iota$ made in its definition.

Proposition 1.18. (i) Let $\left(K_{\Lambda}\right)$ be a family that has property $\left(\mathbb{Z}^{d}\right)$. Then for any tori $\Lambda$ and $\Lambda^{\prime}$ with $N(\Lambda), N\left(\Lambda^{\prime}\right) \geq j+1$,

$$
R_{+, \Lambda}\left(V, K_{\Lambda}\right)=R_{+, \Lambda^{\prime}}\left(V, K_{\Lambda^{\prime}}\right) .
$$

(ii) The definition of $R_{+, \mathbb{Z}^{d}}(B)$ in (1.92) does not depend on the choice of torus $\Lambda$ or coordinate map $\iota$.

Proof. The proofs of (i) and (ii) require the following preparation. We fix $V$ and drop it from the notation, and let $\Lambda, \Lambda^{\prime}$ be as in part (i) of the proposition. Let $C \in \mathcal{B}(\Lambda)$ and let $j: C^{\square} \rightarrow \Lambda^{\prime}$ be a coordinate map. We use subscripts $\Lambda$ and $\Lambda^{\prime}$ to indicate membership in $\mathcal{N}(\Lambda)$ or $\mathcal{N}\left(\Lambda^{\prime}\right)$. Let $K_{\Lambda} \in \mathcal{K}(\Lambda)$ and $K_{\Lambda^{\prime}} \in \mathcal{K}\left(\Lambda^{\prime}\right)$ be any elements that satisfy

$$
j K_{\Lambda}(X)=K_{\Lambda^{\prime}}(j X) \quad \text { for } X \in \mathcal{P}\left(C^{\square}\right) .
$$

Recall the definition (1.70) of $Q$, which here we write as $Q_{\Lambda}$ since it depends on $K_{\Lambda}$. We claim that

$$
j\left(Q_{\Lambda}(C)\right)=Q_{\Lambda^{\prime}}(j C)
$$

By definition,

$$
j\left(Q_{\Lambda}(C)\right)=\sum_{Y \in \mathcal{S}(\Lambda): Y \supset C} j\left(\operatorname{Loc}_{Y, C} I_{\Lambda}^{-Y} K_{\Lambda}(Y)\right),
$$

and by a small variation of [19, Proposition 1.9] followed by (1.94),

$$
j\left(\operatorname{Loc}_{Y, C} I_{\Lambda}^{-Y} K_{\Lambda}(Y)\right)=\operatorname{Loc}_{j Y, j C} j\left(I_{\Lambda}^{-Y}\right) j\left(K_{\Lambda}(Y)\right)=\operatorname{Loc}_{j Y, j C} I_{\Lambda^{\prime}}^{-j Y} K_{\Lambda^{\prime}}(j Y) .
$$

Therefore

$$
j\left(Q_{\Lambda}(C)\right)=\sum_{Y^{\prime} \in \mathcal{S}\left(\Lambda^{\prime}\right): Y^{\prime} \supset j C} \operatorname{Loc}_{Y^{\prime}, j C} I_{\Lambda^{\prime}}^{-Y^{\prime}} K_{\Lambda^{\prime}}\left(Y^{\prime}\right)=Q_{\Lambda^{\prime}}(j C) .
$$

(i) Since $\left(K_{\Lambda}\right)$ has the $\left(\mathbb{Z}^{d}\right)$ property, (1.98) holds for all blocks $C \in \mathcal{B}(\Lambda)$. Therefore, $Q_{\Lambda^{\prime}}=Q_{\Lambda}$ as elements of $\mathcal{Q}$. By the $\Lambda$-independence of the map $V \mapsto V_{\mathrm{pt}}$ constructed in [7, Section 4.1] and the definition of $R_{+}$in (1.75) , it follows that $R_{+, \Lambda}\left(V, K_{\Lambda}\right)=R_{+, \Lambda^{\prime}}\left(V, K_{\Lambda^{\prime}}\right)$. This concludes the proof of (i).

(ii) Given $K_{\mathbb{Z}^{d}} \in \mathcal{K}\left(\mathbb{Z}^{d}\right)$, choose $\Lambda, \iota, K_{\Lambda}$ and $\Lambda^{\prime}, \iota^{\prime}, K_{\Lambda^{\prime}}$ so that (1.91) holds for both choices. In this case, recall that $N(\Lambda)$ and $N\left(\Lambda^{\prime}\right)$ are greater than $j+1$, and $\iota, \iota^{\prime}$ are defined on $B$ in $\mathcal{B}_{+}\left(\mathbb{Z}^{d}\right)$. Then (1.94) holds with $j=\iota^{\prime} \circ \iota^{-1}$ and $C=\iota B$. Therefore, by part (i),

$$
\begin{gathered}
\iota^{-1}\left(R_{+, \Lambda}\left(K_{\Lambda}\right)(\iota B)\right)=\iota^{\prime-1} j\left(R_{+, \Lambda}\left(K_{\Lambda}\right)(\iota B)\right)=\iota^{\prime-1}\left(R_{+, \Lambda}\left(K_{\Lambda}\right)(j \iota B)\right) \\
=\iota^{\prime-1}\left(R_{+, \Lambda^{\prime}}\left(K_{\Lambda^{\prime}}\right)(j \iota B)\right)=\iota^{\prime-1}\left(R_{+, \Lambda^{\prime}}\left(K_{\Lambda^{\prime}}\right)\left(\iota^{\prime} B\right)\right) .
\end{gathered}
$$

This shows that the definition of $R_{+, \mathbb{Z}^{d}}(B)$ in (1.92) does not depend on the choice of $\Lambda$ or $\iota$, and completes the proof. 
By combining (1.88) and Proposition 1.18, we obtain the infinite volume map

$$
\left(V, K_{\mathbb{Z}^{d}}\right) \mapsto\left(R_{+, \mathbb{Z}^{d}}, K_{+, \mathbb{Z}^{d}}\right)
$$

for all scales $j<\infty$. In contrast to the finite volume case, it is not a defining feature of this map that it preserves a circle product under expectation. Indeed we do not have an interpretation of the expectation nor of the circle product in infinite volume. What the infinite volume map does achieve is a simultaneous encoding of the restriction of the finite volume map to coordinate patches for all volumes (this effectively ignores the part of the finite volume map that concerns polymers that wrap around the torus). As such, we regard the infinite volume map as an inductive limit of the finite volume maps, which given a polymer $U$ captures the behaviour of $K_{+, \Lambda}(U)$ for all volumes $\Lambda$ whose diameter is at least twice that of $U$. The following theorem is an analogue of Theorems 1.10 and 1.13 (the former specialised as in (1.61)) that summarises the properties of the infinite volume map. It follows directly from Theorems 1.10 and 1.13 and the definition of the infinite volume map.

Theorem 1.19. Let $\mathbb{V}=\mathbb{Z}^{d}$ and $j<\infty$. In (1.36), set $\gamma=1$. Let $a, R, C_{\mathcal{D}}, L, \delta, M, \kappa$ be as in Theorem 1.11. Let $\tilde{m}^{2} \in I_{j+1}$. Then (1.100) defines maps

$$
R_{+, \mathbb{Z}^{d}}: \tilde{\mathbb{D}}_{j}\left(\mathbb{Z}^{d}\right) \times \tilde{\mathbb{I}}_{j+1}\left(\tilde{m}^{2}\right) \rightarrow \mathcal{Q}_{j+1}^{(1)}, \quad K_{+, \mathbb{Z}^{d}}: \tilde{\mathbb{D}}_{j}\left(\mathbb{Z}^{d}\right) \times \tilde{\mathbb{I}}_{j+1}\left(\tilde{m}^{2}\right) \rightarrow \tilde{\mathcal{W}}_{j+1}\left(\mathbb{Z}^{d}\right),
$$

which are analytic in $(V, K)$, and obey the estimates (1.60)-(1.61) with all the norms on $\mathbb{Z}^{d}$ (rather than on $\Lambda)$. Every Fréchet derivative $D_{V}^{p} D_{K}^{q} R_{+, \mathbb{Z}^{d}}$, when applied as a multilinear map to directions $\dot{V}$ in $\left(\mathcal{Q}^{0}\right)^{p}$ and $\dot{K}$ in $\mathcal{W}^{q}$, is jointly continuous in all arguments $m^{2}, V, K, \dot{V}, \dot{K}$. The domain of joint continuity includes the boundary $m^{2}=0$, provided $(V, K)$ is in the domain $\tilde{\mathbb{D}}_{j}\left(\mathbb{Z}^{d}\right) \times \tilde{\mathbb{I}}_{j+1}\left(\tilde{m}^{2}\right)$ defined with $\tilde{m}^{2}=0$.

\subsubsection{Generalisations of main results}

We have formulated our results in the specific setting of the supersymmetric representation of the 4-dimensional weakly self-avoiding walk model, defined by the polynomial $V$ given in terms of bulk and observable terms in (1.8). However, the results are flexible, and can be extended with little effort in (at least) the following two directions.

Other observables. The first extension is to consider a different choice of observables. The observable terms in (1.8) are suitable for the analysis of the two-point function in [5]. The choice of $\mathfrak{h}_{\sigma}$ made in (1.32) for the observable field $\sigma$ is designed to be as large as possible so that the observable terms in $V$ remain benign for the stability estimates of [20, and in particular for [20, Proposition 1.5]. Different choices of observables could be made with corresponding different choices of $\mathfrak{h}_{\sigma}$; what needs to be checked is that: (i) the stability estimates continue to hold with the new observables, which requires that $\mathfrak{h}_{\sigma}$ be not too large, and (ii) the analogue of the second estimate of (1.64) applies in the new setting, which requires that $\mathfrak{h}_{\sigma}$ be not too small. A specific example where both of these objectives can be met for other observables is given in [39], where watermelon networks for the 4-dimensional weakly self-avoiding walk are analysed at the critical point. These are networks of $p$ weakly mutually- and self-avoiding walks from the origin to a distant point $x$, and we study the asymptotic behaviour as $|x| \rightarrow \infty$, for $p>1$. 
The $|\varphi|^{4}$ model. The second extension concerns the 4-dimensional $n$-component $|\varphi|^{4}$ spin model, at weak coupling. In [9], we apply the results of the present paper to analyse the critical behaviour, in particular of the susceptibility. A simplification is that the $|\varphi|^{4}$ model is purely bosonicthere is no fermion field. A small complication is that the model is $O(n)$-symmetric rather than supersymmetric. Consequently, $V_{+}$acquires a constant term $\delta u$ from $V_{\mathrm{pt}}$, in a similar manner to the occurrence of $\delta q \sigma \bar{\sigma}$ in (1.49). The constant term is a bulk rather than an observable term, and consequently it occurs in $V(X)$ as $\delta u|X|_{0}$, where $|X|_{0}$ is the cardinality of $X$. In [9], we extract the constant term from the circle product, just as we do for $\delta q \sigma \bar{\sigma}$. This requires a small adaptation to the proof of Lemma 6.2. discussed in Remark 6.3. The inclusion of observables for the $|\varphi|^{4}$ model is studied in [39].

\section{Reduction to a key theorem}

In this section, we reduce the proofs of Theorems 1.11 and 1.13, as well as Propositions 1.12 and 1.16, to the key result Theorem 2.2. We also prove Theorem 1.10 concerning the map $R_{+}$. Finally, we prove Theorem 1.19, by transferring the finite volume statements of Theorem 1.11 to infinite volume. The proof of Theorem 2.2 is substantial and is carried out in the remainder of the paper.

\subsection{Flow of coupling constants}

We now prove Theorem 1.10, which concerns the map $R_{+}$. The proof uses the fact, proved in [19, Proposition 1.18], that if $Y \subset X \in \mathcal{S}_{j}$ and $F \in \mathcal{N}\left(X^{\square}\right)$ then

$$
\left\|\operatorname{Loc}_{X, Y} F\right\|_{T_{0}} \leq C\|F\|_{T_{0}} .
$$

It also uses the fact that for a block $B \in \mathcal{B}$ and small $r_{Q}>0$, the reciprocal $I(V, B)^{-1}$ of $I(V, B)$ is an analytic function of $V \in B_{\mathcal{Q}^{(0)}}\left(r_{Q}\right)$ taking values in $\mathcal{N},\|\cdot\|_{T_{0}}$. This and related facts are further discussed in Section 3.3, for the study of $K_{+}$.

A basic tool we use is the Cauchy estimate for analytic functions on Banach spaces, to infer estimates on the derivatives of an analytic function from estimates on the function itself. Cauchy's formula can be found in [35, p. 134]. For complex Banach spaces $X, Y, f$ an analytic map from a domain in $X$ to $Y$, a positive integer $p$ and directions $\dot{x}=\left(\dot{x}_{1}, \ldots, \dot{x}_{p}\right)$ in $X^{p}$, it follows from Cauchy's formula that the Fréchet derivative of order $p$ of $f$ is given by

$$
D^{p} f(x) \dot{x}=\oint \ldots \oint f\left(x+\sum t_{j} \dot{x}_{j}\right) \frac{d t_{1}}{2 \pi i t_{1}^{2}} \ldots \frac{d t_{p}}{2 \pi i t_{p}^{2}},
$$

where the contours of integration are circles in the complex plane, whose radius $r$ is such that the polydisc

$$
\left\{x+\sum_{j} t_{j} \dot{x}_{j}:\left|t_{j}\right|<r, j=1, \ldots, p\right\}
$$

is contained in the domain of $f$. The Cauchy estimate follows from Cauchy's formula in the same way that it does for analytic functions of a single variable, and can be found explicitly in [22, Theorem 9.16].

The following proposition is used in our proofs of continuity statements in our main results, e.g., in Theorems 1.101 .11 , 
Proposition 2.1. Let $X$ and $Y$ be Banach spaces and let $U$ be an open subset of $X$. Let $E$ be a compact topological space. Let $f:(s, x) \mapsto f_{s}(x)$ be a uniformly bounded map from $E \times X$ to $Y$ such that $x \mapsto f_{s}(x)$ is analytic and $s \mapsto f_{s}(x)$ is continuous. Then for $p \in \mathbb{N}_{0}$, the map $(s, x, \dot{x}) \mapsto D^{p} f_{s}(x) \dot{x}$ from $E \times U \times X^{p}$ to $Y$ is jointly continuous.

Proof. Let $x \in U$ and $p \in \mathbb{N}_{0}$. By the uniform bound on $f$, and by (2.2), for directions $\dot{x}$ of unit norm, the multilinear map $D^{p} f_{s}(y)$ is bounded in norm uniformly both in $s$ and in $y$ in some neighbourhood $U_{x}$ of $x$. Also, by the Cauchy integral formula and dominated convergence, the map $s \mapsto D^{p} f_{s}(x) \dot{x}$ is continuous in $s$. Since $D^{p} f_{s}(y)$ is itself differentiable, and the Fréchet derivative is also bounded uniformly, the map $y \mapsto D^{p} f_{s}(y)$ is norm continuous on $U_{x}$ uniformly in $s$.

We demonstrate the case $p=1$, and omit the proof for $p=0$ as well as the inductive proof for $p>1$. Let $p=1, s, t \in E, x, y \in U$ and $\dot{x}, \dot{y} \in X$. We must show that $D f_{t}(y) \dot{y}$ converges to $D f_{s}(x) \dot{x}$ as $(t, y, \dot{y})$ tends to $(s, x, \dot{x})$, and we start with

$$
D f_{t}(y) \dot{y}-D f_{s}(x) \dot{x}=D f_{t}(y)(\dot{y}-\dot{x})+\left(D f_{t}(y) \dot{x}-D f_{t}(x) \dot{x}\right)+\left(D f_{t}(x) \dot{x}-D f_{s}(x) \dot{x}\right) .
$$

The first term tends to zero because $D f_{t}(y)$ is bounded in norm uniformly in $y$ near $x$. The second term tends to zero because $y \mapsto D f_{t}(y)$ is norm continuous at $y=x$ uniformly in $t$. The third term tends to zero because $t \mapsto D f_{t}(x) \dot{x}$ is continuous at $t=s$. This concludes the proof for $p=1$.

Proof of Theorem 1.10. By (1.75),

$$
R_{+}(V, K)=V_{\mathrm{pt}}^{(1)}(V-Q)-V_{\mathrm{pt}}^{(1)}(V)
$$

with

$$
Q(B)=\sum_{Y \in \mathcal{S}: Y \supset B} \operatorname{Loc}_{Y, B} I^{-Y} K(Y)
$$

The map $V_{\mathrm{pt}}$ is quadratic in $V$ by definition, and hence entire analytic in $V$. The map Loc $\operatorname{Loc}_{Y, B}$ is a bounded linear map (with respect to the $\|\cdot\|_{T_{0}}$ norm), by (2.1), and, as noted above, $I^{-Y}$ is analytic in $V \in B_{\mathcal{Q}^{(0)}}\left(r_{Q}\right)$. Therefore $Q$ is analytic in $V \in B_{\mathcal{Q}^{(0)}}\left(r_{Q}\right)$ and linear in $K$, and hence $R_{+}$is analytic in $V \in B_{\mathcal{Q}^{(0)}}\left(r_{Q}\right)$ and quadratic in $K$. It is also continuous in $m^{2} \in[0, \delta]$, since the coefficients of $V_{\mathrm{pt}}$ (given explicitly in [7, (3.30)-(3.35)]) are continuous in $m^{2} \in[0, \delta]$ by [7, Proposition 4.4].

Next we prove the estimates of (1.53), which we repeat here as

$$
\left\|D_{V}^{p} D_{K}^{q} R_{+}\right\|_{L^{p, q}} \leq M \begin{cases}r_{0} r_{Q}^{-p} & p \geq 0, q=0 \\ r_{Q}^{1-p-q} & p \geq 0, q=1,2 \\ 0 & p \geq 0, q \geq 3 .\end{cases}
$$

The $q \geq 3$ case of (2.7) holds since $R_{+}$is quadratic in $K$. For the remaining cases of (2.7), we use the Cauchy estimate for analytic functions. Recall from (1.34) that the $\mathcal{Q}$ norm is equivalent to the $T_{0}$ norm on a block. We choose $r_{Q}$ small enough that $Q$ is analytic in $V \in B_{\mathcal{Q}^{(0)}}\left(2 r_{Q}\right)$ and $\left\|I(V)^{-Y}\right\|_{T_{0}} \leq 2$ for $Y \in \mathcal{S}$. Then $\|Q(B)\|_{T_{0}(\ell)} \leq O\left(r_{0}\right)$ for $K \in B_{T_{0}}\left(2 r_{Q}\right)$. It follows from a small extension of [20, Proposition 1.5] that $\left\|V_{\mathrm{pt}}^{(1)}(V-Q, B)\right\|_{T_{0}(\ell)} \leq O\left(r_{Q}\right)$. Let $\dot{K}$ have unit $T_{0}$ norm, 
and let $Q_{t}=Q(K+t \dot{K})$ and $f(t)=V_{\mathrm{pt}}^{(1)}\left(V-Q_{t}(B)\right)$. Then $\|f(t)\|_{T_{0}} \leq O\left(r_{Q}\right)$, and $f(t)$ is analytic in $t \in \mathbb{C}$, as long as $K+t \dot{K} \in B_{T_{0}}\left(2 r_{Q}\right)$. We seek estimates for $K$ in the smaller ball of radius $r_{Q}$, so $f(t)$ is analytic in $t$ for $|t|<2 r_{Q}-r_{Q}=r_{Q}$. By the Cauchy estimate, for $K$ in the smaller ball we have

$$
\left\|D_{K} V_{\mathrm{pt}}^{(1)}(V-Q, B) \dot{K}\right\|_{T_{0,+}}=\left\|f^{\prime}(0)\right\|_{T_{0,+}} \leq \frac{O\left(r_{Q}\right)}{2 r_{Q}-r_{Q}}=O(1) .
$$

By taking the supremum over $\dot{K}$ we obtain the $(p, q)=(0,1)$ case of (2.7).

For the $(p, q)=(0,0)$ case of (2.7) , we define $g(s)=V_{\mathrm{pt}}^{(1)}(V-Q(s K), B)$ with $K \in B_{T_{0}}\left(r_{0}\right)$, so that $R_{+}(B)=\int_{0}^{1} g^{\prime}(s) d s$. Application of the previous Cauchy argument to bound $g^{\prime}(s)$ leads to $\left\|R_{+}(B)\right\|_{T_{0}} \leq O\left(r_{0}\right)$ on $B_{\mathcal{Q}^{(0)}}\left(2 r_{Q}\right) \times B_{T_{0}}\left(r_{0}\right)$ (with $\left.r_{0} \leq r_{Q}\right)$. For $(p, q)=(0,2)$, as in (2.8) where we lost a factor $r_{Q}$ for the $K$-derivative in the Cauchy estimate, in a second application of the Cauchy estimate we lose another $r_{Q}$ and obtain $\left\|D_{K}^{2} V_{\mathrm{pt}}^{(1)}(V-Q, B)\right\|_{T_{0}} \leq O\left(r_{Q}^{-1}\right)$. This completes the proof of the $p=0$ case of (2.7).

We bound the $V$ derivatives similarly, using the fact that a distance $r_{Q}$ separates the ball $B_{\mathcal{Q}^{(0)}}\left(r_{Q}\right)$ from the boundary of the larger ball $B_{\mathcal{Q}^{(0)}}\left(2 r_{Q}\right)$. For each $V$ derivative, the Cauchy estimate causes one power of $r_{Q}$ to be lost. This is the origin of the $r_{Q}^{-p}$ in (2.7), and this completes the proof of (2.7).

Finally, in view of the analyticity and continuity established in the first paragraph of the proof, the joint continuity of $D_{V}^{p} D_{K}^{q} R_{+}$follows from the uniform bound on $R_{+}$and Proposition 2.1 .

\subsection{Flow of non-perturbative coordinate}

In this section, we state Theorem 2.2 and show that it implies the statements concerning $K_{+}$in Theorem 1.13 and hence also Theorem 1.11. We include the statements of Propositions 1.12 and 1.16 as part of Theorem 2.2, so as to combine what must be proved about the finite volume $K_{+}$in one place. Theorem 2.2 is stated in terms of $\mathcal{F}$ norms, and subsequently we show that estimates in terms of $\mathcal{F}$ can be combined to produce estimates in terms of $\mathcal{W}$ as in Theorems 1.11 and 1.13. The structure of the proof of Theorem 2.2 is discussed in Section 3.1 below; the proof is carried out in the remainder of the paper.

The following theorem holds for either of the norm pairs $\mathcal{F}=\mathcal{F}_{j}(G), \mathcal{F}_{+}=\mathcal{F}_{j+1}\left(T_{0}\right)$, or $\mathcal{F}=\mathcal{F}_{j}(\tilde{G}), \mathcal{F}_{+}=\mathcal{F}_{j+1}\left(\tilde{G}^{\gamma}\right)$. These spaces depend on parameters $\tilde{g}, m^{2}$. The map $K_{+}$asserted to exist in the theorem is an explicit function of $(V, K)$ which is the same for each of the norm pairs (on the intersection of the domains). An important element of Theorem 2.2 is the fact that $\kappa<1$, in fact $\kappa$ can be made as small as desired by taking $L$ large. This contractive property of the map $K_{+}$is an essential feature in our applications in 6, 9, Recall that $\bar{\epsilon}$ is given in (1.43).

Theorem 2.2. Let $\mathbb{V}=\Lambda$ and $j<N(\Lambda)$.

(i) Fix any $a \in\left(0,2^{-d}\right), C_{\mathcal{D}}$ (as large as desired), and let $L$ be sufficiently large. There exist $r$ (small, independent of $L$ ), $\delta$ (small, dependent on $r, L), M_{0}>0$ (large, dependent on $L$ ), $\gamma^{*}>0$ (large, independent of $L)$, such that for all $\tilde{g} \in(0, \delta)$ and $m^{2} \in \mathbb{I}_{j+1}$, and with $\kappa=\gamma^{*} L^{-1}$, there exists a map

$$
K_{+}: \mathcal{D} \times B_{\mathcal{F}}(r \bar{\epsilon}) \rightarrow B_{\mathcal{F}_{+}}\left(\kappa r \bar{\epsilon}_{+}\right),
$$


such that the expectation preserves the circle produce in the sense that (1.49) holds. The map $K_{+}$ is analytic in $(V, K)$, and

$$
\left\|K_{+}(V, 0)\right\|_{\mathcal{F}_{+}} \leq M_{0} \bar{\epsilon}_{+}^{3}
$$

Moreover, there exist $a_{+}>a$ and $\mathfrak{h}_{++}>\mathfrak{h}_{+}$such that (2.9)-(2.10) hold with a replaced by $a_{+}$and $\mathfrak{h}_{+}$replaced by $\mathfrak{h}_{++}$in the $k=j+1$ definitions (1.41) and (1.35) -(1.36).

(ii) For $U \in \mathcal{P}_{+}(\Lambda)$, the value of $K_{+}(U)$ depends on $K$ only via $K(X)$ for $X \in \mathcal{P}\left(U^{\square}\right)$.

(iii) Let $U \in \mathcal{P}_{+}(\Lambda)$ be a coordinate patch and let $\iota: U^{\square} \rightarrow \Lambda^{\prime}$ with $\Lambda^{\prime}$ larger than $\Lambda$. Let $g_{\Lambda}$ be the map defined above Proposition 1.16 (given by (2.9)). Then $\iota g_{\Lambda}\left(\left.K_{\Lambda}\right|_{U^{\square}}\right)=g_{\Lambda^{\prime}}\left(\iota\left(\left.K_{\Lambda}\right|_{U^{\square}}\right)\right.$ ).

(iv) Let $\tilde{m}^{2} \in \mathbb{I}_{j+1}$. The map $K_{+}$extends to a map

$$
K_{+}: \mathcal{D} \times B_{\tilde{\mathcal{F}}}(r \bar{\epsilon}) \times \tilde{\mathbb{I}}_{+}\left(\tilde{m}^{2}\right) \rightarrow B_{\tilde{\mathcal{F}}_{+}}\left(\kappa r \bar{\epsilon}_{+}\right)
$$

which is analytic in $(V, K)$, continuous in $m^{2} \in \tilde{\mathbb{I}}_{j+1}\left(\tilde{m}^{2}\right)$, and obeys (2.10). Here $\tilde{\mathcal{F}}$ is defined in terms of $\tilde{\chi}=\chi\left(\tilde{m}^{2}\right)$, whereas $m^{2}$ is the mass in the original covariance $\left(-\Delta+m^{2}\right)^{-1}$.

(v) For $x=a$ or $x=b$, if $\pi_{x} V=0$ and $\pi_{x} K(X)=0$ for all $X \in \mathcal{P}$ then $\pi_{x} K_{+}(U)$ for all $U \in \mathcal{P}_{+}$.

Definition 2.3. For later convenience, we refer to the analyticity statement of part (i), and to the statements of parts (ii,iii,iv), simply as $(V, K)$-analyticity, the restriction property, the isometry property, and mass continuity, respectively. There is also a vanishing at weighted infinity property of $K_{+}$inherent in the definition of $\mathcal{F}_{+}$(see (1.37) ), and the field locality, symmetry and component factorisation properties of $K_{+}$inherent in the definition of $\mathcal{K}_{+}$in Definition 1.7. We use these terms when verifying these eight properties of $K_{+}$in later sections.

Our next goal is to conclude our main results for the finite volume $K_{+}$, from Theorem 2.2. The statement of Theorem 2.2 includes the statements of Propositions 1.12 and 1.16, and we show now that Theorems 1.11 and 1.13 follow from Theorem 2.2. This requires the conversion of $\mathcal{F}$ estimates to $\mathcal{W}$ estimates.

Let $\omega=\tilde{g}^{3 / 4}$, as in (1.44). We begin with the following lemma, which uses the $\mathcal{Y}_{+}$norm defined by

$$
\|K\|_{\mathcal{Y}_{+}}=\max \left\{\|K\|_{\mathcal{F}_{+}\left(T_{0}\right)}, \omega_{+}^{3}\|K\|_{\mathcal{F}_{+}(\tilde{G})}\right\}
$$

Lemma 2.4. There is a constant $c_{9}>0$ such that for any $K \in \mathcal{K}_{+}$,

$$
\|K\|_{\mathcal{W}_{+}} \leq c_{9}\|K\|_{\mathcal{Y}_{+}}
$$

Proof. It follows from [18, Proposition 3.17], for $X \in \mathcal{P}_{+}, K(X) \in \mathcal{N}(X)$, and for any positive integer $A<p_{\mathcal{N}}$, that there is a constant $c_{A}$ such that

$$
\|K(X)\|_{G_{+}, \ell_{+}} \leq c_{A} \max \left\{\|K(X)\|_{T_{0,+}\left(\ell_{+}\right)},\left(\frac{\ell_{+}}{h_{+}}\right)^{A+1}\|K(X)\|_{\tilde{G}_{+}, h_{+}}\right\} .
$$

We apply (2.14) with $A=9$; it is for this reason that we require $p_{\mathcal{N}} \geq 10$. To account for observables, the ratio $\ell_{+} / h_{+}$here is understood as the maximum of the two ratios $\ell_{j+1} / h_{j+1}$ and $\ell_{\sigma, j+1} / h_{\sigma, j+1}$. By (1.31)-(1.32), both ratios are bounded above by an $L$-dependent multiple of $\tilde{g}_{+}^{1 / 4}$. This gives

$$
\|K(X)\|_{G_{+}, \ell_{+}} \leq c_{9} \max \left\{\|K(X)\|_{T_{0,+}, \ell_{+}}, c(L) \tilde{g}_{+}^{10 / 4}\|K(X)\|_{\tilde{G}_{+}, h_{+}}\right\}
$$


As discussed below (1.46), we make the choice $\tilde{a}=4 a$, and this choice gives

$$
\begin{aligned}
& \bar{\epsilon}_{+}(\ell)^{f_{+}(a, X)}=\left(\chi_{+}^{1 / 2} \tilde{g}_{+}\right)^{a\left(|X|-2^{d}\right)_{+}} \\
& \quad \geq\left(\chi_{+}^{1 / 2} \tilde{g}_{+}^{1 / 4}\right)^{4 a\left(|X|-2^{d}\right)_{+}}=\bar{\epsilon}_{+}(h)^{f_{+}(\tilde{a}, X)} .
\end{aligned}
$$

With (1.41), this implies that

$$
\|K\|_{\mathcal{F}_{+}(G)} \leq c_{9} \max \left(\|K\|_{\mathcal{F}_{+}\left(T_{0}\right)}, c(L) \tilde{g}_{+}^{10 / 4}\|K\|_{\mathcal{F}_{+}(\tilde{G})}\right)
$$

and since $c(L) \tilde{g}_{+}^{10 / 4} \leq \tilde{g}_{+}^{9 / 4}=\omega_{+}^{3}$ for $\tilde{g}_{+}$sufficiently small depending on $L$, this implies that

$$
\|K\|_{\mathcal{W}_{+}} \leq c_{9}\|K\|_{\mathcal{Y}_{+}}
$$

This completes the proof.

We now show that Theorems 1.11 and 1.13 follow from Theorem 2.2. We begin with Theorem 1.11, and afterwards consider the mass continuity statement of Theorem 1.13.

Proof of Theorem 1.11. Fix $R>0$ (large). The proof uses $\mathcal{W}$ balls of radii $a \ll A$, defined in terms of small $r$ and large $R$ by

$$
a=R \chi^{3 / 2} \tilde{g}^{3}, \quad A=r \chi^{1 / 2} \tilde{g}^{10 / 4} .
$$

We use analyticity in the ball of larger radius to prove estimates in the ball of smaller radius, using Cauchy estimates. The radius $a$ appears in the definition (1.56) of $\mathbb{D}_{j}(\Lambda)$ and $A$ is chosen so that $\omega^{-3} A=r \bar{\epsilon}(h)$ where $\omega$ is defined by (1.44).

For $K$ in the larger ball $B_{\mathcal{W}}(A)$ of radius $A$, the definition (1.45) of the $\mathcal{W}$ norm translates into the $\mathcal{F}$ estimates

$$
\|K\|_{\mathcal{F}(G)} \leq A \leq r \bar{\epsilon}(\ell), \quad\|K\|_{\mathcal{F}(\tilde{G})} \leq \omega^{-3} A=r \bar{\epsilon}(h)
$$

and the inclusions

$$
B_{\mathcal{W}}(A) \subset B_{\mathcal{F}(G)}(A), \quad B_{\mathcal{W}}(A) \subset B_{\mathcal{F}(\tilde{G})}\left(\omega^{-3} A\right) .
$$

Recall that Theorem 2.2 asserts that $(K, V) \mapsto K_{+}$is analytic (continuously differentiable) as a map from $\mathcal{D} \times \mathcal{F}$ to $\mathcal{F}_{+}$for two choices of the pair $\left(\mathcal{F}, \mathcal{F}_{+}\right)$, namely $\left(\mathcal{F}(G), \mathcal{F}_{+}\left(T_{0}\right)\right)$ and $\left(\mathcal{F}(\tilde{G}), \mathcal{F}_{+}(\tilde{G})\right)$. Since the inclusions (2.21) are bounded linear maps they are analytic. Therefore, the composition of these inclusions with $(K, V) \mapsto K_{+}$is analytic. It follows that $(K, V) \mapsto K_{+}$ is an analytic map from $\mathcal{D} \times B_{\mathcal{W}}(A)$ into the intersection of the two choices of $\mathcal{F}_{+}$, which is the space $\mathcal{Y}$ defined by (2.12). According to Lemma 2.4, $\mathcal{Y}$ is continuously embedded into $\mathcal{W}_{+}$, so with a further composition with this embedding we find that $(K, V) \mapsto K_{+}$is an analytic map from $\mathcal{D} \times B_{\mathcal{W}}(A)$ to $\mathcal{W}_{+}$. Since $\mathcal{D} \times B_{\mathcal{W}}(A)$ contains $\mathbb{D}$ we have proved that $(K, V) \mapsto K_{+}$is analytic on $\mathbb{D}$ as claimed in Theorem 1.11.

Our first task is to prove case $(p, q)=(0,1)$ of the estimates claimed in Theorem 1.11 for $(V, K) \in \mathbb{D}$, namely

$$
\left\|D_{V}^{p} D_{K}^{q} K_{+}(V, K)\right\|_{L^{p, q}} \leq \begin{cases}\kappa^{\prime \prime} & p=0, q=1 \\ M^{\prime \prime} \chi_{+}^{3 / 2} \tilde{g}_{+}^{3-p} & p \geq 0 \\ M^{\prime \prime} \tilde{g}_{+}^{-p}\left(\chi_{+}^{1 / 2} \tilde{g}_{+}^{10 / 4}\right)^{1-q} & p \geq 0, q \geq 1\end{cases}
$$


We will prove that case $(p, q)=(0,1)$ holds on the larger domain $\mathcal{D} \times B_{\mathcal{W}}(A / 2)$ and this stronger statement is used in the proof of the other cases.

Let $(V, K) \in \mathcal{D} \times B_{\mathcal{W}}(A / 2)$. Let $\dot{K} \in \mathcal{F}$ and set $T=D_{K} K_{+}(V, K)$. We first prove that

$$
\|T \dot{K}\|_{\mathcal{F}_{+}} \leq \kappa^{\prime}\|\dot{K}\|_{\mathcal{F}}
$$

where $\kappa^{\prime}=O\left(L^{-1}\right)$. The argument is the same for both norm pairs. We start with the pair $\mathcal{F}=\mathcal{F}(\tilde{G})$ and $\mathcal{F}_{+}=\mathcal{F}_{+}(\tilde{G})$. Let $f(t)=K_{+}(V, K+t \dot{K})$. By Theorem $2.2 f$ is analytic at $t \in \mathbb{C}$ such that $K+t \dot{K}$ is in the ball $B_{\mathcal{F}}(r \bar{\epsilon}(h))$, and $f(t)$ has values in the ball $B_{\mathcal{F}_{+}}\left(\kappa r \bar{\epsilon}_{+}(h)\right)$. Since $K$ is in the smaller ball $B_{\mathcal{W}}(A / 2) \subset B_{\mathcal{F}}(r \bar{\epsilon}(h) / 2), f(t)$ is analytic in $t$ for $|t|<r \bar{\epsilon}(h) / 2$. By the Cauchy estimate,

$$
\|T \dot{K}\|_{\mathcal{F}_{+}}=\left\|f^{\prime}(0)\right\|_{\mathcal{F}_{+}} \leq \frac{\sup _{t}\|f(t)\|_{\mathcal{F}_{+}}}{r \bar{\epsilon}(h)-r \bar{\epsilon}(h) / 2} \leq \frac{\kappa r \bar{\epsilon}_{+}(h)}{r \bar{\epsilon}(h) / 2}=O(\kappa),
$$

where the last equality follows from (1.30). By Theorem $2.2, \kappa=O\left(L^{-1}\right)$. Since $T$ is a linear operator, the above bound on unit norm $\dot{K}$ implies (2.23) for this norm pair.

Now we consider the same argument for the other norm pair, $\mathcal{F}=\mathcal{F}(G)$ and $\mathcal{F}_{+}=\mathcal{F}_{+}\left(T_{0}\right)$. This time $f(t)$ is analytic in $t$ for $|t|<r \bar{\epsilon}(\ell)-A / 2$ and the right-hand side of the Cauchy estimate is $\kappa r \bar{\epsilon}_{+}(\ell) /(r \bar{\epsilon}(\ell)-A / 2)$. Since $A=O\left(\chi^{1 / 2} \tilde{g}^{10 / 4}\right)$, it is negligible relative to $r \bar{\epsilon}(\ell)$, which is given by (1.43), and we have proved (2.23) for both norm pairs.

By (2.23)

$$
\begin{gathered}
\|T \dot{K}\|_{\mathcal{F}_{+}\left(T_{0}\right)} \leq \kappa^{\prime}\|\dot{K}\|_{\mathcal{F}(G)} \leq \kappa^{\prime}\|\dot{K}\|_{\mathcal{W}}, \\
\omega_{+}^{3}\|T \dot{K}\|_{\mathcal{F}_{+}(\tilde{G})} \leq \omega_{+}^{3} \kappa^{\prime}\|\dot{K}\|_{\mathcal{F}(\tilde{G})} \leq 2 \kappa^{\prime}\|\dot{K}\|_{\mathcal{W}}
\end{gathered}
$$

where we used $\omega_{+} \leq 2 \omega$. We combine this with Lemma 2.4 and the definition (2.12) of $\mathcal{Y}$, to obtain

$$
\|T \dot{K}\|_{\mathcal{W}_{+}} \leq c_{9}\|T \dot{K}\|_{\mathcal{Y}_{+}} \leq \kappa^{\prime \prime}\|\dot{K}\|_{\mathcal{W}}
$$

where $\kappa^{\prime \prime}=2 c_{9} \kappa^{\prime}$. We have proved (2.27) for $(V, K) \in \mathcal{D} \times B_{\mathcal{W}}(A / 2)$, which is a larger domain than $\mathbb{D}$. Since $T=D_{K} K_{+}(V, K)$, this proves case $(p, q)=(0,1)$ of (2.22).

Next we prove case $(p, q)=(0,0)$ of (2.22). By integrating the $t$ derivative of $K_{+}(V, t K)$ with respect to $t$ and estimating the integrand with (2.27), we have, for $K$ in $B_{\mathcal{W}}(A / 2)$,

$$
\left\|K_{+}(V, K)-K_{+}(V, 0)\right\|_{\mathcal{W}_{+}} \leq \kappa^{\prime \prime}\|K\|_{\mathcal{W}}
$$

Furthermore, it follows from (2.10) and Lemma 2.4 that

$$
\begin{aligned}
\left\|K_{+}(V, 0)\right\|_{\mathcal{W}_{+}} & \leq c_{9}\left\|K_{+}(V, 0)\right\|_{\mathcal{Y}_{+}} \leq c_{9} \max \left\{M_{0} \chi_{+}^{3 / 2} \tilde{g}_{+}^{3}, \tilde{g}_{+}^{9 / 4} M_{0} \chi_{+}^{3 / 2} \tilde{g}_{+}^{3 / 4}\right\} \\
& =c_{9} M_{0} \chi_{+}^{3 / 2} \tilde{g}_{+}^{3} .
\end{aligned}
$$

Now let $K$ be in the small ball $B_{a}(\mathcal{W})$ required for case $(p, q)=(0,0)$. By combining (2.28) $-(2.29)$, we obtain

$$
\left\|K_{+}(V, K)\right\|_{\mathcal{W}_{+}} \leq c_{9} M_{0} \chi_{+}^{3 / 2} \tilde{g}_{+}^{3}+\kappa^{\prime \prime}\|K\|_{\mathcal{W}} \leq c_{9}\left(M_{0}+\kappa R^{\prime}\right) \chi_{+}^{3 / 2} \tilde{g}_{+}^{3}=M_{1} \chi_{+}^{3 / 2} \tilde{g}_{+}^{3},
$$


where $R^{\prime}$ is a multiple of $R$, we take $L$ large enough that $\kappa R^{\prime} \leq 1$, and $M_{1}=c_{9}\left(M_{0}+1\right)$. This proves case $(p, q)=(0,0)$ of (2.22). For later use, note that (2.28) $-(2.29)$ imply that $K_{+}$maps $\mathcal{D} \times B_{\mathcal{W}}(A / 2)$ into $B_{\mathcal{W}_{+}}(A)$.

To obtain (2.22) for the case $q=0, p>0$, we fix $K \in B_{\mathcal{W}}(a)$, for which we have just established that $K_{+} \in B_{\mathcal{W}_{+}}\left(M_{1} \chi^{3 / 2} \tilde{g}^{3}\right)$. This bound is understood to hold for $V \in \mathcal{D}\left(2 C_{\mathcal{D}}\right)$; this is the domain $\mathcal{D}$ with $C_{\mathcal{D}}$ doubled, and since $C_{\mathcal{D}}$ is arbitrary in Theorem 2.2. we can use its conclusions with the doubled value. Let $\left\|\dot{V}_{i}\right\|_{\mathcal{Q}}=1$, and let $f(s)=K_{+}\left(V+\sum_{i} s_{i} \dot{V}_{i}, K\right)$. We choose $\epsilon>0$ so that $V+\sum_{i} s_{i} \dot{V}_{i} \in \mathcal{D}\left(2 C_{\mathcal{D}}\right)$ for $\left|s_{i}\right| \leq \epsilon \tilde{g}$. Then we apply the Cauchy estimate to $f$ as an analytic function of $s_{1}, \ldots, s_{p}$ in the domain $\left|s_{i}\right|<\epsilon \tilde{g}$. The denominator in the analogue of (2.24) is the distance from $V \in \mathcal{D}$ to the boundary of the domain $\mathcal{D}\left(2 C_{\mathcal{D}}\right)$, which is at least $\epsilon \tilde{g}$. For $p$ derivatives there is one such denominator for each derivative. This gives a factor proportional to $\tilde{g}_{j}^{-p}$ so the Cauchy estimate bounds the derivative by $O\left(\chi^{3 / 2} \tilde{g}^{3} \tilde{g}_{j}^{-p}\right)$ as stated in the second estimate of (2.22).

To obtain (2.22) for the case $p \geq 1, q \geq 1$, we use the Cauchy estimate on $f(s, t)=K_{+}(V+$ $\left.\sum s_{i} \dot{V}_{i}, K+\sum t_{i} \dot{K}_{i}\right)$ as an analytic function of $s_{1}, \ldots, s_{p}$ and $t_{1}, \ldots, t_{q}$ in the domain $\left|s_{i}\right|<\epsilon \tilde{g}$ and $\left|t_{i}\right|<\frac{1}{2} A$. The denominator in the analogue of (2.24) is the distance from $(V, K)$ to the boundary of the domain, and for $p+q$ derivatives there is one such denominator for each derivative, which gives a factor proportional to $\tilde{g}^{-p} A^{-q}$. As we have proved above the image of this domain lies in $B_{\mathcal{W}_{+}}(A)$, so the Cauchy estimate bounds the derivative by $O\left(\tilde{g}^{-p} A^{1-q}\right)$ as desired.

Proof of Theorem 1.13. By Theorem 2.2(iv), $K_{+}$is a continuous function of $m^{2} \in \tilde{\mathbb{I}}\left(\tilde{m}^{2}\right)$ as a map into $\tilde{\mathcal{F}}_{+}$. This is the case for each of the norm pairs, so $\tilde{\mathcal{F}}_{+}$can be either $\tilde{\mathcal{F}}_{+}\left(T_{0}\right)$ or $\tilde{\mathcal{F}}_{+}(\tilde{G})$. Therefore $K_{+}$is continuous as a map into the space $\tilde{\mathcal{Y}}_{+}$defined as in (2.12), and so by Lemma 2.4 it is continuous also as a map into $\tilde{\mathcal{W}}_{+}$. The joint continuity of $D_{V}^{p} D_{K}^{q} K_{+}$follows from the uniform bound on $K_{+}$and Proposition 2.1 .

\subsection{Proof of main result for infinite volume}

We now deduce our main result Theorem 1.19 for the infinite volume map, from the finite volume result Theorem 2.2. The proof for $R_{+, \mathbb{Z}^{d}}$ is similar to but simpler than the proof for $K_{+, \mathbb{Z}^{d}}$, and we only present the details for $K_{+, \mathbb{Z}^{d}}$. In Section 2.2, it is shown that the statements of Theorem 1.11 for $K_{+}$in finite volume are a consequence of Theorem 2.2(i,iv). The sufficiency of Theorem 2.2(i,iv) was established via Cauchy estimates based on analyticity, together with an argument to conclude estimates in $\mathcal{W}$ norm from those in $\mathcal{F}$ norm. Joint continuity of the Fréchet derivatives was a consequence of Proposition 2.1. These items apply in the same way to an infinite volume version of Theorem 2.2(i,iv), so it suffices to prove such an infinite volume version. This is the content of Theorem 2.5 below.

The infinite volume version of Theorem 2.2(iii) is omitted because it is meaningless in the infinite volume context. We do not need the infinite volume version of Theorem 2.2(ii), but we note that it does hold by the definition of $K_{+, \mathbb{Z}^{d}}$. Namely, for $U \in \mathcal{P}_{+}\left(\mathbb{Z}^{d}\right)$, the value of $K_{+, \mathbb{Z}^{d}}(U)$ depends on $K$ only via $K(X)$ for $X \in \mathcal{P}\left(U^{\square}\right)$. In addition, the field locality and symmetry properties for $K_{+, \mathbb{Z}^{d}}$, required for membership in $\mathcal{F}_{+}\left(\mathbb{Z}^{d}\right)$, follow from the corresponding finite volume properties by definition (1.88) of $K_{+, \mathbb{Z}^{d}}$. 
Theorem 2.5. Let $j=0,1, \ldots$ be any scale. In (1.36) set $\gamma=1$.

(i) Fix any $a \in\left(0,2^{-d}\right), C_{\mathcal{D}}$ (as large as desired), and let $L$ be sufficiently large. There exist $r$ (small, independent of $L$ ), $\delta$ (small, dependent on $r, L), M_{0}>0$ (large, dependent on $L$ ), $\gamma^{*}>0$ (large, independent of $L$ ), such that for all $\tilde{g}_{j} \in(0, \delta)$ and $m^{2} \in \mathbb{I}_{j+1}$, and with $\kappa=\gamma^{*} L^{-1}$,

$$
K_{+, \mathbb{Z}^{d}}: \mathcal{D} \times B_{\mathcal{F}\left(\mathbb{Z}^{d}\right)}(r \bar{\epsilon}) \rightarrow B_{\mathcal{F}_{+}\left(\mathbb{Z}^{d}\right)}\left(\kappa r \bar{\epsilon}_{+}\right) .
$$

The map $K_{+, \mathbb{Z}^{d}}$ is analytic in $(V, K)$, and

$$
\left\|K_{+, \mathbb{Z}^{d}}(V, 0)\right\|_{\mathcal{F}_{+}} \leq M_{0} \bar{\epsilon}_{+}^{3} .
$$

(iv) Let $\tilde{m}^{2} \in \mathbb{I}_{j+1}$. The map $K_{+, \mathbb{Z}^{d}}$ extends to a map

$$
K_{+, \mathbb{Z}^{d}}: \mathcal{D} \times B_{\tilde{\mathcal{F}}}(r \bar{\epsilon}) \times \tilde{\mathbb{I}}_{+}\left(\tilde{m}^{2}\right) \rightarrow B_{\tilde{\mathcal{F}}_{+}}\left(\kappa r \bar{\epsilon}_{+}\right),
$$

which is analytic in $(V, K)$, continuous in $m^{2} \in \tilde{\mathbb{I}}_{j+1}\left(\tilde{m}^{2}\right)$, and obeys (2.32). Here $\tilde{\mathcal{F}}$ is defined in terms of $\tilde{\chi}=\chi\left(\tilde{m}^{2}\right)$, whereas $m^{2}$ is the mass in the original covariance $\left(-\Delta+m^{2}\right)^{-1}$.

The proof relies on the facts about coordinate maps $\iota$ and extensions by symmetry given in Lemmas E.4 E.6.

Proof. (i) Let $\mathfrak{r}=r \bar{\epsilon}, \mathfrak{r}_{+}=\kappa r \bar{\epsilon}_{+}, W(X)=\bar{\epsilon}^{f(a, X)}, W_{+}(X)=\bar{\epsilon}_{+}^{f_{+}(a, X)}$. Let $(V, K) \in \mathcal{D} \times B_{\mathcal{F}\left(\mathbb{Z}^{d}\right)}(\mathfrak{r})$, so that (recall the definitions (1.41) and (1.35)-(1.36) )

$$
\|K(X)\|_{\mathcal{G}} \leq \mathfrak{r} W(X) \quad\left(X \in \mathcal{C}\left(\mathbb{Z}^{d}\right)\right),
$$

with $\mathcal{G}$ equal to $G_{j}$ or $\tilde{G}_{j}$. We will prove that $K_{+, \mathbb{Z}^{d}} \in B_{\mathcal{F}_{+}\left(\mathbb{Z}^{d}\right)}\left(\kappa r \bar{\epsilon}_{+}\right)$. Since we set $\gamma=1$, it is equivalent to prove that

$$
\left\|K_{+, \mathbb{Z}^{d}}(U)\right\|_{T_{0,+}} \leq \mathfrak{r}_{+} W_{+}(U), \quad\left\|K_{+, \mathbb{Z}^{d}}(U)\right\|_{\tilde{G}_{+}} \leq \mathfrak{r}_{+} W_{+}(U)
$$

hold for all connected polymers $U$ in $\mathbb{Z}^{d}$. We must also show that $K_{+, \mathbb{Z}^{d}}$ vanishes at weighted infinity (see (1.37)), since this is part of the definition of $\mathcal{F}_{+}$. In our present context, we must show that

$$
\lim _{\|\phi\|_{\Phi(U)} \rightarrow \infty}\left\|K_{+, \mathbb{Z}^{d}}(U)\right\|_{T_{\phi,+}} \tilde{G}_{+}^{-1}(U, \phi)=0 .
$$

Let $U$ be a connected polymer in $\mathbb{Z}^{d}$. We will construct $K_{+, \mathbb{Z}^{d}}(U)$ as the image of $K$ under a composition $\iota_{\Lambda}^{-1} \circ C_{U} \circ B \circ A$ of four maps.

Let $U^{\prime}=U^{\square}$, and let $\iota_{\Lambda}: U^{\prime} \rightarrow \Lambda$ be a coordinate map to a torus. By (2.34) and Lemma E.4,

$$
\left\|\iota_{\Lambda} K\left(\iota_{\Lambda}^{-1} X\right)\right\|_{\mathcal{G}} \leq \mathfrak{r} W(X) \quad\left(X \in \mathcal{C}\left(\iota_{\Lambda} U^{\prime}\right)\right) .
$$

Let $\mathcal{X}=\mathcal{C}\left(\iota_{\Lambda} U^{\prime}\right)$. By Lemma E.6, with Euclidean symmetry hypothesis verified as above (1.87), the map $X \mapsto \iota_{\Lambda} K\left(\iota_{\Lambda}^{-1} X\right)$ extends from $\mathcal{X}$ to an element $\hat{K} \in B_{\mathcal{F}(\Lambda)}(\mathfrak{r})$. Lemma E.6 implies that the map $A_{U}: K \mapsto \hat{K}$ preserves the vanishing at weighted infinity property and is a linear contraction from $\mathcal{F}\left(\mathbb{Z}^{d}\right)$ to $\mathcal{F}(\Lambda)$. In particular, the evaluation map is analytic. We next apply Theorem 2.5, with $a$ and $\mathfrak{h}_{+}$replaced by the values $a_{+}$and $\mathfrak{h}_{++}$provided by Theorem 2.5, To 
remind us that we have these stronger values, we write $\mathcal{F}_{++}$in place of $\mathcal{F}_{+}$and $\mathcal{N}_{++}$in place of $\mathcal{N}_{+}$. By Theorem 2.2, the map $B:(V, \hat{K}) \mapsto K_{+, \Lambda}(V, \hat{K})$ is analytic as a map from the $\mathfrak{r}$ ball in $\mathcal{F}(\Lambda)$ to the $\mathfrak{r}_{+}$ball in $\mathcal{F}_{++}(\Lambda)$. Now consider evaluation on the polymer $\iota_{\Lambda} U$ as a map $C_{U}: K_{+, \Lambda} \mapsto K_{+, \Lambda}\left(\iota_{\Lambda} U\right)$. By definition of the $\mathcal{F}_{++}(\Lambda)$ norm, this is a bounded linear map into the space $\mathcal{N}_{++}\left(\iota_{\Lambda} U^{\prime}\right)$, and we have the ++ analogue of (2.36). In particular it is analytic. Furthermore, by (1.88), the composition $C_{U} \circ B \circ A_{U}$ is the map $(V, K) \mapsto \iota_{\Lambda} K_{+, \mathbb{Z}^{d}}(U)$ because $K_{+, \Lambda}\left(\iota_{\Lambda} U\right)$ does not depend on the choice $\hat{K}$ of extension of $\iota_{\Lambda} K\left(\iota_{\Lambda}^{-1} X\right)$ off $\mathcal{X}$. In summary, we have proved that $(V, K) \mapsto \iota_{\Lambda} K_{+, \mathbb{Z}^{d}}(U)$ is analytic as a map from the $\mathfrak{r}$ ball in $\mathcal{F}\left(\mathbb{Z}^{d}\right)$ to $\mathcal{N}_{++}\left(\iota_{\Lambda} U^{\prime}\right)$, and putting the estimates together we have

$$
\left\|\iota_{\Lambda} K_{+, \mathbb{Z}^{d}}(U)\right\|_{\mathcal{G}_{++}} \leq \mathfrak{r}_{+} W_{++}(U) .
$$

We now pass to an estimate on $K_{+, \mathbb{Z}^{d}}(U)$, exploiting the fact that (2.38) holds for all $\Lambda$. According to Lemma E.5 with $\mathfrak{h}$ replaced by $\mathfrak{h}_{++}$, when $\Lambda$ is sufficiently large, the inverse $\iota_{\Lambda}^{-1}$ is a bounded linear operator from $\mathcal{N}_{++}\left(U^{\square}\right)$ to $\mathcal{N}_{+}\left(U^{\square}\right)$. This is the step where we use the parameter $\gamma<1$ for the regulator $\tilde{G}^{\gamma}$, and where we use $\mathfrak{h}_{++}>\mathfrak{h}_{+}$. We obtain

$$
\begin{aligned}
& \left\|K_{+, \mathbb{Z}^{d}}(U)\right\|_{T_{0,+}} \leq \mathfrak{r}_{+} W_{++}(U), \\
& \left\|K_{+, \mathbb{Z}^{d}}(U)\right\|_{\tilde{G}_{+}} \leq \mathfrak{r}_{+} W_{++}(U) .
\end{aligned}
$$

Therefore (2.35) holds and, since the bounded linear operator $\iota_{\Lambda}^{-1}$ is an analytic map from $\mathcal{N}_{++}\left(U^{\square}\right)$ to $\mathcal{N}_{+}\left(U^{\square}\right)$, we can compose with the previous maps and conclude that $(V, K) \mapsto \iota_{\Lambda} K_{+, \mathbb{Z}^{d}}(U)$ is analytic as a map from the $\mathfrak{r}$ ball in $\mathcal{F}\left(\mathbb{Z}^{d}\right)$ to $\mathcal{N}_{+}\left(U^{\prime}\right)$. Furthermore, (2.36) holds. Therefore $K_{+, \mathbb{Z}^{d}} \in B_{\mathcal{F}_{+}\left(\mathbb{Z}^{d}\right)}\left(\mathfrak{r}_{+}\right)$, as desired.

If we set $K=0$ so that $\hat{K}=0$ and use (2.10), namely $\left\|K_{+}(V, 0)\right\|_{\mathcal{F}_{+}} \leq M_{0} \bar{\epsilon}^{3}$, then an argument analogous to the one above gives (2.32).

We now strengthen the above analyticity, which is pointwise in $U$, to the desired analyticity statement. It is here that we take advantage of the fact that $a_{+}>a$. For a positive integer $M$, let $\mathcal{U}_{0}=\left\{U \in \mathcal{P}_{+}:|U|_{j+1} \leq M, U \ni 0\right\}$. Let $\mathcal{U}_{0}^{\prime}=\left\{U^{\prime} \in \mathcal{P}_{+}: U \in \mathcal{U}_{0}\right\}$. Let $g_{M, 0}: \mathcal{D} \times B_{\mathcal{F}\left(\mathbb{Z}^{d}\right)}(\mathfrak{r}) \rightarrow$ $\mathcal{N}_{+}^{\mathcal{U}_{0}^{\prime}}$ be the map that takes $(V, K)$ into $\left(\pi_{\varnothing} K_{+, \mathbb{Z}^{d}}(U), U \in \mathcal{U}_{0}\right)$. The latter is a Banach space with the norm

$$
\|F\|=\sup _{U \in \mathcal{U}_{0}}\|F(U)\|_{\mathcal{N}_{+}\left(U^{\prime}\right)} W_{++}^{-1}(U) .
$$

Since $\mathcal{U}_{0}$ is finite, it follows from Lemma 3.4 and the analyticity pointwise in $U$ that $g_{M, 0}$ is analytic. Let $g_{M}(V, K)=\pi_{\varnothing} K_{+, \mathbb{Z}^{d}} \mathbb{1}_{M}$, where $\mathbb{1}_{M}(U)=1$ if $|U|_{j+1} \leq M$ and otherwise is zero. For every polymer $U$ such that $|U|_{j+1} \leq M$, there exists a translation $E_{U}$ of $\mathbb{Z}^{d}$ such that $E_{U} U$ contains the origin. By the Euclidean invariance of $\pi_{\varnothing} K_{+, \mathbb{Z}^{d}}$,

$$
\pi_{\varnothing} K_{+, \mathbb{Z}^{d}}(U)=E_{U}^{-1} \pi_{\varnothing} K_{+, \mathbb{Z}^{d}}\left(E_{U} U\right) .
$$

These relations imply that $g_{M}$ equals the composition of $g_{M, 0}$ with a bounded linear extension map into the space $\mathcal{K}_{+}\left(\mathbb{Z}^{d}\right)$ with the norm

$$
\|F\|_{\mathcal{F}_{+}\left(W_{++}\right)}=\sup _{U \in \mathcal{C}_{+}\left(\mathbb{Z}^{d}\right)}\|F(U)\|_{\mathcal{N}_{+}\left(U^{\prime}\right)} W_{++}^{-1}(U)
$$

Therefore, for each $M$ the map $g_{M}$ is analytic as a map with values in the space $\mathcal{K}_{+}\left(\mathbb{Z}^{d}\right)$ with this norm. When $a_{+}$is replaced by $a$, the weight $W_{++}$becomes $W_{+}$, and this norm becomes the 
$\mathcal{F}_{+}\left(\mathbb{Z}^{d}\right)$ norm. Furthermore, since $a_{+}>a$, the space $\mathcal{F}_{+}\left(\mathbb{Z}^{d}\right)$ is a larger space, and uniformly in $V, K$ we have

$$
\lim _{M \rightarrow \infty}\left\|\pi_{\varnothing} K_{+, \mathbb{Z}^{d}}\left(1-\mathbb{1}_{M}\right)\right\|_{\mathcal{F}_{+}\left(\mathbb{Z}^{d}\right)}=0 .
$$

Therefore the sequence $g_{M}$ of analytic functions converges to $\pi_{\varnothing} K_{+, \mathbb{Z}^{d}}$ in $\mathcal{F}_{+}\left(\mathbb{Z}^{d}\right)$ uniformly in $K$ as $M \rightarrow \infty$. According to [35, Theorem 2, p. 137], as a uniform limit, $\pi_{\varnothing} K_{+, \mathbb{Z}^{d}}$ is analytic as a map into $\mathcal{F}_{+}\left(\mathbb{Z}^{d}\right)$. The observable component $\pi_{*} K_{+, \mathbb{Z}^{d}}$ is also analytic as a map into $\mathcal{F}_{+}\left(\mathbb{Z}^{d}\right)$ using the same argument with $\mathcal{U}_{0}$ replaced by $\mathcal{U}_{*}=\left\{U \in \mathcal{P}_{+}:|U|_{j+1} \leq M, U \cap\{a, b\} \neq \varnothing\right\}$ and omitting (2.42) and the line below about composition with an extension. Having proved that the $\varnothing$ and $*$ components of $K_{+, \mathbb{Z}^{d}}$ are analytic it follows from Lemma 3.4 that $K_{+, \mathbb{Z}^{d}}$ is analytic as a map into $\mathcal{F}_{+}\left(\mathbb{Z}^{d}\right)$. This concludes the proof of part (i).

(iv) Let $\tilde{m}^{2} \in \mathbb{I}_{j+1}$. We return to the map $B$ defined in part (i). By Theorem 2.2(iv), $B$ extends to a map that depends on $m^{2}$, and by fixing $(V, \hat{K})$ this extension becomes a continuous map

$m^{2} \mapsto K_{+, \Lambda}$ into $\tilde{\mathcal{F}}_{+}(\Lambda)$. The other maps $A_{U}, C_{U}, \iota_{\Lambda}^{-1}$ do not depend on $m^{2}$. It follows that the composition $m^{2} \mapsto K_{+, \mathbb{Z}^{d}}(U)$ is continuous as a map into $\mathcal{N}\left(U^{\prime}\right)$ with norms $\|\cdot\|_{T_{0,+}}$ and $\|\cdot\|_{\tilde{G}_{+}}$. Finally, if we replace $\mathcal{F}_{+}\left(\mathbb{Z}^{d}\right)$ by the $m^{2}$ independent space $\tilde{\mathcal{F}}_{+}\left(\mathbb{Z}^{d}\right)$, then the convergence in (2.44) is also uniform in $m^{2}$ and since the uniform limit of continuous functions is continuous, we also achieve mass continuity as claimed. This completes the proof.

\section{Preliminaries to proof of Theorem 2.2}

In Section 3.1, we describe the basic structure of the proof of Theorem [2.2, and in Section 3.2 we specify several parameters that occur in the proof. In Section 3.3, we recall several useful results from [20].

We assume throughout the paper that $\tilde{g}$ is sufficiently small to carry out each steps that is encountered. According to the definition of $\bar{\epsilon}$ in (1.43), taking $\tilde{g}$ small is equivalent to taking $\bar{\epsilon}$ small, and we often phrase smallness conditions in terms of $\bar{\epsilon}$ instead of $\tilde{g}$. The assumption that $\tilde{g}$ is small is used so frequently that we often apply it without explicit mention.

Throughout the paper, we use the following notation for non-negative real sequences $A=A_{j}$, $B=B_{j}$ :

$$
\begin{aligned}
A \prec B & \text { if } A_{j} \leq c B_{j} \text { for all } j, \text { with } c \text { independent of } L, \\
A \prec_{L} B & \text { if } A_{j} \leq c B_{j} \text { for all } j, \text { with } c=c(L), \\
A \asymp B & \text { if } A \prec B \text { and } B \prec A .
\end{aligned}
$$

\subsection{Structure of proof of Theorem 2.2}

We fix $V$ and regard the map $(V, K) \mapsto K_{+}$asserted to exist in Theorem 2.2 as a map $K \mapsto K_{+}$. We construct $K_{+}$as a composition of six maps:

$$
(\operatorname{Map} i): K^{(i-1)} \mapsto K^{(i)} i=1, \ldots, 6, \quad \text { with } K^{(0)}=K, \quad K^{(6)}=K_{+} .
$$

The six maps are described in detail in Sections 46, and are described briefly here. 
Maps 1 and 2 are defined in such a way that

$$
(I \circ K)(\Lambda)=\left(I \circ K^{(1)}\right)(\Lambda)=\left(\hat{I} \circ K^{(2)}\right)(\Lambda)
$$

where $\hat{I} \in \mathcal{B K}_{j}$ is defined by $\hat{I}(V)=I(\hat{V})$, with $\hat{V}=V-Q$ defined by (1.71) in terms of $Q=Q(V, K)$ given by (1.70). The combined effect of these two maps is to transfer the relevant and marginal parts of $K(X)$ for $X \in \mathcal{S}$ into $V$, with the result that $V$ is replaced by $\hat{V}$. The decay in $|X|$ that is encoded in our norms for large sets $X$ by (1.41) allows us to forego any transfer of $K(X)$ for large sets $X$. Map 1 takes advantage of the non-uniqueness of the circle product to replace $K$ by $K^{(1)}$, which results from the transfer of the relevant and marginal parts of $K$ on small sets other than blocks, so that they become concentrated in $K^{(1)}$ on blocks instead. This transfer is achieved using the important change of variables formula given by Proposition 4.1. Then Map 2 transfers the relevant and marginal parts of $K^{(1)}$ that are concentrated on single blocks into $V$ so as to form $\hat{V}$. Thus $\hat{I}$ appears on the right-hand side of (3.5). All three circle products in (3.5) are on scale $j$.

Map 3 is our implementation of the formal power series statement of (1.14) that $\mathbb{E}_{j+1} \theta I_{j}(V, \Lambda) \approx$ $I_{j+1}\left(V_{\mathrm{pt}}, \Lambda\right)$, but now no longer merely as a statement about formal power series. The renormalised polynomial $V_{\mathrm{pt}}$ therefore appears, but since Map 2 has replaced $V$ by $\hat{V}$, we write $V_{\mathrm{pt}}=V_{\mathrm{pt}}(\hat{V})=$ $V_{\mathrm{pt}}(V-Q)$, We define $\tilde{I}_{j+1} \in \mathcal{B K}_{j}($ as in $[20$, (1.23)] $)$ by

$$
\tilde{I}_{j+1}(V, b)=e^{-V(b)}\left(1+W_{j+1}(V, b)\right) \quad\left(b \in \mathcal{B}_{j}\right)
$$

and define $\tilde{I}_{\mathrm{pt}} \in \mathcal{B} \mathcal{K}_{j}$ by

$$
\tilde{I}_{\mathrm{pt}}=\tilde{I}_{j+1}\left(V_{\mathrm{pt}}\right) .
$$

The expectation is performed in Map 3, and $K^{(3)}$ is constructed such that

$$
\mathbb{E}_{+} \theta\left(\hat{I} \circ K^{(2)}\right)(\Lambda)=\left(\tilde{I}_{\mathrm{pt}} \circ K^{(3)}\right)(\Lambda)
$$

The circle product on the left-hand side of (3.8) is at scale $j$, whereas on the right it is at scale $j+$ 1. This entails a slight abuse of notation, in which we regard $\tilde{I}_{\mathrm{pt}}$ in (3.8) as the element of $\mathcal{B} \mathcal{K}_{j+1}$ defined for $B \in \mathcal{B}_{j+1}$ by $\tilde{I}_{\mathrm{pt}}(B)=\prod_{b \in \mathcal{B}_{j}(B)} \tilde{I}_{\mathrm{pt}}(b)$. It is in Map 3 that we change scale in our estimates, with $K^{(2)}$ measured with scale- $j$ norm but $K^{(3)}$ with scale- $(j+1)$ norm. This change of scale is important in revealing the contraction encapsulated in the small parameter $\kappa$ in Theorem 2.2 .

The $K^{(3)}$ produced by Map 3 is larger than what is claimed for $K_{+}$in Theorem 2.2 , due to the fact that it includes perturbative contributions that arise because of the local manner in which we implement the spirit of the proof of (1.14) from [7, Proposition 2.1]. Map 4 reapportions these overly large parts of $K^{(3)}$ by a second application of the change of variables of Proposition 4.1, and thereby constructs a better $K^{(4)}$ such that

$$
\left(\tilde{I}_{\mathrm{pt}} \circ K^{(3)}\right)(\Lambda)=\left(\tilde{I}_{\mathrm{pt}} \circ K^{(4)}\right)(\Lambda) .
$$

Maps 5 and 6 perform three final adjustments, all relatively minor. One adjustment is to put $\tilde{I}_{\mathrm{pt}}$ into the correct scale- $(j+1)$ form of (1.13) rather than as a product over scale- $j$ blocks. The other two deal with the fact that $V_{\mathrm{pt}}$ contains terms $y \tau_{\nabla \nabla}$ and $\delta q \sigma \bar{\sigma}$ which are not present in $V_{+}$. The term $y \tau_{\nabla \nabla}$ is converted to a term $z \tau_{\Delta}$ via summation by parts, at the cost of an adjustment 
to $K$. The term $\delta q \sigma \bar{\sigma}$ is pulled outside the circle product, at the cost of another small adjustment to $K$. This finally leads to

$$
\left(\tilde{I}_{\mathrm{pt}} \circ K^{(4)}\right)(\Lambda)=\left(I_{\mathrm{pt}}^{+} \circ K^{(5)}\right)(\Lambda)=e^{\delta q \sigma \bar{\sigma}}\left(I_{+} \circ K_{+}\right)(\Lambda)
$$

where the precise definition of $I_{\mathrm{pt}}^{+}$is given in Map 5. The circle products in (3.9)-(3.10) are all at scale $j+1$.

The combination of (3.5) and (3.8) $-(3.10)$ gives

$$
\mathbb{E}_{+} \theta(I \circ K)(\Lambda)=e^{\delta q \sigma \bar{\sigma}}\left(I_{+} \circ K_{+}\right)(\Lambda),
$$

which is (1.49). This shows that $\left(V_{+}, K_{+}\right)$preserves the form of the circle product under expectation, as required. To complete the proof of Theorem 2.2(i), it is necessary also to show that there exist $r, M>0$ and $\kappa=O\left(L^{-1}\right)$ such that $K_{+}: \mathcal{D} \times B_{\mathcal{F}}(r \bar{\epsilon}) \rightarrow B_{\mathcal{F}_{+}}(\kappa r \bar{\epsilon})$ with $K_{+}$an analytic map, and such that $\left\|K_{+}(V, 0)\right\|_{\mathcal{F}_{+}} \leq M \bar{\epsilon}^{3}$. These facts are required for each of the norm pairs $\mathcal{F}=\mathcal{F}_{j}(G), \mathcal{F}_{+}=\mathcal{F}_{j+1}\left(T_{0}\right)$ and $\mathcal{F}=\mathcal{F}_{j}(\tilde{G}), \mathcal{F}_{+}=\mathcal{F}_{j+1}(\tilde{G})$. We carry out Maps 1-6 simultaneously for each of the two norm pairs, and prove the estimates and analyticity map by map, culminating in Map 6 with the desired statements for $K_{+}$. Similarly, relevant observations concerning the statements of Theorem 2.2(ii-iv) are made for each Map, and at Map 6 their proof for $K_{+}$is complete. See Section 7 , where the proof of Theorem 2.2 is summed up.

\subsection{Parameters for proof of Theorem 2.2}

For convenience, we gather here the specification of several parameters that occur in the proof of Theorem 2.2.

For each Map $i$ with $i=1,2,4,5,6$ ( $i=3$ is excluded) there is an associated $L$-independent constant $\mu_{i} \geq 1$; the values (typically large) of these constants are determined in Sections 46 . For Map 3, there is an important constant $\kappa_{3}$ which is an $L$-independent multiple of $L^{-1} ; \kappa_{3}$ can be made as small as desired by taking $L$ sufficiently large.

For the constant $r$ that determines the size of balls appearing in Theorem 2.2 , we fix any value

$$
0<r<\min \left\{\frac{1}{\mu_{1} \mu_{2}}, 1\right\} \text {. }
$$

We set $r^{(0)}=r$, and define $r^{(i)}=\mu_{i} r^{(i-1)}$ for $i=1,2,4,5,6$, whereas $r^{(3)}=\kappa_{3} r^{(2)}$. We define the small parameter $\kappa$ by

$$
\kappa=\mu_{1} \mu_{2} \kappa_{3} \mu_{4} \mu_{5} \mu_{6}<1 .
$$

Then $\kappa=\gamma^{*} L^{-1}$ for an $L$-independent constant $\gamma^{*}, r^{(6)}=\kappa r^{(0)}$, and $r^{(i)}<1$ for each $i$.

We fix $a \in\left(0,2^{-d}\right)$ as in the statement of Theorem 2.2. Let $\eta=\eta(d)>1$ be the geometrical constant of Lemma C.3. For $i \in\{0,1,2,3,4,5,6\}$, we fix $a^{(i)}$ such that

$$
0<a^{(2)}<a^{(1)}<a^{(0)}=a<a^{(6)}<a^{(5)}<a^{(4)}<a^{(3)}<\eta a^{(2)} \leq 2^{-d} .
$$

The parameters $a^{(i)}$ determine Banach spaces $\mathcal{F}_{k}^{(i)}=\mathcal{F}_{k}\left(a^{(i)}\right)$ which are defined by replacing $a$ by $a^{(i)}$ in (1.39) and (1.41). 
For $i=1,2$, Map $i$ maps the ball of radius $r^{(i-1)} \bar{\epsilon}$ in $\mathcal{F}_{j}\left(a^{(i-1)}\right)$ into the ball of radius $r^{(i)} \bar{\epsilon}$ in $\mathcal{F}_{j}\left(a^{(i)}\right)$. In Map 3, the scale increases from $j$ to $j+1$, and the ball of radius $r^{(2)} \bar{\epsilon}$ in $\mathcal{F}_{j}\left(a^{(2)}\right)$ is mapped into the ball of radius $r^{(3)} \bar{\epsilon}$ in $\mathcal{F}_{j+1}\left(a^{(3)}\right)$. Map 3 is the beneficial map, as $a^{(3)}$ improves by becoming larger, and also $r^{(3)}$ improves by becoming smaller thanks to the factor $\kappa_{3}$. These improvements undergo degradations in Maps 4-6, in which the ball of radius $r^{(i-1)} \bar{\epsilon}$ in $\mathcal{F}_{j+1}\left(a^{(i-1)}\right)$ is mapped into the ball of radius $r^{(i)} \bar{\epsilon}$ in $\mathcal{F}_{j+1}\left(a^{(i)}\right)$. However the overall effect remains beneficial, with $a^{(6)}>a^{(0)}$, and with $r^{(6)}=\kappa r^{(0)}$ for small $\kappa=\gamma^{*} L^{-1}$. The composition of the six maps is well defined and maps the ball $B_{\mathcal{F}_{j}}\left(r^{(0)} \bar{\epsilon}\right)$ into a small ball $B_{\mathcal{F}_{j}}\left(\kappa r^{(0)} \bar{\epsilon}\right)$.

\subsection{Interaction estimates}

The analysis of the six maps uses estimates on $I, \hat{I}, \tilde{I}_{\mathrm{pt}}$, which we refer to generically as interaction estimates. These estimates, which include stability estimates, rely on the hypothesis that

$$
(V, K) \in \mathcal{D} \times B_{\mathcal{F}}(r \bar{\epsilon})
$$

with $\mathcal{F}$ either $\mathcal{F}(G)$ or $\mathcal{F}(\tilde{G})$, with corresponding choice of $\bar{\epsilon}$ in (1.43). It is the purpose of [20] to provide the interaction estimates, and we appeal frequently to results from [20], some of which we now recall. Further results from [20] are recalled within the analysis of Maps 3,5,6.

The analysis of [20] is missing an ingredient needed here, which is that $K$ now plays a role in interaction estimates. For example, $\hat{I} \in \mathcal{B K}_{j}$, is defined by $\hat{I}(V)=I(\hat{V})$, with $\hat{V}=V-Q$ defined by (1.71) in terms of $Q=Q(V, K)$ given by (1.70) as

$$
Q(B)=\sum_{Y \in \mathcal{S}(\Lambda): Y \supset B} \operatorname{Loc}_{Y, B} I^{-Y} K(Y), \quad B \in \mathcal{B}(\Lambda) .
$$

Similarly, $\tilde{I}_{\mathrm{pt}}=\tilde{I}\left(V_{\mathrm{pt}}(\hat{V})\right)$ depends on $K$ as well as on $V$. The following proposition combines results from [20] with new statements concerning $K$-dependence. For its proof, we recall from (2.1) that $\operatorname{Loc}_{X, Y}$ is a bounded operator, in the sense that for $Y \in \mathcal{P}_{j}$ with $Y \subset X \in \mathcal{S}_{j}$, and for $F^{\prime} \in \mathcal{N}\left(X^{\square}\right)$,

$$
\left\|\operatorname{Loc}_{X, Y} F^{\prime}\right\|_{T_{0}} \prec\left\|F^{\prime}\right\|_{T_{0}} .
$$

We also recall that the enlarged domain $\overline{\mathcal{D}} \supset \mathcal{D}$ is defined in $[20$, (1.85)-(1.86)]; its precise definition does not play a direct role below. Finally, recall that we write $I^{-X}=1 / I(X)$.

Proposition 3.1. Let $I_{*}$ denote any one of $I, \hat{I}, \tilde{I}_{\mathrm{pt}}$, with $j_{*}$ respectively equal to $j, j$, and to either of $j$ or $j+1$. Let $B \in \mathcal{B}_{j}$. Let $(V, K) \in \mathcal{D}_{j} \times B_{\mathcal{F}}\left(r^{(0)} \bar{\epsilon}\right)$. Then $I_{*}(B)$ is an analytic function of $(V, K)$ taking values in $\mathcal{N}\left(B^{\square}\right),\|\cdot\|_{j_{*}}$. In addition, for $F \in \mathcal{N}\left(B^{\square}\right)$ a gauge-invariant polynomial of bounded degree in the fields such that $\pi_{a b} F=0$ when $j \leq j_{a b}$,

$$
\begin{aligned}
\left\|I_{*}(B) F\right\|_{j_{*}, \mathfrak{h}} & \prec\|F\|_{T_{0, j}(\mathfrak{h})}, \\
\left\|I_{*}(B)\right\|_{j_{*}, \mathfrak{h}} & \leq 2 \\
\left\|I_{*}^{-B}\right\|_{T_{0, j_{*}(\mathfrak{h})}} & \leq 2 .
\end{aligned}
$$

In addition, $I^{-B}$ is an analytic function of $V \in \overline{\mathcal{D}}_{j}$ taking values in $\mathcal{N}\left(B^{\square}\right),\|\cdot\|_{T_{0, j}}$. 
Proof. For the case $K=0$, all the above statements are proved already in [20, Propositions 2.2 2.3. In particular, this gives the above statements concerning $I$, since there is no $K$-dependence in I. Our task here is to extend the statements of [20, Propositions 2.2 2.3] to include the dependence of $\hat{V}, V_{\mathrm{pt}}$ on $K \in B_{\mathcal{F}}\left(r^{(0)} \bar{\epsilon}\right)$, as well as on $V$. In fact, the statements of [20, Propositions 2.2 2.3] are all proved in the enlarged domain $\overline{\mathcal{D}} \supset \mathcal{D}$, and this is important below.

We begin with a bound on $Q$, which repeats a step in the proof of Theorem 1.10. Let $(V, K) \in$ $\mathcal{D} \times B_{\mathcal{F}}\left(r^{(0)} \bar{\epsilon}\right)$. We claim that

$$
\|Q(B)\|_{T_{0}} \prec r^{(0)} \bar{\epsilon} .
$$

To prove (3.21), we apply (3.17), together with the $I_{*}=I$ case of (3.18), (3.20), and the product property of the norm, to conclude that if $F \in \mathcal{N}\left(B^{\square}\right)$ then

$$
\left\|I^{X} \operatorname{Loc}_{X, Y} I^{-X} F\right\|_{j} \prec\left\|\operatorname{Loc}_{X, Y} I^{-X} F\right\|_{T_{0, j}} \prec\|F\|_{T_{0, j}} .
$$

With (3.16), this gives

$$
\|Q(B)\|_{T_{0}} \prec \sup _{X \in \mathcal{S}}\|K(X)\|_{T_{0}} \leq\|K\|_{\mathcal{F}} \leq r^{(0)} \bar{\epsilon}
$$

which proves (3.21).

Now that (3.21) has been established, it follows immediately from [20, Proposition 2.4 that $\hat{I}$ obeys the estimates (3.18)-(3.20), that $\hat{I}$ is analytic in $V$ for fixed $Q$, and that $V-Q$ lies in the enlarged domain $\overline{\mathcal{D}^{\prime}}$ (the prime denotes an unimportant change in constants defining $\overline{\mathcal{D}}$ ) on which stability and analyticity of $I$ is proved in [20, Proposition 2.3]. Moreover, it follows from the definition of $Q$ in (3.16) and the results already established for $I$ that $(V, K) \mapsto V-Q$ is analytic as a map from $\mathcal{D} \times B_{\mathcal{F}}\left(r^{(0)} \bar{\epsilon}\right)$ into $\overline{\mathcal{D}}$. Thus $\hat{I}$ has the claimed analyticity. Similarly, since $V-Q \in \overline{\mathcal{D}}$, it follows from [20, Proposition 1.5] that $V_{\mathrm{pt}}(V-Q)$ also lies in the analyticity domain of $I$ both for scale $j$ and scale $j+1$, and hence $\tilde{I}_{\mathrm{pt}}$ is analytic both as a map $V_{\mathrm{pt}} \mapsto \tilde{I}_{\mathrm{pt}}$ defined on $\overline{\mathcal{D}}$, and as a function of $(V, K)$ as desired. This completes the proof.

The proof of analyticity of $I^{-B}$ easily extends to a small $\|V(B)\|_{T_{0}}$-ball, since there is no need for a positivity of the coupling constant $g$ when employing the $T_{0}$ norm; this small extension is used in Theorem 1.10 above. For future reference, we proved in (3.21) that, for $(V, K) \in \mathcal{D} \times B_{\mathcal{F}}\left(r^{(0)} \bar{\epsilon}\right)$,

$$
\|Q(B)\|_{T_{0}} \prec r^{(0)} \bar{\epsilon} .
$$

Also, with the bounds (3.18) and (3.20), the proof of (3.22) extends to show that for any of the three choices of $I_{*}$ above, if $Y \in \mathcal{P}_{j}, Y \subset X \in \mathcal{S}_{j}$ and $F \in \mathcal{N}(X)$ then

$$
\left\|I_{*}^{X} \operatorname{Loc}_{X, Y} I_{*}^{-X} F\right\|_{j} \prec\left\|\operatorname{Loc}_{X, Y} I_{*}^{-X} F\right\|_{T_{0, j}} \prec\|F\|_{T_{0, j}} .
$$

We use (3.25) repeatedly. Note that all norms in (3.25) are at scale $j$, even when $I_{*}=\tilde{I}_{\mathrm{pt}}$.

The following lemma is also useful. Its first two estimates can be understood as a consequence of the fact that $\hat{I}$ and $I$ differ by the contribution of $Q$ to the interaction polynomial, with $Q$ obeying (3.24). The third estimate in the lemma is a reflection of the fact that $\bar{\epsilon}$ provides a measure of the difference between $V$ and $V_{\mathrm{pt}}$. 
Lemma 3.2. Let $(V, K) \in \mathcal{D}_{j} \times B_{\mathcal{F}_{j}}\left(r^{(0)} \bar{\epsilon}\right)$. Let $Q$ be given by (1.70). For $B \in \mathcal{B}_{j}, X \in \mathcal{S}_{j}$ and $F \in \mathcal{N}\left(X^{\square}\right)$,

$$
\begin{gathered}
\|\hat{I}(B)-I(B)\|_{j} \prec \bar{\epsilon}, \\
\|\hat{I}(B)-I(B)(1+Q(B))\|_{T_{0}} \prec_{L} \bar{\epsilon}^{2}, \\
\left\|\operatorname{Loc}_{X}\left(I^{-X}-\tilde{I}_{\mathrm{pt}}^{-X}\right) F\right\|_{T_{0}(\mathfrak{h})} \prec_{L} \bar{\epsilon}\|F\|_{T_{0}(\mathfrak{h})} .
\end{gathered}
$$

Proof. The bounds (3.26)-(3.27) are a consequence of [20, Proposition 2.4], with its hypothesis on $Q$ of provided by (3.24). For (3.28), we write

$$
I^{-X}-\tilde{I}_{\mathrm{pt}}^{-X}=\left(I^{-X}-\hat{I}^{-X}\right)+\left(\hat{I}^{-X}-\tilde{I}_{\mathrm{pt}}^{-X}\right)
$$

and apply the triangle inequality. The second term is estimated in [20, Lemma B.2] (its hypothesis is satisfied since we have established in the proof of Proposition 3.1 that $V-Q \in \overline{\mathcal{D}}$ ). The first term can be estimated similarly, using (3.26), and we omit the details.

The following proposition is used in the proof that $K_{+}$vanishes at weighted infinity.

Proposition 3.3. Let $(V, K) \in \mathcal{D}_{j} \times B_{\mathcal{F}_{j}}\left(r^{(0)} \bar{\epsilon}\right)$, let $B \in \mathcal{B}_{j}$, and let $I_{*}, j_{*}$ denote any of the options in Proposition 3.1. For $F \in \mathcal{N}\left(B^{\square}\right)$ a polynomial of degree at most $p_{\mathcal{N}}$,

$$
\lim _{\|\phi\|_{\Phi_{j}(B \square, \mathfrak{h})} \rightarrow \infty}\left\|I_{*}(B) F\right\|_{T_{\phi, j_{*}}} \mathcal{G}_{j_{*}}^{-1}(X, \phi)=0,
$$

where $\mathcal{G}$ represents $G$ or $\tilde{G}$ according to whether $\mathfrak{h}=\ell$ or $\mathfrak{h}=h$.

Proof. It is proved in [20, (2.8)] that (3.30) holds for $I_{*}=I$ when $V \in \overline{\mathcal{D}}$. We have seen above that $\hat{V}=V-Q \in \overline{\mathcal{D}}_{j}^{\prime}$ and that $V_{\mathrm{pt}}=V_{\mathrm{pt}}(\hat{V}) \in \overline{\mathcal{D}}_{j_{*}}^{\prime}$. The primes represent an unimportant change in constants defining the domain, so the result follows for the other options for $I_{*}$.

\subsection{Analyticity lemmas}

In establishing the analyticity of $K_{+}$, we apply the useful and elementary facts about analytic functions on complex Banach spaces presented in the next lemmas. For a general introduction to analyticity in Banach spaces, see [22,35].

Let $X, Y$ be complex Banach spaces and let $D \subset X$ be an open subset of $X$. Let $L(X, Y)$ denote the space of bounded linear maps from $X$ to $Y$. A map $f: D \rightarrow Y$ is analytic if it is continuously Fréchet differentiable, i.e., if there exists a continuous map $f^{\prime}: D \rightarrow L(X, Y)$ such that

$$
\left\|f(x+\dot{x})-f(x)-f^{\prime}(x) \dot{x}\right\|_{Y}=o\left(\|\dot{x}\|_{Y}\right) \quad \text { as } \dot{x} \rightarrow 0 .
$$

Let $\mathcal{A}$ be an index set. For each $\alpha \in \mathcal{A}$, let $Y_{\alpha}$ be a Banach space and let $w_{\alpha} \geq 1$ be a positive weight. Let $Y=\prod_{\alpha} Y_{\alpha}$ be the weighted product Banach space: an element of $Y$ has the form $y=\left(y_{\alpha} \in Y_{\alpha} \mid \alpha \in \mathcal{A}\right)$ with norm

$$
\|y\|_{Y}=\sup _{\alpha \in \mathcal{A}}\left\|y_{\alpha}\right\|_{Y_{\alpha}} w_{\alpha} .
$$


A collection of maps $f_{\alpha}: D \rightarrow Y_{\alpha}$, for $\alpha \in \mathcal{A}$, naturally determines a map $f: D \rightarrow Y$. In our applications, the weights play a role in estimates but not in proving analyticity, as the following lemma shows.

Lemma 3.4. Let $\mathcal{A}$ be a finite set and let $f=\left(f_{\alpha}\right)$ as above. Then $f: D \rightarrow Y$ is analytic if and only if $f_{\alpha}: D \rightarrow Y_{\alpha}$ is analytic for each $\alpha \in \mathcal{A}$.

Proof. Suppose first that each $f_{\alpha}$ is analytic, and let $f_{\alpha}^{\prime}$ denote its derivative. In particular, $f_{\alpha}^{\prime}: D \rightarrow L(X, Y)$ is continuous. For $x \in D$, let $\hat{f}(x)=\left(f_{\alpha}^{\prime}(x) \mid \alpha \in \mathcal{A}\right)$. Since $\mathcal{A}$ is a finite set, $\hat{f}(x) \in L(X, Y)$ and $x \mapsto \hat{f}(x)$ is a continuous map from $D$ to $L(X, Y)$. Also,

$$
\|f(x+\dot{x})-f(x)-\hat{f}(x) \dot{x}\|_{Y}=\sup _{\alpha \in \mathcal{A}}\left\|f_{\alpha}(x+\dot{x})-f_{\alpha}(x)-f_{\alpha}^{\prime}(x) \dot{x}\right\|_{Y_{\alpha}} \leq \sup _{\alpha \in \mathcal{A}} o\left(\|\dot{x}\|_{Y_{\alpha}}\right) .
$$

Since $\mathcal{A}$ is finite, $\sup _{\alpha \in \mathcal{A}} o\left(\|\dot{x}\|_{Y_{\alpha}}\right) \leq o\left(\|\dot{x}\|_{Y}\right)$, so $f: D \rightarrow Y$ is analytic and $\hat{f}$ is its derivative.

Conversely, suppose that $f$ is analytic. Define $\pi_{\alpha}: Y \rightarrow Y_{\alpha}$ by $\pi_{\alpha} y=y_{\alpha}$. Since $w_{\alpha} \geq 1$ the map $\pi_{\alpha}$ is a bounded linear map from $Y$ to $Y_{\alpha}$, and is therefore analytic. Thus $f_{\alpha}=\pi_{\alpha} \circ f$ is the composition of two analytic maps and hence is also analytic.

Let $n$ be a positive integer. For $i=1,2, \ldots, n$, let $X_{i}$ be a Banach space. Let $Y$ be a Banach space and let $M: X_{1} \times \cdots \times X_{n} \rightarrow Y$ be a multilinear map which is bounded. That is, there is a constant $C_{M}$ such that for any $n$-tuple $x=\left(x_{1}, \ldots, x_{n}\right)$ in $X_{1} \times \cdots \times X_{n}$,

$$
\|M(x)\|_{Y} \leq C_{M} \prod_{i=1}^{n}\left\|x_{i}\right\|_{X_{i}} .
$$

For positive weights $w_{1}, \ldots, w_{n}$ let $X=X_{1} \times \cdots \times X_{n}$ be the Banach space whose norm is

$$
\|x\|_{X}=\sup _{i=1, \ldots, n} w_{i}\left\|x_{i}\right\|_{X_{i}}
$$

Lemma 3.5. The map from $X$ to $Y$ defined by $x \mapsto M(x)$ is analytic with derivative $M^{\prime}(x) \dot{x}=$ $\sum_{i} M\left(x_{1}, \ldots, x_{i-1}, \dot{x}_{i}, x_{i+1}, \ldots, x_{n}\right)$.

Proof. It is straightforward to verify that $\left\|M(x+\dot{x})-M(x)-M^{\prime}(x) \dot{x}\right\|_{Y}$ is bounded by

$$
\left(C_{M} \prod_{i=1}^{n} w_{i}^{-1}\right) \sum_{p=2}^{n}\left(\begin{array}{c}
n \\
p
\end{array}\right)\|x\|_{X}^{n-p}\|\dot{x}\|_{X}^{p}=o\left(\|\dot{x}\|_{X}\right)
$$

as required.

\section{Maps 1-2: Transfer of relevant parts of $K$ to $V$}

We now begin the discussion of the six maps leading from $K$ to $K_{+}$, with Maps $1-2$. In Maps $1-2$, there is no change of scale, and all objects are scale- $j$ objects. To simplify the notation, we do not 
indicate the scale explicitly. The norms appearing in this section are either all the $G$ norm, or all the $\tilde{G}$ norm, each together with its corresponding $\mathcal{F}$ space $\mathcal{F}=\mathcal{F}(G)$ or $\mathcal{F}=\mathcal{F}(\tilde{G})$.

Map 1 (and also Map 4) relies on an essential change of variables formula, which provides a mechanism for rewriting a circle product $(I \circ K)(\Lambda)$ as $\left(I \circ K^{\prime}\right)(\Lambda)$, with $K^{\prime}$ "better" than $K$. The change of variables is given by Proposition 4.1 below. Map 1 uses the change of variables to remove the relevant and marginal parts of $K(X)$ when $X \in \mathcal{S}$ is not a block, by transfering them into $K(B)$ where $B$ is a block. This creates $K^{(1)}$ and leaves $I$ unchanged. Map 2 then removes the relevant and marginal parts of $K(B)$ and transfers them to $V$, thereby creating $\hat{I}$ and $K^{(2)}$.

\subsection{Change of variables}

We work at a fixed scale and do not indicate the scale explicitly in the notation. This section applies for any norm $\|\cdot\|$ on $\mathcal{N}$ which obeys the product property [20, (1.44)].

Let

$$
\mathcal{D}(J)=\{(U, B) \in \mathcal{S} \times \mathcal{B}: U \supset B\} .
$$

Suppose we have a mapping $J: \mathcal{C} \times \mathcal{B} \rightarrow \mathcal{N}$ which obeys, for $U \in \mathcal{C}$ and $B \in \mathcal{B}$, the condition $J(U, B)=0$ if $(U, B) \notin \mathcal{D}(J)$, as well as

$$
\sum_{U:(U, B) \in \mathcal{D}(J)} J(U, B)=0 \quad \text { for fixed } B \in \mathcal{B},
$$

$J(U, B)$ obeys the field locality (in its $B$ argument)

and symmetry conditions of Definition 1.7 .

For example, field locality means that $J(U, B) \in \mathcal{N}\left(B^{\square}\right)$ and Euclidean symmetry means that $E J(U, B)=J(E U, E B)$ for all automorphisms $E$ of $\Lambda$.

Let $\alpha_{I} \geq 1$, and suppose that $I_{\text {in }} \in \mathcal{B K}$ is stable in the sense that

$$
\left\|I_{\text {in }}(B)\right\| \leq \alpha_{I}
$$

For $K_{\text {in }} \in \mathcal{K}$ and $U \in \mathcal{C}$, let

$$
M(U)=K_{\text {in }}(U)-I_{\text {in }}^{U} \sum_{B \in \mathcal{B}(U)} J(U, B) .
$$

Given positive $a_{\text {out }}, a_{\text {in }}, \rho$ we define spaces $\mathcal{F}_{\text {in }}=\mathcal{F}\left(a_{\text {in }}, \rho\right)$ and $\mathcal{F}_{\text {out }}=\mathcal{F}\left(a_{\text {out }}, \rho\right)$, as in (1.41). The open balls of radius $r$ in these spaces are denoted by $B_{\mathcal{F}_{\text {in }}}(r)$ and $B_{\mathcal{F}_{\text {out }}}(r)$.

Proposition 4.1. Let $a_{\mathrm{in}}$ be small as specified in Lemma C.5. Let $a_{\mathrm{out}}<a_{\mathrm{in}}$ and $\left.z^{\prime}\right\rangle \frac{1}{2} z$. Let $\rho$ be sufficiently small depending on the difference $a_{\text {out }}-a_{\text {in }}$. Let $\epsilon \in(0,1)$. Let $J, I_{\text {in }}$ be as specified by (4.1) - (4.4). Suppose that $K_{\mathrm{in}} \in \mathcal{K}$ and $J$ satisfy

$$
\begin{aligned}
& \sup _{\mathcal{D}(J)}\left\|I_{\text {in }}^{U} J(U, B)\right\| \leq \epsilon \rho^{z^{\prime}}, \\
& M \in B_{\mathcal{F}_{\text {in }}}\left(\epsilon \rho^{z}\right) .
\end{aligned}
$$


Then there exists $K_{\text {out }} \in \mathcal{K}$ such that

$$
\begin{aligned}
& \left(I_{\text {in }} \circ K_{\text {out }}\right)(\Lambda)=\left(I_{\text {in }} \circ K_{\text {in }}\right)(\Lambda), \\
& K_{\text {out }} \text { is polynomial in } I_{\text {in }}, \bar{J}, K_{\text {in }}\left(\text { with } \bar{J}(U, B)=I_{\text {in }}^{U} J(U, B)\right), \\
& K_{\text {out }}=M+E \text { with } E \in B_{\mathcal{F}_{\text {out }}}\left(\epsilon \rho^{z+\left(a_{\text {in }}-a_{\text {out }}\right) / 2}\right) .
\end{aligned}
$$

If $K_{\mathrm{in}}=0$ and $J=0$, then $K_{\text {out }}=0$.

The essential conclusion of the Proposition 4.1 is that $K_{\text {out }}$ is both a suitable replacement for $K_{\text {in }}$ by (4.8), and is a small perturbation of $M$ by (4.10). The latter property will be useful in our applications in Maps 1 and 4, where $M$ will have desirable properties. Note also that it follows immediately from (4.7) and (4.10) that

$$
K_{\text {out }} \in B_{\mathcal{F}_{\text {out }}}\left(2 \epsilon \rho^{z}\right) .
$$

We defer the proof of Proposition 4.1 to Appendix D,

\subsection{Map 1: Transfer from small sets to block}

We set $I^{(1)}=I^{(0)}=I$ and $K^{(0)}=K$. Map 1 determines $K^{(1)}$. The structure of the following lemma sets a pattern that we follow throughout our analysis of the six maps. Part (i) is the statement that the output pair $\left(I^{(1)}, K^{(1)}\right)$ is an equivalent representation of the input pair. Part (ii) says that the range of the map is contained in the domain of the next map. Part (iii) finds the image when $K=0$, needed in the proof of (2.10). Part (iv) identifies a property that the map achieves, which did not hold for the input.

As mentioned previously, Map 1 transfers the relevant and marginal parts of $K(X)$ for $X \in$ $\mathcal{S} \backslash \mathcal{B}$ into $K(B)$ where $B \in \mathcal{B}$. Naively, to achieve this we would attempt to replace $K(X)$ by $K(X)-\operatorname{Loc}_{X} K(X)$. However, to maintain stability of the subtracted term, we replace it instead by $I^{X} \operatorname{Loc}_{X} I^{-X} K(X)$, which enjoys the decay properties of the factor $I^{X}$ and will still provide the cancellation we seek. (Recall that we write $I^{-X}=1 / I^{X}$.) Thus, for $X \in \mathcal{S} \backslash B$ we wish to replace $K(X)$ by $K(X)-I^{X} \operatorname{Loc}_{X} I^{-X} K(X)$ via a corresponding adjustment to $K(B)$. This is what Proposition 4.1 permits us to do. To apply Proposition 4.1, we define $J(X, B)=0$ if $(X, B) \notin \mathcal{D}(J)$ and

$$
J(X, B)=\operatorname{Loc}_{X, B} I^{-X} K(X) \text { for } X \in \mathcal{S} \text { with } X \supsetneqq B,
$$

and to achieve the cancellation condition imposed by (4.2), we are forced to define

$$
J(B, B)=-\sum_{Y \in \mathcal{S}: Y \supsetneqq B} \operatorname{Loc}_{Y, B} I^{-Y} K(Y) .
$$

With these definitions, and with $I_{\text {in }}=I$, it then follows from (4.5) and $\sum_{B \subset X} \operatorname{Loc}_{X, B}=\operatorname{Loc}_{X}$ (the latter due to [19, (1.57)]) that

$$
M(X)= \begin{cases}K(X) & X \in \mathcal{C} \backslash \mathcal{S} \\ K(X)-I^{X} \operatorname{Loc}_{X} I^{-X} K(X) & X \in \mathcal{S} \backslash \mathcal{B} \\ K(B)+I^{B} \sum_{Y \in \mathcal{S}, Y \supsetneqq B} \operatorname{Loc}_{Y, B} I^{-Y} K(Y) & X=B \in \mathcal{B} .\end{cases}
$$


The important achievement of (4.14) is the cancellation of the local part of $K(X)$ when $X \in$ $\mathcal{S} \backslash B$. The cost for this cancellation is that these local parts have been transferred to $K(B)$. Recall that $\operatorname{Loc}_{X}=\operatorname{Loc}_{X, X}$ by [19, (1.66)]. It then follows from (4.14) and the definition of $Q(B)$ in (1.70), that

$$
\operatorname{Loc}_{B} I^{-B} M(B)=\sum_{Y \supset B} \operatorname{Loc}_{Y, B} I^{-Y} K(Y)=Q(B)
$$

and this will be cancelled in Map 2 by the $Q$ term in the definition of $\hat{V}$ in (1.71). We now expand on the above by presenting the details for Map 1.

Lemma 4.2. For $u>0$ sufficiently small and $\bar{\epsilon}$ sufficiently small, there exist $\mu_{1} \geq 1$ and $K^{(1)}$ such that, for $(V, K)$ obeying (3.15),

$$
\begin{aligned}
\text { (i) } & (I \circ K)(\Lambda)=\left(I \circ K^{(1)}\right)(\Lambda), \\
\text { (ii) } & K^{(1)} \in B_{\mathcal{F}^{(1)}}\left(r^{(1)} \bar{\epsilon}\right), \\
\text { (iii) } & K^{(1)}=0 \text { when } K=0, \\
\text { (iv) } & \left\|\operatorname{Loc}_{X} I^{-X} K^{(1)}(X)\right\|_{T_{0}} \leq r^{(1)} \bar{\epsilon}^{1+u} \quad X \in \mathcal{S} \backslash \mathcal{B} .
\end{aligned}
$$

Proof. (i) By the hypothesis of Theorem 2.2 that $K \in B_{\mathcal{F}^{(0)}}\left(r^{(0)} \bar{\epsilon}\right)$, and by (3.25), we have

$$
\left\|I^{X} J(X, B)\right\| \prec r^{(0)} \bar{\epsilon}
$$

We choose $\mu_{1}$, depending on the constant in the above inequality, so that with $r^{(1)}=\mu_{1} r^{(0)}$ we can conclude that

$$
\left\|I^{X} J(X, B)\right\| \leq \frac{1}{4} r^{(1)} \bar{\epsilon}^{z^{\prime}}
$$

and also, using (4.14), that

$$
M \in B_{\mathcal{F}^{(0)}}\left(\frac{1}{2} r^{(1)} \bar{\epsilon}\right) .
$$

We apply Proposition 4.1 with $J$ given by (4.12)-(4.13) and with

$$
I_{\text {in }}=I, \quad K_{\text {in }}=K, \quad \rho=\bar{\epsilon}, \quad a_{\text {in }}=a^{(0)}, \quad a_{\text {out }}=a^{(1)}, \quad z=z^{\prime}=1, \quad \epsilon=\frac{1}{2} r^{(1)},
$$

and we define the map $K \mapsto K^{(1)}$ by setting $K^{(1)}=K_{\text {out }}$. Then (i) is an immediate consequence of Proposition 4.1 as soon as we verify that the hypotheses hold. The hypothesis (4.2) holds by construction. The fact that (4.3) holds can be seen from the fact that Loc preserves the relevant symmetries and cannot generate a non-zero constant part (see Lemma F.1 and the proof of Lemma F.2). Hypothesis (4.4) follows from Proposition 3.1, (4.6) follows from (4.17), and (4.7) follows from (4.18).

(ii) The estimate follows from (4.18). For the vanishing at weighted infinity property inherent in the definition of $\mathcal{F}_{+}$(see (1.37) ), the vanishing as $\|\phi\|_{\Phi} \rightarrow \infty$ is a consequence of Proposition 3.3, the definition of $J$ in (4.12)-(4.13), and the fact that $K^{(1)}$ is a polynomial in $I, \bar{J}, K$ by Proposition 4.1. The field locality, symmetry and component factorisation properties follow from the fact that $K^{(1)}=K_{\text {out }} \in \mathcal{K}$ by Proposition 4.1. 
(iii) This is an immediate consequence of Proposition 4.1.

(iv) Let $X \in \mathcal{S} \backslash \mathcal{B}$. By (4.14) and the fact that $\operatorname{Loc}_{X} \circ \operatorname{Loc}_{X}=\operatorname{Loc}_{X}$ by [19, (1.67)],

$$
\operatorname{Loc}_{X} I^{-X} M(X)=0, \quad X \in \mathcal{S} \backslash \mathcal{B} .
$$

Therefore (iv) is equivalent to

$$
\left\|\operatorname{Loc}_{X} I^{-X}\left(K^{(1)}(X)-M(X)\right)\right\|_{T_{0}} \leq r^{(1)} \bar{\epsilon}^{1+u}, \quad X \in \mathcal{S} \backslash \mathcal{B} .
$$

By Proposition 4.1.

$$
K^{(1)}-M \in B_{\mathcal{F}^{(1)}}\left(\frac{1}{2} r^{(1)} \bar{\epsilon}^{1+u}\right) \quad \text { with } \quad u=\frac{1}{2}\left(a^{(0)}-a^{(1)}\right)>0,
$$

and then (4.21) follows from (3.25), where we remove the constant in $\prec$ by decreasing $u$ and taking $\bar{\epsilon}$ small.

We also verify that $K^{(1)}$ obeys the remaining properties of Definition [2.3, namely: $(V, K)$ analyticity, the restriction property, the isometry property, and mass continuity. The isometry property holds because $\iota$ is an algebra homomorphism and, for each polymer $X, K^{(1)}(X)$ is polynomial in $K$. The mass continuity is vacuous here, since $K^{(1)}$ does not depend on the mass.

The restriction property holds in the sense that $K^{(1)}(X)$ is a function of $K(Y)$ for $Y \in X^{\square}$. This follows from the explicit formula for $K_{\text {out }}$ given in (D.13). For example, for the case where $X$ is a single block $B$, (D.14) gives

$$
K^{(1)}(B)=K(B)-J(B, B)+\sum_{U:(U, B) \in \mathcal{D}(J)} I^{U} J(U, B) .
$$

It is due to the dependence of $J$ on $K(U)$ for $U \in B^{\square}$ that $K^{(1)}(B)$ develops its dependence on $K$ in the small set neighbourhood of $B$.

Finally, for the $(V, K)$-analyticity, by Lemma 3.4 it suffices to show that for each polymer $X \in \mathcal{P}$, the map $(V, K) \mapsto K^{(1)}(X)$ is an analytic function from $\mathcal{D} \times B_{\mathcal{F}}\left(r^{(0)} \bar{\epsilon}\right)$ to $\mathcal{N}\left(X^{\square}\right),\|\cdot\|_{j}$ (here $\mathcal{D}$ is the domain for $V$ defined in (1.55), not to be confused with the domain $\mathcal{D}(J)$ for $J$ in Proposition 4.1). We know from Proposition 4.1 that $K^{(1)}(X)$ is a polynomial in $I, \bar{J}, K$. By Lemma 3.5, it suffices to show that each of the maps $(V, K) \mapsto I(B)$ and $(V, K) \mapsto \bar{J}(U, B)$ is an analytic map from $\mathcal{D}$ to $\mathcal{N}\left(B^{\square}\right),\|\cdot\|_{j}$. For $I(B)$, this follows from Proposition 3.1. It therefore suffices to show that the map $(V, K) \mapsto \bar{J}$ is analytic, with $\bar{J}$ defined by (4.9) and (4.12)-(4.13). This map is linear in $K$, Loc is a bounded map on $T_{0}$ by (3.17), and $I^{-X}$ is an analytic map into $\mathcal{N}\left(B^{\square}\right),\|\cdot\|_{T_{0}}$ by Proposition 3.1. Therefore $J(U, B)$ is an analytic function of $(V, K)$ taking values in $\mathcal{V}(B),\|\cdot\|_{T_{0}}$. The bilinear map $(I, J) \mapsto \bar{J}$ is bounded with domain norms $\|\cdot\|_{j},\|\cdot\|_{T_{0}}$ and range norm $\|\cdot\|_{j}$, by Proposition 3.1, and the desired analyticity of $\bar{J}$ then follows from Lemma 3.5.

\subsection{Map 2: Transfer from block to $V$}

Map 2 transfers relevant parts from $K^{(1)}(B)$ into $V$. It provides the rationale for the formula (1.71) for $\hat{V}$. We define

$$
I^{(2)}=\hat{I}=I_{j}(\hat{V}), \quad \delta I^{(2)}=I-\hat{I}, \quad K^{(2)}=K^{(1)} \circ \delta I^{(2)} .
$$


Lemma 4.3. There exists $\mu_{2} \geq 1$ such that for $u>0$ and $\bar{\epsilon}$ both sufficiently small, for $(V, K)$ obeying (3.15),

$$
\begin{aligned}
\text { (i) } & \left(I \circ K^{(1)}\right)(\Lambda)=\left(\hat{I} \circ K^{(2)}\right)(\Lambda), \\
(\text { ii }) & K^{(2)} \in B_{\mathcal{F}^{(2)}}\left(r^{(2)} \bar{\epsilon}\right), \\
\text { (iii) } & K^{(2)}=0 \text { when } K=0, \\
\text { (iv) } & \left\|\operatorname{Loc}_{X} I^{-X} K^{(2)}(X)\right\|_{T_{0}} \leq r^{(2)} \bar{\epsilon}^{1+u} \quad X \in \mathcal{S} .
\end{aligned}
$$

Proof. (i) By Lemma 1.3.

$$
I \circ K^{(1)}=\left(\hat{I}+\delta I^{(2)}\right) \circ K^{(1)}=\left(\hat{I} \circ \delta I^{(2)}\right) \circ K^{(1)}=\hat{I} \circ\left(\delta I^{(2)} \circ K^{(1)}\right)=I^{(2)} \circ K^{(2)} .
$$

(ii) By (3.26) and the $K$ hypothesis of Theorem 2.2 we have $\left\|\delta I^{(2)}(B)\right\|_{j} \prec \bar{\epsilon}$. By choosing $r^{(2)}=\mu_{2} r^{(1)}$ with $\mu_{2}$ sufficiently large, this implies

$$
\delta I^{(2)} \in B_{\mathcal{F}^{(1)}}\left(2^{-2^{d}} r^{(2)} \bar{\epsilon}\right) .
$$

By Lemma 4.2, $K^{(1)} \in B_{\mathcal{F}^{(1)}}\left(2^{-2^{d}} r^{(2)} \bar{\epsilon}\right)$. The desired estimate then follows from Lemma C.1. The fact that $K^{(2)}$ vanishes at weighted infinity is a consequence of the fact that $K^{(1)}$ has this property, and that both $I$ and $\hat{I}$ vanish at weighted infinity by Proposition 3.3 . The field locality, symmetry and component factorisation properties can be verified by inspection.

(iii) For $K=0$, it follows from (1.70)-(1.71) that $\hat{V}=V$. Then (4.24) gives $I^{(2)}=I$ and hence $K^{(2)}=0$.

(iv) We first prove (iv) for the case $X \in \mathcal{S} \backslash \mathcal{B}$. By Lemma 4.2 (iv) (and increasing $\mu_{2}$, it is sufficient to prove the result when $K^{(2)}(X)$ is replaced by $K^{(2)}(X)-K^{(1)}(X)$. By the triangle inequality,

$$
\left\|K^{(2)}(X)-K^{(1)}(X)\right\| \leq \sum_{Y \in \mathcal{P}: Y \varsubsetneqq X}\left\|K^{(1)}(Y)\right\|\left\|\delta I^{(2)}\right\|^{X \backslash Y} .
$$

There are at most $2^{2^{d}}$ terms in this sum. By (4.26) and Lemma 4.2(ii), together with the exclusion of the term $Y=X$ on the right-hand side, we obtain

$$
\left\|K^{(2)}(X)-K^{(1)}(X)\right\| \prec\left(r^{(2)}\right)^{2} \bar{\epsilon}^{2} .
$$

Then we obtain the desired bound by using (3.25), $r^{(2)}<1, u<1$, and choosing $\bar{\epsilon}$ small.

Finally, we prove (iv) for the case $X=B \in \mathcal{B}$. By definition, $K^{(2)}(B)=K^{(1)}(B)+\delta I^{(2)}(B)$. Therefore, by (4.15),

$$
\begin{aligned}
\operatorname{Loc}_{B} I^{-B} K^{(2)}(B) & =\operatorname{Loc}_{B} I^{-B} K^{(1)}(B)+\operatorname{Loc}_{B} I^{-B} \delta I^{(2)}(B) \\
& =\operatorname{Loc}_{B} I^{-B}\left(K^{(1)}(B)-M(B)\right)+\operatorname{Loc}_{B}\left[I^{-B} \delta I^{(2)}(B)+Q(B)\right],
\end{aligned}
$$

where we used the fact that $\operatorname{Loc}_{B} Q(B)=Q(B)$, which is a consequence of the fact that $\operatorname{Loc}_{X} \circ$ $\operatorname{Loc}_{X}=\operatorname{Loc}_{X}$ by [19, (1.67)]. By (3.20), (3.17), (3.25), (3.27), and (4.22), it follows that

$$
\left\|\operatorname{Loc}_{B} I^{-B} K^{(2)}(B)\right\|_{T_{0}} \leq r^{(2)} \bar{\epsilon}^{1+u}
$$

as required. 
We also verify that $K^{(2)}$ obeys the $(V, K)$-analyticity property, the restriction property, the isometry property, and mass continuity. The restriction property is evident from the definition of $K^{(2)}$, and the mass continuity is again vacuous.

From the above construction and the explicit formula (D.13) for the change of variables in Appendix Dit follows that, for each polymer $X, K^{(2)}(X)$ is a polynomial in $\hat{I}$ and $K^{(1)}$. Since $\iota$ is a homomorphism the isometry property holds provided for each block $B$ the function $\hat{I}\left(K^{(1)}, B\right)$ of $\left.K^{(1)}\right|_{B}$ satisfies $\iota \hat{I}\left(K^{(1)}, B\right)=\hat{I}\left(\iota K^{(1)}, \iota B\right)$. We omit this mechanical step.

For the analyticity, we observe that $K^{(2)}$ is multilinear in $I, \hat{I}, K^{(1)}$. We have already verified the analyticity of $K^{(1)}$ in the previous section, and the analyticity of $I$ and $\hat{I}$ is given by Proposition 3.1 . The desired analyticity of $K^{(2)}$ then follows from Lemmas 3.4 3.5.

\section{Maps 3-4: Expectation and change of scale}

Map 3 expresses the action of the expectation $\mathbb{E}_{+}$in terms of $K \circ I$. A reblocking takes place in the process, yielding $K^{(3)} \in \mathcal{K}_{j+1}$. Thus we measure the size of $K^{(3)}$ in scale $j+1$ norms-the change of norm is an important ingredient in establishing contractivity.

In Section 5.1, we define $I^{(3)}$ and $K^{(3)}$ and summarise the principal facts about Map 3. The proof of estimates on $K^{(3)}$ is deferred to Section [5.2, which relies heavily on results from [20] that were designed expressly for this analysis.

There are two types of potentially dangerous contributions to $K^{(3)}$. One type consists of the leading contributions in $K^{(3)}$ which form a part of the perturbative contributions that arise in $K^{(3)}$ even when $K^{(2)}=0$. These perturbative contributions are larger than what is permitted in $K_{+}$ when $K=0$. The second type consists of the contribution to $K^{(3)}$ which is linear in $K^{(2)}$. The latter contribution will be shown to be contractive due to our having removed the relevant and marginal parts of $K^{(2)}$ in Map 2, as expressed by Lemma 4.3(iv); large $L$ plays an important role in this step. The leading perturbative contributions will be redistributed by Map 4, via a second application of the change of variables implemented by Proposition 4.1. leading to $K^{(4)}$ which obeys the better estimate of Lemma 5.8(iii), which is the principal achievement of this section.

\subsection{Map 3: Expansion, expectation, change of scale}

The next proposition gives a formula for $K^{(3)}$. This is the only place where the factorisation property (1.12) of $\mathbb{E}_{+}$is used: it ensures that $K^{(3)}$ obeys the component factorisation property demanded by the space $\mathcal{K}_{j+1}$ in Definition 1.7 .

Recall that $\tilde{I}$ was defined in (3.6). We set

$$
I^{(3)}=\tilde{I}_{j+1}\left(V_{\mathrm{pt}}\right)=\tilde{I}_{\mathrm{pt}},
$$

and define $\delta I \in \mathcal{B K}_{j}$ (the space $\mathcal{B K}_{j}$ is augmented here by the fluctuation fields introduced by $\theta$ ) by

$$
\delta I=\theta I^{(2)}-I^{(3)}=\theta \hat{I}-\tilde{I}_{\mathrm{pt}}
$$

Thus, for $U \in \mathcal{P}_{j}$,

$$
\delta I^{U}=\prod_{b \in \mathcal{B}_{j}(U)}\left(\theta \hat{I}(b)-\tilde{I}_{\mathrm{pt}}(b)\right) .
$$


Proposition 5.1. Given $K^{(2)} \in \mathcal{K}_{j}$,

$$
\mathbb{E}_{+} \theta\left(\hat{I} \circ K^{(2)}\right)(\Lambda)=\left(\tilde{I}_{\mathrm{pt}} \circ K^{(3)}\right)(\Lambda),
$$

where, for $U \in \mathcal{P}_{j+1}$,

$$
K^{(3)}(U)=\sum_{X \in \overline{\mathcal{P}}_{j}(U)} \tilde{I}_{\mathrm{pt}}^{U \backslash X} \mathbb{E}_{+}\left(\delta I \circ \theta K^{(2)}\right)(X)
$$

Also, $K^{(3)} \in \mathcal{F}_{j+1}$.

Proof. We use $\theta \hat{I}(B)=\tilde{I}_{\mathrm{pt}}(B)+\delta I(B)$ and Lemma 1.3 to see that

$$
\mathbb{E}_{+} \theta\left(\hat{I} \circ K^{(2)}\right)(\Lambda)=\mathbb{E}_{+}\left(\tilde{I}_{\mathrm{pt}} \circ \delta I \circ \theta K^{(2)}\right)(\Lambda) .
$$

There is no $\theta$ operating on $\tilde{I}_{\mathrm{pt}}$, and this factor contains no fluctuation fields upon which $\mathbb{E}_{+}$can act. Therefore, with sums over disjoint $X_{K}, X_{\delta I}$, we have

$$
\begin{aligned}
\mathbb{E}_{+} \theta\left(\hat{I} \circ K^{(2)}\right)(\Lambda) & =\sum_{X_{K}, X_{\delta I} \in \mathcal{P}_{j}(\Lambda)} \mathbb{E}_{+}\left(\delta I^{X_{\delta I}} \theta K^{(2)}\left(X_{K}\right)\right) \tilde{I}_{\mathrm{pt}}^{\Lambda \backslash\left(X_{K} \cup X_{\delta I}\right)} \\
& =\sum_{X_{K}, X_{\delta I} \in \mathcal{P}_{j}(\Lambda)} \mathbb{E}_{+}\left(\delta I^{X_{\delta I}} \theta K^{(2)}\left(X_{K}\right)\right) \tilde{I}_{\mathrm{pt}}^{\bar{X}_{K} \cup X_{\delta I} \backslash\left(X_{K} \cup X_{\delta I}\right)} \tilde{I}_{\mathrm{pt}}^{\Lambda \backslash \overline{X_{K} \cup X_{\delta I}}} .
\end{aligned}
$$

We write $X_{I}=\overline{X_{K} \cup X_{\delta I}} \backslash\left(X_{K} \cup X_{\delta I}\right)$ and $U=X_{K} \cup X_{\delta I} \cup X_{I}=\overline{X_{K} \cup X_{\delta I}}$, to obtain

$$
\mathbb{E}_{+} \theta\left(\hat{I} \circ K^{(2)}\right)(\Lambda)=\sum_{X_{K}, X_{\delta I}, X_{I} \in \mathcal{P}_{j}(\Lambda)} \mathbb{E}_{+}\left(\delta I^{X_{\delta I}} \theta K^{(2)}\left(X_{K}\right)\right) \tilde{I}_{\mathrm{pt}}^{X_{I}} \tilde{I}_{\mathrm{pt}}^{\Lambda \backslash U}
$$

where the sum is over disjoint $X_{K}, X_{\delta I}, X_{I}$ with $\overline{X_{K} \cup X_{\delta I}}=X_{K} \cup X_{\delta I} \cup X_{I}=U$ (see Figure 21). With $K^{(3)}$ defined as in (5.5) this becomes the conclusion (5.4).

To see that $K^{(3)}$ is in $\mathcal{K}_{j+1}$, the field locality is straightforward. Component factorisation is an immediate consequence of the factorisation properties for $K^{(2)}$ and the finite-range property (1.12) of $\mathbb{E}_{+}$, and the symmetry properties required by Definition 1.7 follow for $K^{(3)}$ from Lemma F.3.

Finally, we show that $K^{(3)}$ vanishes at weighted infinity, as required by the definition of the space $\mathcal{F}^{(3)}$. For this, we rewrite (5.5) as

$$
K^{(3)}(U)=\sum_{X \in \overline{\mathcal{P}}_{j}(U)} \tilde{I}_{\mathrm{pt}}^{U \backslash X} \mathbb{E}_{+} \theta\left(\hat{I}^{X} K^{(2)}(X)\right)-\sum_{X \in \overline{\mathcal{P}}_{j}(U)} \tilde{I}_{\mathrm{pt}}^{U} \mathbb{E}_{+} \theta K^{(2)}(X) .
$$

By Proposition 3.3, each of $\tilde{I}_{\mathrm{pt}}^{U}, \tilde{I}_{\mathrm{pt}}^{U \backslash X}, \hat{I}^{X}$ vanishes as $\|\phi\|_{\Phi} \rightarrow \infty$. So does $K^{(2)}(X)$, by Lemma 4.3, By Proposition B.7, the property of vanishing at weighted infinity is preserved by the operator $\mathbb{E}_{+} \theta$, and the proof is complete.

For connected sets $U \in \mathcal{C}_{j+1}$, we define

$$
\begin{aligned}
& h(U)=\sum_{X \in \overline{\mathcal{P}}_{j}(U)} \tilde{I}_{\mathrm{pt}}^{-X} \mathbb{E}_{j+1} \delta I^{X}, \\
& k(U)=\sum_{X \in \overline{\mathcal{C}}_{j}(U)} \tilde{I}_{\mathrm{pt}}^{-X} \mathbb{E}_{j+1} \theta K^{(2)}(X),
\end{aligned}
$$




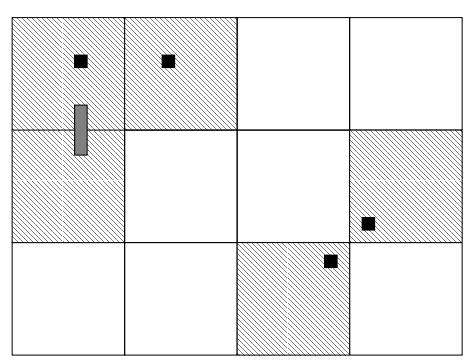

Figure 2: The black squares represent $X_{\delta I}$, the small shaded polymer represents $X_{K}$ and the five large shaded squares comprise $X_{I}$ in (5.8).

where $\overline{\mathcal{P}}_{j}(U)$ is defined in Definition 1.4 and

$$
\overline{\mathcal{C}}_{j}(U)=\left\{X \in \mathcal{C}_{j} \mid \bar{X}=U\right\} .
$$

Then we rewrite the formula (5.5) for $K^{(3)}$ as

$$
K^{(3)}=\tilde{I}_{\mathrm{pt}} h+\tilde{I}_{\mathrm{pt}} k+R .
$$

Here $\tilde{I}_{\mathrm{pt}} h$ is the perturbative contribution resulting from the terms in (5.5) in which no explicit $K^{(2)}$ appears, $\tilde{I}_{\mathrm{pt}} k$ is the contribution to (5.5) that contains exactly one factor $K^{(2)}$ supported on a connected set, and $R$ consists of the remaining terms in $K^{(3)}$ which are not included in $\tilde{I}_{\mathrm{pt}} h+\tilde{I}_{\mathrm{pt}} k$. In Section 5.2 below (see (5.31) and Lemmas 5.6 5.7), we prove that there exist $\kappa^{*}$ and $u>0$ such that for all $U \in \mathcal{P}_{j+1}$,

$$
\begin{aligned}
\left\|\tilde{I}_{\mathrm{pt}}(U) h(U)\right\|_{j+1} & \prec_{L} \bar{\epsilon}^{2+f_{j+1}\left(a^{(3)}, U\right)} \\
\left\|\tilde{I}_{\mathrm{pt}}(U) k(U)\right\|_{j+1} & \leq \kappa^{*} r^{(2)} \bar{\epsilon}^{1+f_{j+1}\left(a^{(3)}, U\right)} \\
\|R(U)\|_{j+1} & \prec_{L} \bar{\epsilon}^{1+u+f_{j+1}\left(a^{(3)}, U\right)}
\end{aligned}
$$

Crucially, $\kappa^{*}$ can be made as small as desired by taking $L$ sufficiently large. In fact, as we shall see, $\kappa^{*}$ is an $L$-independent multiple of $L^{-1}$.

The following lemma provides a summary of Map 3. Parts (i-ii) identify what this map achieves - it performs the expectation with the resulting $K^{(3)}$ properly bounded at scale $j+1$. There are two important improvements in the lemma: the radius of the ball for $K^{(3)}$ has decreased by the factor $\kappa_{3}$ compared to the ball for $K^{(2)}$, and the value of $a^{(3)}$ has increased as in (3.14).

Lemma 5.2. Let $\kappa_{3}=2 \kappa^{*}$. For $L$ sufficiently large and $\bar{\epsilon}$ sufficiently small depending on $L$, there exists $K^{(3)}$ such that, for $(V, K)$ obeying (3.15),

$$
\begin{aligned}
(i) & \mathbb{E}_{+}\left(\hat{I} \circ K^{(2)}\right)(\Lambda)=\left(\tilde{I}_{\mathrm{pt}} \circ K^{(3)}\right)(\Lambda), \\
(i i) & K^{(3)} \in B_{\mathcal{F}^{(3)}}\left(r^{(3)} \bar{\epsilon}\right), \\
(\text { iii }) & K^{(3)}=\tilde{I}_{\mathrm{pt}} h\left(\text { with } V_{\mathrm{pt}}(\hat{V})=V_{\mathrm{pt}}(V)\right) \text { if } K=0 .
\end{aligned}
$$

Proof. (i) This follows immediately from Proposition 5.1 .

(ii) A decomposition of $K^{(3)}$ into three terms is given in (5.13). According to (5.14) $-(5.16)$, once 
we set $\kappa_{3}=2 \kappa^{*}$ and choose $\bar{\epsilon}$ to be sufficiently small depending on $L$, we find that $K^{(3)}$ obeys the estimate implied by the statement that $K^{(3)} \in B_{\mathcal{F}^{(3)}}\left(\kappa_{3} r^{(2)} \bar{\epsilon}\right)$. The fact that $K^{(3)}$ has the properties required of membership in the space $\mathcal{F}^{(3)}$ was established already in Proposition 5.1.

(iii) This follows from (5.13) and the fact that $K^{(2)}=0$ if $K=0$ by Lemma 4.3)(iii), together with the fact that $\hat{V}=V$ when $K=0$ by (1.71).

The restriction and isometry properties of Definition 2.3 can be verified from the definition of $K^{(3)}$ as in maps 1,2 . For the mass continuity and $(V, K)$-analyticity, we argue as follows.

Mass continuity of Map 3. The mass continuity is needed in the setting of Theorem 1.13. At this stage of the proof, we consider continuity of $m^{2} \mapsto K^{(3)}(U)$ for each polymer $U \in \mathcal{P}_{j+1}$, as a map into $\mathcal{N}\left(U^{\square}\right)$ with either norm $\|\cdot\|_{j}$ or $\|\cdot\|_{j+1}$. (Further discussion occurs in Section 7 ,) The dependence of $K^{(3)}(U)$ on the mass $m$ in (5.9) arises from the mass dependence of the covariance $C_{j+1}$ in the decomposition of $\left(-\Delta+m^{2}\right)^{-1}$, and occurs in in two ways.

One occurrence is via the dependence of $V_{\mathrm{pt}}$, and hence of $I^{(3)}=\tilde{I}_{\mathrm{pt}}$, on $C_{j+1}$ and thus on the mass. The continuity of the coefficients of $V_{\mathrm{pt}}$ in small non-negative $\mathrm{m}^{2}$ is established in $[\underline{8}$, Proposition 4.4. With the analyticity of $I$ in $V_{\mathrm{pt}}$ given by Proposition [3.1, the continuity of $\tilde{I}_{\mathrm{pt}}^{X}$ (as an element of $\left.\mathcal{N}\left(X^{\square}\right),\|\cdot\|_{j+1}\right)$ in $m$ follows.

The second occurrence is in the covariance $C_{j+1}$ of the expectation in the definition of $K^{(3)}$ in (5.5). According to Proposition $\mathbb{B} .2$, given $F(X) \in \mathcal{N}\left(X^{\square}\right)$, the linear map $m^{2} \mapsto \mathbb{E}_{j+1} \theta F(X)$ from the interval $\mathbb{I}_{j+1}$ of $(1.52)$ to $\mathcal{N}\left(X^{\square}\right),\|\cdot\|_{j+1}$ is a continuous function. Therefore $K^{(3)}(U)$ is a continuous function of $m^{2} \in \mathbb{I}_{j+1}$.

Analyticity of Map 3. By Proposition 3.1, for $b \in \mathcal{B}_{j}$, the map $(V, K) \mapsto \hat{I}(b)$ is an analytic map into $\mathcal{N}\left(b^{\square}\right),\|\cdot\|_{j}$, and the map $(V, K) \mapsto \tilde{I}_{\mathrm{pt}}(b)$ is an analytic map into $\mathcal{N}\left(b^{\square}\right),\|\cdot\|_{j+1}$. In Section 4.3, it is shown that the map $(V, K) \mapsto K^{(2)}(X)$ is also analytic into $\mathcal{N}\left(X^{\square}\right),\|\cdot\|_{j}$. By Proposition 5.3 below, the map $\mathbb{E}_{+} \theta$ is a bounded linear map from $\mathcal{N}\left(X^{\square}\right),\|\cdot\|_{j}$ to $\mathcal{N}\left(X^{\square}\right),\|\cdot\|_{j+1}$. Therefore, the formula (5.9) expresses $K^{(3)}(U)$ as a finite sum of bounded multilinear maps evaluated on factors which are themselves analytic in $(V, K)$. By Lemmas 3.4 3.5, $(V, K) \mapsto K^{(3)}$ is an analytic map into $\mathcal{F}^{(3)}$.

\subsection{Map 3: Estimates}

Throughout this section, we work exclusively with the norm pairs (1.35)-(1.36). Norms with subscripts are used to denote these pairs of norms: when the scale- $j$ norm is the $G_{j}$ norm then the scale- $(j+1)$ norm is the $T_{0, j+1}$ norm, and when the scale- $j$ norm is the $\tilde{G}_{j}$ norm then the scale$(j+1)$ norm is the $\tilde{G}_{j+1}$ norm. All the estimates given in terms of norms $\|\cdot\|_{j}$ and $\|\cdot\|_{j+1}$ apply for each of these two choices of norm pairs. Our goal is to prove (5.14)-(5.16). We begin with the bound on $\tilde{I}_{\mathrm{pt}} h$. For this, we recall the following integration property, which is [20, Proposition 2.7].

Proposition 5.3. Let $\bar{\epsilon}$ be sufficiently small (depending on L). There is an $\alpha_{\mathbb{E}}>0$ (independent of $L$ ) such that for disjoint $X, Y \in \mathcal{P}_{j}$ and for $F(Y) \in \mathcal{N}\left(Y^{\square}\right)$,

$$
\left\|\mathbb{E}_{j+1} \delta I^{X} \theta F(Y)\right\|_{j+1} \leq \alpha_{\mathbb{E}}^{|X|_{j}+|Y|_{j}} \bar{\epsilon}^{|X|_{j}}\|F(Y)\|_{j}
$$


where the pair of norms is given by either choice of (1.35) or (1.36), and where $|X|_{j}$ denotes the number of scale- $j$ blocks in $X$.

The following proposition is overkill for our needs in Map 3, but we will use its full power in Map 4 and it is convenient to state it here in this form. The leading part of $h$, denoted $h_{\text {lead, }}$ was defined in [20, (2.19)] by

$$
h_{\text {lead }}(U, B)= \begin{cases}-\frac{1}{2} \mathbb{E}_{\pi, j+1} \theta(V(B) ; V(\Lambda \backslash B)) & U=B \\ \frac{1}{2} \mathbb{E}_{\pi, j+1} \theta(V(B) ; V(U \backslash B)) & U \supset B,|U|_{j+1}=2 \\ 0 & \text { otherwise. }\end{cases}
$$

The subscript $\pi$ above corresponds to a bookkeeping device (see [20, (2.18)]) that does not play an explicit role in what follows. It is shown in [20, $(2.22)]$ that, given $B \in \mathcal{B}_{j+1}$,

$$
\sum_{U:(U, B) \in \mathcal{D}\left(h_{\text {lead }}\right)} h_{\text {lead }}(U, B)=0,
$$

and this property is essential in Map 4 where it is used in conjunction with (4.2). We define

$$
h_{\text {lead }}(U)=\sum_{B \in \mathcal{B}(U)} h_{\text {lead }}(U, B) .
$$

We now extend the definition of $f_{j}(a, X)$ in (1.39) as follows. Given $z \geq 0$, for $a \in\left(0,2^{-d}\right]$ and $X \in \mathcal{P}_{j}$ we define

$$
f_{j}(z, a, X)= \begin{cases}z+f_{j}(a, X) & X \neq \varnothing \\ 0 & X=\varnothing\end{cases}
$$

Proposition 5.4. There exists a constant $c_{\text {lead }}=c_{\text {lead }}(L)$ such that

$$
\left\|\tilde{I}_{\mathrm{pt}}(U) h_{\text {lead }}(U, B)\right\|_{j+1} \leq c_{\text {lead }} \bar{\epsilon}^{z_{\text {lead }}}, \quad z_{\text {lead }}=2 .
$$

Also, for $U \in \mathcal{C}_{j+1}$,

$$
\left\|\tilde{I}_{\mathrm{pt}}(U)\left[h(U)-h_{\text {lead }}(U)\right]\right\|_{j+1} \leq c_{\mathrm{pt}} \bar{\epsilon}^{f_{j+1}\left(z_{h}, a^{(3)}, U\right)}, \quad z_{h}=3 .
$$

The constants $c_{\mathrm{lead}}, c_{\mathrm{pt}}$ may depend on $L$, and the norms are either of (1.35) or (1.36).

Proof. The inequality (5.22) is proved in [20, Proposition 2.5]. For (5.23), given $U \in \mathcal{C}_{j+1}$, we define

$$
\begin{aligned}
h_{\mathrm{red}}(U) & =\sum_{X \in \overline{\mathcal{P}}_{j}(U):|X|_{j} \leq 2} \tilde{I}_{\mathrm{pt}}^{-X} \mathbb{E}_{+} \delta I^{X}, \\
h_{\mathrm{rem}}(U) & =\sum_{X \in \overline{\mathcal{P}}_{j}(U):|X|_{j} \geq 3} \tilde{I}_{\mathrm{pt}}^{-X} \mathbb{E}_{+} \delta I^{X},
\end{aligned}
$$

so that

$$
h(U)=h_{\mathrm{red}}(U)+h_{\mathrm{rem}}(U) .
$$


The bound (5.23) with $h$ replaced by $h_{\text {red }}$ was proved in [20, Proposition 2.6], and hence it suffices to prove that, for $U \in \mathcal{C}_{j+1}$,

$$
\left\|\tilde{I}_{\mathrm{pt}}^{U} h_{\mathrm{rem}}(U)\right\|_{j+1} \leq c_{\mathrm{pt}} \bar{\epsilon}^{f_{j+1}\left(z_{h}, a, U\right)}
$$

But by definition, the fact that $|\bar{X}|_{j+1} \leq|X|_{j}$, and Proposition 5.3,

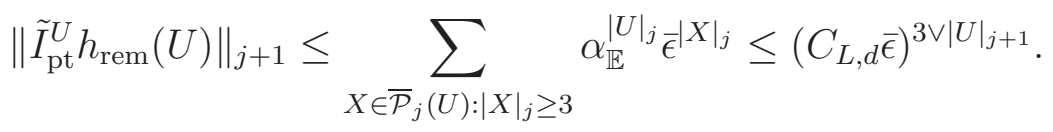

If $|U|_{j+1} \leq 2^{d}$ then the right-hand side is less than $C_{L, d}^{3} \bar{\epsilon}^{3}$, and (5.27) holds in this case. On the other hand, if $|U|_{j+1} \geq 2^{d}+1$, then, since $3 \leq 2^{d}$,

$$
|U|_{j+1} \geq 3+\left(|U|_{j+1}-2^{d}\right)=3+a\left(|U|_{j+1}-2^{d}\right)+(1-a)\left(|U|_{j+1}-2^{d}\right),
$$

where we take $a=a^{(3)}$ (though in fact any larger $a<1$ would also work here). Thus we can choose $t>0$ depending on $a$ such that

$$
|U|_{j+1} \geq 3+\left(|U|_{j+1}-2^{d}\right)=3+a\left(|U|_{j+1}-2^{d}\right)+t|U|_{j+1}
$$

The resulting factor $\bar{\epsilon}^{t|U|_{j+1}}$ can be used to control $C_{L, d}^{|U|_{j+1}}$ and the desired result follows.

Since $U$ is a small set, $\bar{\epsilon}^{f_{j+1}\left(z_{h}, a^{(3)}, U\right)}=\bar{\epsilon}^{z_{h}}$ is much smaller than $\bar{\epsilon}^{f_{j+1}\left(z_{\text {lead }}, a^{(3)}, U\right)}=\bar{\epsilon}^{z_{\text {lead }}}$. Therefore, it is an immediate consequence of Proposition 5.4, via the triangle inequality, that

$$
\left\|\tilde{I}_{\mathrm{pt}}(U) h(U)\right\|_{j+1} \leq c_{\mathrm{pt}} \bar{\epsilon}^{f_{j+1}\left(z_{\mathrm{lead}}, a^{(3)}, U\right)} .
$$

This proves (5.14). The constants $c_{\mathrm{pt}}$ in (5.31) and (5.23) may be larger than the constant with the same name in [20, Proposition 2.6], but this is of no importance. The inequality (5.23) shows that $h_{\text {lead }}(U)$ is in fact the leading part of $h(U)$.

Next we estimate the term $\tilde{I}_{\mathrm{pt}} k$ and prove (5.15). For this we will apply the following crucial estimate, which is [20, Proposition 2.8]. Given $X \subset \Lambda$, we define

$$
\gamma=\gamma(X)=L^{-d-1}+L^{-1} \mathbb{1}_{X \cap\{a, b\} \neq \varnothing .}
$$

Proposition 5.5. Let $X \in \mathcal{S}_{j}$ and $U=\bar{X}$. Let $F(X) \in \mathcal{N}\left(X^{\square}\right)$ be such that $\pi_{\alpha} F(X)=0$ when $X(\alpha)=\varnothing$, and such that $\pi_{a b} F(X)=0$ unless $j \geq j_{a b}$ (recall (1.28) ). Let $\kappa_{F}=\|F(X)\|_{j}$ and let $\kappa_{\text {Loc } F}=\left\|\tilde{I}^{X} \operatorname{Loc}_{X} \tilde{I}^{-X} F(X)\right\|_{j}$. Then

$$
\left\|\tilde{I}^{U \backslash X} \mathbb{E}_{+} \theta F(X)\right\|_{j+1} \prec \gamma(X) \kappa_{F}+\kappa_{\mathrm{Loc} F},
$$

where the pair of norms is given by either choice of (1.35) or (1.36).

The following result is at the heart of our method. It establishes the contractivity of the linear part of the map $K^{(2)} \mapsto K^{(3)}$ via two different and essential principles: for small sets $X$ we have arranged in Lemma 4.3(iii) that $K^{(2)}$ has a small relevant/marginal local part and we can apply Proposition 5.5, while for large sets we apply the geometric fact in Lemma C.3 to exploit the decay of $K(X)$ in the size of $X$. 
Lemma 5.6. For $L$ sufficiently large, $\bar{\epsilon}$ sufficiently small depending on $L$, and $U \in \mathcal{C}_{j+1}$,

$$
\left\|\tilde{I}_{\mathrm{pt}}(U) k(U)\right\|_{j+1} \leq \kappa^{*} r^{(2)} \bar{\epsilon}^{1+f_{j+1}\left(a^{(3)}, U\right)}
$$

where $\kappa^{*}$ is an L-independent multiple of $L^{-1}$.

Proof. By Lemma 4.3,

$$
\left\|K^{(2)}(X)\right\|_{j} \leq r^{(2)} \bar{\epsilon}
$$

In the definition of $k(U)$ in (5.11), we first consider those terms in the sum over $X$ where $X \in \mathcal{S}_{j}$ and we prove that the contribution from these terms is bounded by the right-hand side of (5.34). By Lemma 4.3 and (3.28) (reducing $u$ slightly to absorb the $L$-dependence in (3.28)),

$$
\left\|\operatorname{Loc}_{X} \tilde{I}_{\mathrm{pt}}^{-X} K^{(2)}(X)\right\|_{T_{0}} \prec r^{(2)} \bar{\epsilon}^{1+u}
$$

so it follows from the first inequality of (3.25) that

$$
\left\|\tilde{I}_{\mathrm{pt}}^{X} \operatorname{Loc}_{X} \tilde{I}_{\mathrm{pt}}^{-X} K^{(2)}(X)\right\|_{j} \prec r^{(2)} \bar{\epsilon}^{1+u} .
$$

We have already shown in Proposition 1.14 that $\pi_{a b} V_{\mathrm{pt}}=0$ unless $j \geq j_{a b}$. That proof used the observation that no small set can contain both points $a, b$ when $j<j_{a b}$. By taking $L$ larger if necessary, it is similarly the case that the small set neighbourhood $X^{\square}$ of a small set $X$ cannot contain $a, b$ when $j<j_{a b}$. By the assumption in Definition 1.7 that $\pi_{a b} K(X)=0$ unless $a, b \in X^{\square}$, and by the definition of $K^{(2)}$, we conclude that $\pi_{a b} K^{(2)}(X)=0$ when $j<j_{a b}$. It then follows from Proposition 5.5 that

$$
\left\|\tilde{I}_{\mathrm{pt}}^{U \backslash X} \mathbb{E}_{+} \theta K^{(2)}(X)\right\|_{j+1} \prec \gamma(X) r^{(2)} \bar{\epsilon}+r^{(2)} \bar{\epsilon}^{1+u}
$$

We drop the second term because it is small compared with the first term. After summation over the $O\left(L^{d}\right)$ small sets whose closure is $U$, and by taking $\bar{\epsilon}$ small depending on $L$, the resulting right-hand side is bounded above by a multiple of $L^{-1} r^{(2)} \bar{\epsilon}$, which is the correct size for (5.34). This completes the analysis for $X \in \mathcal{S}_{j}$.

We now consider those terms in the sum over $X$ in (5.11) due to $X \notin \mathcal{S}_{j}$, and prove that the contribution from these terms is bounded by the right-hand side of (5.34). In this sum over $X$ there are fewer than $2^{|U|_{j}}$ terms. Therefore, by Proposition 3.1. (5.17), (5.35), and Lemma C.3, there is a constant $C=C(d, L)$ such that

$$
\begin{aligned}
\left\|\sum_{X \in \overline{\mathcal{C}}_{j}(U): X \notin \mathcal{S}_{j}} \tilde{I}_{\mathrm{pt}}^{U \backslash X} \mathbb{E}_{j+1} \theta K^{(2)}(X)\right\|_{j+1} & \leq r^{(2)} C^{|U|_{j}} \sup _{X \in \overline{\mathcal{C}}_{j}(U): X \notin \mathcal{S}_{j}} \bar{\epsilon}^{1+f_{j}\left(a^{(2)}, X\right)} \\
& \leq r^{(2)} C^{|U|_{j}} \bar{\epsilon}^{1+a^{(2)} \eta|U|_{j+1}-2^{d}} \\
& \leq r^{(2)} C^{|U|_{j}} \bar{\epsilon}^{\left(a^{(2)} \eta-a^{(3)}\right)|U|_{j+1}} \bar{\epsilon}^{1+f_{j}\left(a^{(3)}, U\right)},
\end{aligned}
$$

where $\overline{\mathcal{C}}_{j}(U)$ is defined by (5.12). By taking $\bar{\epsilon}$ small, this contribution is negligible compared to the contribution due to $X \in \mathcal{S}_{j}$. 
Finally, we show that the remainder term $R$ of $(5.13)$ is negligible compared with the term that contains $k$.

Lemma 5.7. For $\bar{\epsilon}$ small depending on $L$, and for all $U \in \mathcal{C}_{j+1}$,

$$
\|R(U)\|_{j+1} \prec \bar{\epsilon}^{u+1+f_{j+1}\left(a^{(3)}, U\right)} .
$$

Proof. By (5.13)

$$
R(U)=K^{(3)}(U)-\tilde{I}_{\mathrm{pt}}^{U}[h(U)+k(U)] .
$$

To obtain a convenient expression for (5.41), we introduce $\vec{X}=\left(X_{K}, X_{\delta I}, X_{\tilde{I}_{\mathrm{pt}}}\right)$, and say that $\vec{X} \in \mathcal{X}(U)$ if $X_{K}, X_{\delta I}, X_{\tilde{I}_{\mathrm{pt}}}$ are disjoint sets in $\mathcal{P}_{j}$ whose union is $U$ and also $X_{K} \cup X_{\delta I} \in \overline{\mathcal{P}}_{j}(U)$. We set

$$
\begin{aligned}
n_{K} & =\text { number of components of } X_{K}, \\
n_{\delta I} & =\left|X_{\delta I}\right|_{j}, \\
n & =\left(n_{K}, n_{\delta I}\right) .
\end{aligned}
$$

Then

$$
R(U)=\sum_{\vec{X} \in \mathcal{X}(X)} \mathbb{1}_{n \in N_{0}^{c}} \mathbb{E}_{+}\left(\delta I^{X_{\delta I}} K^{(2)}\left(X_{K}\right)\right)\left(I^{(3)}\right)^{X_{\tilde{I}_{\mathrm{pt}}}},
$$

where $N_{0}^{c}$ is the complement of $N_{0}=\left\{\left(0, \mathbb{N}_{0}\right),(1,0)\right\}$.

With the value 2 arising in (3.19), we write

$$
\alpha=\max \left\{\alpha_{\mathbb{E}}, 2\right\} .
$$

By the product property [20, (1.44)] of the norm, (5.46), (5.17), and Lemma 4.3,

$$
\begin{aligned}
\|R(U)\|_{j+1} & \leq \sum_{\vec{X} \in \mathcal{X}(X)} \mathbb{I}_{n \in N_{0}^{c}} \alpha^{\left|X_{K}\right|_{j}+\left|X_{\delta I}\right|_{j}} \bar{\epsilon}^{\left|X_{\delta I}\right|_{j}}\left\|K^{(2)}\left(X_{K}\right)\right\|_{j} \alpha^{\mid X_{\tilde{I}_{\mathrm{pt}}} j_{j}} \\
& \leq \alpha^{|X|_{j}} \sum_{\vec{X} \in \mathcal{X}(X)} \mathbb{I}_{n \in N_{0}^{c}} \bar{\epsilon}^{\left|X_{\delta I}\right|_{j}} \prod_{i}\left(r^{(2)} \bar{\epsilon}^{1+f_{j}\left(a^{(2)}, X_{K, i}\right)}\right) \\
& \leq(c \alpha)^{L^{d}|X|_{j+1}} \sum_{\vec{X} \in \mathcal{X}(X)} \mathbb{I}_{n \in N_{0}^{c}} \bar{\epsilon}^{\left|X_{\delta I}\right|_{j}} \bar{\epsilon}^{\sum_{i} f_{j}\left(a^{(2)}, X_{K, i}\right)},
\end{aligned}
$$

where the product over $i$ is a product over the connected components $X_{K, i}$ of $X_{K}$. By Lemma C.4 this gives

$$
\|R(U)\|_{j+1} \leq(c \alpha C(L))^{L^{d}|X|_{j+1}} \bar{\epsilon}^{\delta|X|_{j+1}+u+1+f_{j+1}\left(a^{(3)}, X\right)} \sum_{\vec{X} \in \mathcal{X}(X)} \mathbb{I}_{n \in N_{0}^{c}} .
$$

Since the number of terms in the sum over $\vec{X} \in \mathcal{X}(U)$ is at most the number of ways of assigning each $j$-block in $U$ to either $X_{K}, X_{\delta I}$, or $X_{\tilde{I}_{\mathrm{pt}}}$, which is $3^{|U|_{j}}=3^{L^{d}|U|_{j+1}}$, this gives

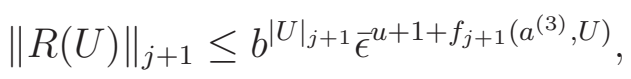

with $b=\bar{\epsilon}(3 c \alpha C(L))^{L^{d}}$. This completes the proof since $b \leq 1$ for $\bar{\epsilon}$ sufficiently small. 


\subsection{Map 4: Reapportionment of $K^{(3)}$}

Map 4 removes the second-order perturbative contribution $h_{\text {lead }}$ from $K^{(3)}$ when $K=0$. Part (iii) is the purpose of this map - the leading part of $K^{(3)}$ has been reapportioned and is now absent in $K^{(4)}$. All norms in this section are scale $j+1$ norms, either the $T_{0, j+1}$ or the $\tilde{G}_{j+1}$ norms, with their corresponding $\mathcal{F}$ norms. We drop the label $j+1$ on the norms, to simplify the notation.

Lemma 5.8. There exist $\mu_{4} \geq 1, K^{(4)}$, and a constant $C=C(L)$ such that, for $(V, K)$ obeying (3.15),

$$
\begin{array}{ll}
\text { (i) } & \tilde{I}_{\mathrm{pt}} \circ K^{(3)}=\tilde{I}_{\mathrm{pt}} \circ K^{(4)}, \\
\text { (ii) } & K^{(4)} \in B_{\mathcal{F}^{(4)}}\left(r^{(4)} \bar{\epsilon}\right), \\
\text { (iii) } & \left\|K^{(4)}(U)\right\| \leq(C \bar{\epsilon})^{f_{j+1}\left(3, a^{(4)}, U\right)} \quad \text { for } U \in \mathcal{P} \quad \text { if } \quad K=0 .
\end{array}
$$

Proof. (i) Let $\mu_{4}=2$, so that $r^{(4)}=2 r^{(3)}$. By Proposition 5.4 and by taking $\tilde{g}$ sufficiently small, there is a constant $C$ such that

$$
\left\|\tilde{I}_{\mathrm{pt}}(U) h_{\text {lead }}(U, B)\right\| \leq(C \bar{\epsilon})^{2} \leq \frac{1}{2} r^{(4)} \bar{\epsilon} .
$$

We apply Proposition 4.1 with $J(U, B)=h_{\text {lead }}(U, B)$ and

$$
\begin{gathered}
I_{\text {in }}=\tilde{I}_{\mathrm{pt}}, \quad K_{\mathrm{in}}=K^{(3)}, \quad \rho=\bar{\epsilon}, \quad a_{\text {in }}=a^{(3)}, \\
a_{\text {out }}=a^{(4)}, \quad \epsilon=\frac{1}{2} r^{(4)}, \quad z=z^{\prime}=1,
\end{gathered}
$$

and we set $K^{(4)}=K_{\text {out }}$. Hypothesis (4.2) is provided by (5.19). Hypothesis (4.3) holds by Lemma F.3, and by the use of $\mathbb{E}_{\pi}$ in (5.18) to localise observables properly. The stability hypothesis (4.4) holds by Proposition 3.1. Hypothesis (4.6) holds by (5.50). Hypothesis (4.7) is obtained from (5.50) and Lemma 5.2(ii) by using the triangle inequality in the definition (4.5) of $M$. Thus all hypotheses hold and Proposition 4.1 implies (i).

(ii) The relevant estimate follows from Proposition 4.1 and (4.11). The fact that $K^{(4)}$ has the properties required of membership in the space $\mathcal{F}^{(3)}$ can be established similarly to what was done in Proposition 5.1 for $K^{(3)}$.

(iii) We again apply Proposition 4.1 but with different choices for parameters. We continue to take $J(U, B)=h_{\text {lead }}(U, B)$, and use the first inequality of (5.50). With Lemma 5.2(iii), (4.5) now gives

$$
M(U)=\tilde{I}_{\mathrm{pt}}^{U}\left(h-h_{\text {lead }}\right)(U) .
$$

By Proposition 5.4,

$$
\|M(U)\|=\left\|\tilde{I}_{\mathrm{pt}}(U)\left(h-h_{\mathrm{lead}}\right)(U)\right\| \leq(C \bar{\epsilon})^{f_{j+1}\left(3, a^{(3)}, U\right)} .
$$

We apply Proposition 4.1 with $\rho=C \bar{\epsilon}, \epsilon=1$, and with $z=1$ replaced by $z=3$. We have the required inequality $z^{\prime}=2>\frac{1}{2} z=\frac{3}{2}$. All hypotheses of Proposition 4.1 then hold with the new parameter values. The conclusion of Proposition 4.1 then implies that

$$
\left\|K^{(4)}(U)\right\| \leq 2(C \bar{\epsilon})^{f_{j+1}\left(3, a^{(4)}, U\right)}
$$

and by absorbing the factor 2 into $C$ we complete the proof of (iii). 
The restriction and isometry properties of $K^{(4)}$ are verified as in maps 1 and 2. In particular, the restriction property is a consequence of the change of variables formula (D.13). For the mass continuity and the $(V, K)$-analyticity, the new ingredient compared to what we have seen in previous Maps is to establish the mass continuity and analyticity of $\tilde{I}_{\mathrm{pt}}^{U} J(U, B)=\tilde{I}_{\mathrm{pt}}^{U} h_{\text {lead }}(U, B)$, with $h_{\text {lead }}(U, B)$ the degree-6 polynomial in the fields defined by (5.18). The subscript $\pi$ in (5.18) is not relevant for the continuity or analyticity properties, and it suffices to verify mass continuity and $(V, K)$-analyticity for the case where $\mathbb{E}_{\pi}$ is replaced by $\mathbb{E}_{+}$in $(\underline{5.18})$. As in the proof of analyticity in Map 1, what is needed is to establish mass continuity and $V$-analyticity (for there is no $K$-dependence) of $h_{\text {lead }}(U, B)$ as a map into $\mathcal{N}\left(U^{\square}\right)$ with the $T_{0}$ norm. The analyticity follows from the fact that $h_{\text {lead }}$ is a bilinear function of $V$, by Lemma 3.5. The mass continuity of $K^{(4)}(U)$ for each $U$ follows from Proposition B.2 and the continuity of $K^{(3)}(U)$ for each $U$.

\section{Maps 5-6: Final adjustments}

The interaction output by Map 4 is $\tilde{I}_{\mathrm{pt}}(b)=e^{-V_{\mathrm{pt}}(b)}\left(1+W_{j+1}\left(V_{\mathrm{pt}}, b\right)\right)$, which involves blocks $b$ of scale $j$. Also, the polynomial $V_{\mathrm{pt}}$ contains monomials $\tau_{\nabla \nabla}$ and $\sigma \bar{\sigma}$ arising in $Q$, and hence does not lie in $\mathcal{Q}^{(0)}$. The purpose of Maps 5-6 is to perform the bookkeeping tasks of replacing $\tilde{I}_{\mathrm{pt}}$ by a scale $j+1$ interaction of the form (1.13), and replacing $V_{\mathrm{pt}}$ by $V_{+}=V_{\mathrm{pt}}^{(0)} \in \mathcal{Q}^{(0)}$. As in (1.73), $V_{\mathrm{pt}}^{(0)}$ is the polynomial obtained by dropping the $\sigma \bar{\sigma}$ term, and by replacing $z \tau_{\Delta}+y \tau_{\nabla \nabla}$ in $V_{\mathrm{pt}}$ by $(z-y) \tau_{\Delta}$ as a formal summation by parts would suggest. Then $V_{+}$and $I_{+}=I_{j+1}\left(V_{+}\right)$have the same form as the initial $V$ and $I=I_{j}(V)$.

To accomplish this we use two steps. First, in Map 5 we eliminate blocks $b \in \mathcal{B}_{j}$ in favour of blocks $B \in \mathcal{B}_{j+1}$, and we simultaneously adjust $W\left(V_{\mathrm{pt}}\right)$ to $W\left(V_{+}\right)$. Second, in Map 6 we replace $e^{-V_{\mathrm{pt}}(B)}$ by $e^{-V_{+}(B)}$ to obtain $I_{+}=I_{j+1}\left(V_{+}\right)$with corresponding $K_{+}$.

Norms in this section are scale $j+1$ norms, either the $T_{0, j+1}$ or the $\tilde{G}_{j+1}$ norms, with their corresponding $\mathcal{F}$ norms. We drop the label $j+1$ on the norms, to simplify the notation.

\subsection{Map 5: Adjustment to $W$}

For $B \in \mathcal{B}_{j+1}$, we define

$$
I_{\mathrm{pt}}^{+}(B)=e^{-V_{\mathrm{pt}}(B)}\left(1+W_{j+1}\left(V_{+}, B\right)\right)
$$

and then define $\delta^{+} I(B)$ by

$$
\tilde{I}_{\mathrm{pt}}(B)=I_{\mathrm{pt}}^{+}(B)+\delta^{+} I(B)
$$

We write

$$
\begin{aligned}
& \delta I_{\mathrm{pt}}^{+}(B)=e^{-V_{\mathrm{pt}}(B)}\left(W_{j+1}\left(V_{\mathrm{pt}}, B\right)-W_{j+1}\left(V_{+}, B\right)\right), \\
& \Delta I(B)=e^{-V_{\mathrm{pt}}(B)}\left(\prod_{b \in \mathcal{B}_{j}(B)}\left(1+W_{j+1}\left(V_{\mathrm{pt}}, b\right)\right)-\left(1+W_{j+1}\left(V_{\mathrm{pt}}, B\right)\right)\right),
\end{aligned}
$$

so that

$$
\delta^{+} I(B)=\Delta I(B)+\delta I_{\mathrm{pt}}^{+}(B)
$$


It is proved in [20, Lemma B.1] (with $j$ replaced by $j+1$ ) that

$$
\|\Delta I(B)\| \prec_{L} \bar{\epsilon}^{4} .
$$

To estimate (6.3), we first recall from (3.24) that $\|Q(b)\|_{T_{0, j}} \prec r^{(0)} \bar{\epsilon}$ for $b \in \mathcal{B}_{j}$. Since the $\tau_{\nabla \nabla}$ term in $V_{\mathrm{pt}}$ arises solely from a contribution due to $Q$, it then follows from [20, Lemma 3.1] that $\left\|y \tau_{\nabla \nabla}(b)\right\|_{T_{0, j}} \prec \bar{\epsilon}$. Thus we can apply [20, Lemma B.3] to conclude that

$$
\left\|\delta I_{\mathrm{pt}}^{+}(B)\right\| \prec_{L} \bar{\epsilon}^{2}
$$

(note that in [20, Lemma B.3] the $\sigma \bar{\sigma}$ term is not eliminated when creating $V_{+}$, but such a term in $V$ does not contribute to $W(V)$ by definition, so [20, Lemma B.3] does apply).

Lemma 6.1. There exist $\mu_{5} \geq 1, K^{(5)}$, and a constant $C=C(L)$ such that, for $(V, K)$ obeying (3.15),

$$
\begin{aligned}
\text { (i) } & \left(\tilde{I}_{\mathrm{pt}} \circ K^{(4)}\right)(\Lambda)=\left(I_{\mathrm{pt}}^{+} \circ K^{(5)}\right)(\Lambda), \\
\text { (ii) } & K^{(5)} \in B_{\mathcal{F}^{(5)}}\left(r^{(5)} \bar{\epsilon}\right), \\
\text { (iii) } & \left\|K^{(5)}(U)\right\| \leq(C \bar{\epsilon})^{f_{j+1}\left(z_{h}, a^{(5)}, U\right)} \quad \text { for } U \in \mathcal{P} \quad \text { if } \quad K=0 .
\end{aligned}
$$

Proof. (i) Let

$$
K^{(5)}=K^{(4)} \circ \delta^{+} I
$$

By (6.2) and Lemma 1.3,

$$
K^{(4)} \circ \tilde{I}_{\mathrm{pt}}=K^{(4)} \circ\left(\delta^{+} I \circ I_{\mathrm{pt}}\right)=K^{(5)} \circ I_{\mathrm{pt}}^{+} .
$$

(ii) By (6.5)-(6.7), for $\bar{\epsilon}$ sufficiently small we have

$$
\left\|\delta I^{+}(B)\right\| \leq C \bar{\epsilon}^{2} \leq r^{(4)} \bar{\epsilon}
$$

i.e., $\delta I^{+} \in B_{\mathcal{F}^{(4)}}\left(r^{(4)} \bar{\epsilon}\right)$. The desired estimate is then a consequence of Lemmas 5.8 and C.1, once we set $r^{(5)}=\mu_{5} r^{(4)}$ with $\mu_{5}=2^{2^{d}}$. The vanishing at weighted infinity, field locality, symmetry, and component factorisation properties inherent in the statement that $K^{(5)} \in \mathcal{F}^{(5)}$ can be verified using the fact that $K^{(4)}$ has these properties. (In particular, $\delta^{+} I$ vanishes at weighted infinity since each of $\tilde{I}_{\mathrm{pt}}$ and $I_{\mathrm{pt}}^{+}$do by Proposition 3.3.)

(iii) This follows from the first inequality of (6.10) and from Lemma 5.8(iii), by Lemma C.1.

The restriction and isometry properties of $K^{(5)}$ can be verified as in maps 1 and 2 using its definition, as can the mass continuity $m^{2} \mapsto K^{(5)}(U) \in \mathcal{N}\left(U^{\square}\right),\|\cdot\|_{j+1}$ and the $(V, K)$-analyticity, using the corresponding properties of $K^{(4)}$. For the analyticity, slight modifications to the proof of [20, Proposition 2.3] show that the map $(V, K) \mapsto I_{\mathrm{pt}}^{+}$is analytic from $\mathcal{D}_{j} \times B_{\mathcal{F}_{j}}\left(r^{(0)} \bar{\epsilon}\right)$ into $\mathcal{N}\left(B^{\square}\right),\|\cdot\|_{j+1}\left(\right.$ and so is $\left.(V, K) \mapsto I_{+}\right)$. 


\subsection{Map 6: Adjustment to $V$}

As in (1.73), we define

$$
I^{(6)}=I_{+}=e^{-V_{+}}\left(1+W_{j+1}\left(V_{+}\right)\right)
$$

Map 6 performs two tasks. First, it removes the monomial $y \tau_{\nabla \nabla}$ from the exponent of $I_{\mathrm{pt}}^{+}$by converting it to $y \tau_{\Delta}$ by summation by parts. Second, it extracts $\delta q \sigma \bar{\sigma}$ from $I_{\mathrm{pt}}^{+}$to bring it out of the circle product; here $\delta q=\frac{1}{2}\left(\delta q^{a}+\delta q^{b}\right)$ as in (1.78). The boundary term resulting from the first task, and an adjustment to achieve the second, convert $K^{(5)}$ to $K_{+}=K^{(6)}$. All objects in this section are at scale $j+1$.

In more detail, for $Z \in \mathcal{P}_{j+1}$ we define

$$
V_{\partial, Z}=\sum_{z \in Z} y_{\mathrm{pt}}\left(\tau_{\nabla \nabla, z}-\tau_{\Delta, z}\right)
$$

With the definition of $I_{\mathrm{pt}}^{+}$in (6.1), this gives

$$
I_{\mathrm{pt}}^{+}(Z)=e^{v(Z)} I_{+}(Z) e^{-V_{\partial, Z}},
$$

where $v(Z)=\sigma \bar{\sigma} \frac{1}{2}\left(\delta q^{a} \mathbb{1}_{a \in Z}+\delta q^{b} \mathbb{1}_{b \in Z}\right)$ (cf. $V_{a b}$ in (1.8)). By performing summation by parts on the right-hand side of (6.12), we find that there exists $V_{\partial, Z, B}$, which depends only on fields that are in the intersection of $B$ and the boundary of $Z$, such that

$$
V_{\partial, Z}=\sum_{B \in \mathcal{B}} V_{\partial, Z, B}
$$

Here $V_{\partial, Z, B}=0$ if $B$ is not a block in $Z$ which is on the boundary of $Z$ in the sense that it has a neighbour not in $Z$ (in particular, $V_{\partial, \Lambda, B}=0$ ).

We therefore have

$$
I_{\mathrm{pt}}^{+}(\Lambda \backslash X)=e^{v(\Lambda \backslash X)} \prod_{B \in \mathcal{B}_{j+1}(\Lambda \backslash X)} I_{+}(B)\left(1+R_{X}(B)\right) \quad \text { with } \quad R_{X}(B)=e^{-V_{\partial, \Lambda \backslash X, B}}-1 .
$$

By Lemma 1.3,

$$
I_{\mathrm{pt}}^{+}(\Lambda \backslash X)=e^{v(\Lambda \backslash X)}\left(\delta I_{X}^{(6)} \circ I_{+}\right)(\Lambda \backslash X)=e^{v(\Lambda \backslash X)} \sum_{Y \in \mathcal{P}_{j+1}(\Lambda \backslash X)}\left(\delta I_{X}^{(6)}\right)^{Y} I_{+}^{\Lambda \backslash(X \cup Y)},
$$

where

$$
\left(\delta I_{X}^{(6)}\right)^{Y}=\prod_{B \in \mathcal{P}_{j+1}(Y)} R_{X}(B) I_{+}(B) .
$$

Note that $\delta I_{\varnothing}^{(6)}=0$ by definition. It follows from [20, Lemma B.3] (together with the verification of its assumption as above (6.7)) that there is a constant $c$ such that

$$
\left\|\delta I_{X}^{(6)}(B)\right\|_{j+1} \leq c \bar{\epsilon} .
$$


Lemma 6.2. There exist $\mu_{6} \geq 1$ and $K^{(6)}=K_{+}$such that, for $(V, K)$ obeying (3.15),

$$
\begin{array}{ll}
\text { (i) } & I_{\mathrm{pt}}^{+} \circ K^{(5)}=I_{+} \circ K_{+}, \\
\text {(ii) } & K_{+} \in B_{\mathcal{F}^{(6)}}\left(r^{(6)} \bar{\epsilon}\right), \\
\text { (iii) } & \left\|K_{+}(U)\right\| \leq(C \bar{\epsilon})^{f_{j+1}\left(z_{h}, a^{(6)}, U\right)} \quad \text { for } U \in \mathcal{P} \quad \text { if } K=0 .
\end{array}
$$

Proof. (i) It follows from (6.16), and from the formula for $\delta q$ in (1.78), that

$$
\begin{aligned}
\left(I_{\mathrm{pt}}^{+} \circ K^{(5)}\right)(\Lambda) & =e^{\delta q \sigma \bar{\sigma}} \sum_{X \in \mathcal{P}(\Lambda)} e^{-v(X)} K^{(5)}(X)\left(\delta I_{X}^{(6)} \circ I_{+}\right)(\Lambda \backslash X) \\
& =e^{\delta q \sigma \bar{\sigma}} \sum_{Z \in \mathcal{P}(\Lambda)} \sum_{X \in \mathcal{P}(Z)} e^{-v(X)} K^{(5)}(X)\left(\delta I_{X}^{(6)}\right)^{Z \backslash X} I_{+}^{\Lambda \backslash Z} \\
& =e^{\delta q \sigma \bar{\sigma}}\left(I_{+} \circ K_{+}\right)(\Lambda)
\end{aligned}
$$

where, for $Z \in \mathcal{P}$, we define

$$
K_{+}(Z)=\sum_{X \in \mathcal{P}(Z)} e^{-v(X)} K^{(5)}(X)\left(\delta I_{X}^{(6)}\right)^{Z \backslash X} .
$$

(ii) It follows from the product property of the $T_{\phi}$ norm that $\left\|e^{-v(X)}\right\| \leq e^{\|v(X)\|}$ (for a proof, see [18, (5.26)]). By definition, $\|v(X)\| \leq|\delta q| \mathfrak{h}_{\sigma}^{2}$. Moreover, $\delta q$ is non-zero only when $j$ is at least the coalescence scale $j_{a b}$, and in this case $Q$ no longer contains a $\lambda$ term since the corresponding monomials are no longer in the range of Loc above coalescence (see [7, Section 3.2]). Therefore, by [7, Proposition 4.1] and [7, (3.35)], $|\delta q| \prec \lambda^{2} L^{-2 j} \prec L^{-2 j}$. This implies that $\|v(X)\| \prec L^{-2 j} \mathfrak{h}_{\sigma}^{2}$, and from the definitions of $\mathfrak{h}_{\sigma}$ in (1.32), this shows that $\left\|e^{-v(X)}\right\| \leq 2$. With Lemmas 6.1 and C.2, and by (6.18), this gives

$$
\begin{aligned}
\left\|K_{+}(Z)\right\| & \leq \sum_{X \in \mathcal{P}(Z): X \neq \varnothing}\left\|e^{-v(X)}\right\|\left\|K^{(5)}(X)\right\|\left\|\delta I_{X}^{(6)}\right\|^{|Z \backslash X|} \\
& \leq 2 r^{(5)} \sum_{X \in \mathcal{P}(Z): X \neq \varnothing} \bar{\epsilon}^{1+f\left(a^{(5)}, X\right)}(c \bar{\epsilon})^{|Z \backslash X|} \\
& \leq 2 r^{(5)}(2 c)^{|Z|} \sup _{X \in \mathcal{P}(Z): X \neq \varnothing} \bar{\epsilon}^{1+f\left(a^{(5)}, X\right)+|Z \backslash X|} \\
& \leq 2 r^{(5)}(2 c)^{2^{d}} \bar{\epsilon}^{1+f\left(a^{(6)}, Z\right)}=r^{(6)} \bar{\epsilon}^{1+f\left(a^{(6)}, Z\right)}
\end{aligned}
$$

where in the last step we set $r^{(6)}=\mu_{6} r^{(5)}$ with $\mu_{6}=2(2 c)^{2^{d}}$, and used $a^{(6)}$ to cancel the exponential growth of $(2 c)^{|Z|}$ for large sets $Z$. Specialising to the case where $Z$ is connected, we obtain the estimate of (ii).

To see that $K_{+}$obeys the component factorisation property, let $Z$ be the disjoint union of $Z_{1}$ and $Z_{2}$. The sum over $X$ in (6.21) can then be written as the sum over $X_{1} \in \mathcal{P}\left(Z_{1}\right)$ and $X_{2} \in \mathcal{P}\left(Z_{2}\right)$, and the component factorisation property of $K^{(5)}$ implies that $K^{(5)}(X)=K^{(5)}\left(X_{1}\right) K^{(5)}\left(X_{2}\right)$. It suffices if $\left(\delta I_{X_{1} \cup X_{2}}^{(6)}\right)^{Z \backslash\left(X_{1} \cup X_{2}\right)}=\left(\delta I_{X_{1}}^{(6)}\right)^{Z_{1} \backslash X_{1}}\left(\delta I_{X_{2}}^{(6)}\right)^{Z_{2} \backslash X_{2}}$, and this indeed holds because

$$
\prod_{B \in \mathcal{B}\left(Z \backslash\left(X_{1} \cup X_{2}\right)\right.} R_{X_{1} \cup X_{2}}(B)=\prod_{B \in \mathcal{B}\left(Z_{1} \backslash X_{1}\right)} R_{X_{1}}(B) \prod_{B \in \mathcal{B}\left(Z_{2} \backslash X_{2}\right)} R_{X_{2}}(B) .
$$


The fact that $K^{(6)}$ obeys the field locality, symmetry and component factorisation properties can be seen from its definition. The fact that $K^{(6)}$ vanishes at weighted infinity follows from the fact that $K^{(5)}$ does, together with the fact that $\delta I_{X}^{(6)}$ vanishes at weighted infinity by an extension of Proposition 3.3 ,

(iii) When $K=0$, by Lemma 6.1(iii) we can replace $r^{(5)}$ in the proof of part (ii) by $C \bar{\epsilon}^{z_{h}}$, and this immediately gives the result.

The restriction and isometry properties are straightforward to verify as in maps 1 and 2 . We omit the tedious details which justify the mass continuity $m^{2} \mapsto K^{(6)}(U) \in \mathcal{N}\left(U^{\square}\right),\|\cdot\|_{j+1}$, and the $(V, K)$-analyticity of $K^{(6)}$.

Remark 6.3. Recall the discussion of the 4-dimensional $n$-component $|\varphi|^{4}$ model in Section 1.8.4. We now sketch how Lemma 6.2 can be adapted so that the results of the present paper can be applied in [9] to the $|\varphi|^{4}$ model. The new ingredient is that $V_{\mathrm{pt}}(V-Q)$ contains a constant term $u$, even when $V$ does not, because $Q$ will contain such a term and also $V_{\text {pt }}$ will produce one. Let $|X|_{j}$ denote the number of scale- $j$ blocks in $X \in \mathcal{P}_{j}$. In particular, $|X|_{0}$ is the number of points in $X$. The term $u$ in $V$ occurs in $I(X, V)$ only as an overall factor $e^{\delta u|X|_{0}}$, since a constant term in $V$ cannot contribute to $W$. In the scale- $(j+1)$ circle product considered in Lemma 6.2, we wish to replace the factor $e^{\delta u|X|_{0}}$ multiplying $I_{\mathrm{pt}}^{+}(X)$ by $e^{\delta u|\Lambda|_{0}}$. For this, we use

$$
\left(\left(e^{\delta u} I_{\mathrm{pt}}^{+}\right) \circ K^{(5)}\right)(\Lambda)=e^{\delta u|\Lambda|_{0}}\left(I_{\mathrm{pt}}^{+} \circ\left(e^{-\delta u} K^{(5)}\right)(\Lambda) .\right.
$$

The multiplication of $K^{(5)}$ on the right-hand side is controlled by the estimate

$$
\left\|e^{-\delta u|X|_{0}} K^{(5)}(X)\right\| \leq e^{|\delta u||X|_{0}}\left\|K^{(5)}(X)\right\|=e^{|\delta u| L^{d(j+1)}|X|_{j+1}}\left\|K^{(5)}(X)\right\| .
$$

By definition,

$$
\delta u=\left.V_{\mathrm{pt}}(V-Q)\right|_{\varphi=0}=\left.V_{\mathrm{pt}}(V)\right|_{\varphi=0}+\left(\left.V_{\mathrm{pt}}(V-Q)\right|_{\varphi=0}-\left.V_{\mathrm{pt}}(V)\right|_{\varphi=0}\right) .
$$

As in the proof of Theorem 1.10, we find that

$$
\left|V_{\mathrm{pt}}(V-Q)\right|_{\varphi=0}-\left.V_{\mathrm{pt}}(V)\right|_{\varphi=0} \mid \leq O\left(L^{-4 d} \chi \tilde{g}^{3}\right) .
$$

Since $\left.V_{\mathrm{pt}}(V)\right|_{\varphi=0}=O\left(L^{-d j} \chi \tilde{g}\right)$ by [9, Lemma A.1], this gives

$$
|\delta u|=O\left(L^{-d j} \chi_{j} \tilde{g}_{j}\right)
$$

Therefore,

$$
\left\|e^{-\delta u|X|_{0}} K^{(5)}(X)\right\| \leq e^{O\left(\chi_{j} \bar{g}_{j}\right)|X|_{j+1}}\left\|K^{(5)}(X)\right\| .
$$

The small amount of exponential growth on the right-hand side is handled by the increase from $a^{(5)}$ to $a^{(6)}$ which already performs a similar task in the proof of Lemma 6.2. Other aspects of the proof of our main results are unchanged, and we apply this extension in [9]. 


\section{Completion of proof of Theorem 2.2}

We now assemble the conclusions obtained in the analysis of the six Maps, to complete the proof of Theorem 2.2.

Proof of Theorem 2.2(i). Since $K_{+}$is the composition of the six maps, the domain of $K_{+}$is the domain of the first map $K^{(1)}$, which, as specified in the hypothesis of Lemma 4.2. is $\mathcal{D} \times B_{\mathcal{F}}(r \bar{\epsilon})$ as desired. The range of $K_{+}$is the range of the sixth map $K^{(6)}$. By Lemma $6.2\left(\right.$ ii), $K^{(6)} \in$ $B_{\mathcal{F}_{j+1}\left(a^{(6)}\right)}\left(r^{(6)} \bar{\epsilon}\right)$. From Section 3.2. we find that $r^{(6)}=\kappa r$, so $K^{(6)} \in B_{\mathcal{F}_{j+1}\left(a^{(6)}\right)}(\kappa r \bar{\epsilon})$. By (1.30) and (1.43),, $\bar{\epsilon} / \bar{\epsilon}_{j+1}$ is bounded by a constant, so we can replace $\bar{\epsilon}$ by $\bar{\epsilon}_{+}$by absorbing this constant into the constant $\gamma^{*}$ in $\kappa=O\left(L^{-1}\right)$. By $(3.14), a^{(6)}>a^{(0)}=a$, so the output space $\mathcal{F}_{j+1}\left(a^{(6)}\right)$ has $a_{+}=a^{(6)}>a$ as claimed. Furthermore, by [20, Remark 1.3], all estimates involving our norm pairs in [20] remain valid for some choice of $\mathfrak{h}_{++}>\mathfrak{h}_{+}$. For this reason, $\mathfrak{h}_{+}$can be replaced by $\mathfrak{h}_{++}$ as required.

The bound (2.10) is obtained in Lemma 6.2(iii), again with a larger constant to allow the replacement of $\bar{\epsilon}$ by $\bar{\epsilon}_{+}$as explained above. The fact that the circle product is preserved in the sense of (1.49) is a consequence of part (i) of Lemmas 4.3, 5.2, 5.8, 6.1 and 6.2, The desired $(V, K)$ analyticity is a consequence of the analyticity established for each Map.

Proof of Theorem 2.2(ii-iii). These have both been established for the six individual Maps and therefore hold for $K_{+}$.

Proof of Theorem 2.2(iv). For mass continuity, the mass $m^{2}$ which is being varied appears in the analysis via the mass dependence of the covariance $C_{+}$, which is a member of the decomposition of the covariance $\left(-\Delta+m^{2}\right)^{-1}$. The mass continuity established for the six Maps provides a statement of continuity of $K_{+}$as a map from $m^{2} \in \mathbb{I}_{+}$into the space $\mathcal{N}\left(U^{\square}\right)$, $\|\cdot\|_{j+1}$, for each polymer $U \in \mathcal{P}_{+}$.

We wish to transfer this into a statement of continuity of $K_{+}$as a map from $m^{2} \in \tilde{\mathbb{I}}_{+}\left(\tilde{m}^{2}\right)$ into $\mathcal{F}_{+}$. The value of $\tilde{m}^{2}$ fixes the space $\tilde{\mathcal{F}}_{+}$and fixes $\tilde{\chi}$ which determines the radius of balls in this space, so that neither the space nor the ball varies with $m^{2}$. By [7, (4.22)], $\chi_{j}=\Omega^{-\left(j-j_{\Omega}\right)_{+}} \asymp$ $\Omega^{-\left(j-j_{m}\right)_{+}}$, where $j_{m}=\left\lfloor\log _{L^{2}} m^{-2}\right\rfloor$. The values of $m^{2}$ and $\tilde{m}^{2}$ are comparable by definition of $\tilde{\mathbb{I}}$, hence so are $j_{m}$ and $j_{\tilde{m}}$, and hence so are $\chi\left(m^{2}\right)$ and $\tilde{\chi}=\chi\left(\tilde{m}^{2}\right)$. Consequently, $\bar{\epsilon}$ of (1.43) differs by a constant factor when computed using $\chi$ or $\tilde{\chi}$. The estimates of Propositions 5.3 5.4 produce $\bar{\epsilon}$ constructed from $\mathrm{m}^{2}$, since these are estimates based on the covariance $C_{+}$. On the other hand, estimates implied by membership in the space $\tilde{\mathcal{F}}_{+}$are in terms of $\bar{\epsilon}$ constructed from $\tilde{m}^{2}$, by definition. The fact that the two versions of $\bar{\epsilon}$ are comparable means that it does not matter if different versions appear at different steps of the analysis. As there are only finitely many polymers $U$ in $\Lambda$, the continuity of $K_{+}$as a map into $\mathcal{N}(U),\|\cdot\|_{j+1}$ therefore implies continuity into $\mathcal{F}_{+}$, as required.

Proof of Theorem 2.2( $(v)$. We consider the case $x=a$, as the case $x=b$ is similar. If $\pi_{a} V=0$ and $\pi_{a} K(X)=0$ for all $X \in \mathcal{P}$, then neither $I$ nor $K$ has a component in $\pi_{a} \mathcal{N}$, and the observable field $\sigma$ is not present in either of $I$ or $K$. It is possible that $\bar{\sigma}$ or $\sigma \bar{\sigma}$ are present in $K$. However, in our construction of $K_{+}$via the six Maps, the operations involving the observable fields consist of 
multiplication of polynomials in the quotient space discussed around (1.2). Therefore no $\sigma$ term, i.e. no term in $\pi_{a} \mathcal{N}$, can be created in $K_{+}$if it is not present initially.

\section{A Proof of Proposition 1.8}

In this section we prove Proposition 1.8, which states that several normed spaces are complete.

We fix the scale $j$ and suppress it in the notation. Thus $\mathcal{C}(\mathbb{V})$ is the set of connected polymers at scale $j$. For $X \in \mathcal{C}(\mathbb{V})$, let $W(X, \phi)$ be a continuous positive function of $\phi$ in the normed space $\Phi\left(X^{\square}\right)$. This means that $W(X, \phi)$ is a function of $\phi$ in the space of fields in $\phi: \mathbb{V} \rightarrow \mathbb{C}$ but only depends on the restriction of $\phi$ to $X^{\square}$. Let $\mathcal{S}(\mathbb{V})$ be the space of maps $F: \mathcal{C}(\mathbb{V}) \rightarrow \mathcal{N}(\mathbb{V})$ such that $F(X)$ is in $\mathcal{N}\left(X^{\square}\right)$ for $X$ in $\mathcal{C}(\mathbb{V})$. The following proposition provides the first step in the proof of Proposition 1.8 .

Proposition A.1. For $\mathbb{V}=\Lambda$ or $\mathbb{V}=\mathbb{Z}^{d}$ the space $\mathcal{S}(\mathbb{V})$ is complete in the norm

$$
\|F\|_{W}=\sup _{X \in \mathcal{C}(\mathbb{V}), \phi \in \Phi(\mathbb{V})}\|F\|_{T_{\phi}} W^{-1}(X, \phi) .
$$

Proof. We suppress the $\mathbb{V}$ argument. For $X \in \mathcal{C}, \phi \in \Phi, g \in \Phi$, define the linear functional

$$
\lambda_{X, \phi, g}: \mathcal{S} \rightarrow \mathbb{C} \quad \text { by } \quad F \mapsto\langle F(X), g\rangle_{\phi} W^{-1}(X, \phi),
$$

with the pairing on the right-hand side defined in [18, Definition 3.3]. Then

$$
\|F\|_{\mathcal{S}}=\sup _{X \in \mathcal{C}, \phi \in \Phi, g \in B(\Phi)}\left|\lambda_{X, \phi, g}(F)\right| .
$$

Therefore a sequence $F_{n}$ in $\mathcal{S}$ is Cauchy if and only if $\lambda_{X, \phi, g}\left(F_{n}\right)$ is Cauchy in $\mathbb{C}$, uniformly in the parameters $(X, \phi, g) \in \mathcal{C} \times \Phi \times B(\Phi)$. Let $F_{n}$ be a Cauchy sequence in $\mathcal{S}$. By completeness of $\mathbb{C}$ the sequence $\lambda_{X, \phi, g}\left(F_{n}\right)$ has a limit $F_{X, \phi, g}$ in $\mathbb{C}$. Since $F_{n}$ is uniformly Cauchy the convergence is uniform in the parameters. Therefore, to prove that $\mathcal{S}$ is complete, it suffices to prove that there exists $F$ in $\mathcal{S}$ such that $\lambda_{X, \phi, g}(F)=F_{X, \phi, g}$ for all values of the parameters. Thus we fix $X \in \mathcal{C}$, assume that $F_{n}$ is a sequence in $\mathcal{N}\left(X^{\square}\right)$, and it suffices to prove that there exists $F \in \mathcal{N}\left(X^{\square}\right)$ such that $\lambda_{X, g, \phi}\left(F_{n}\right) \rightarrow \lambda_{X, g, \phi}(F)$.

It suffices to restrict the test function $g$ to a small class of test functions, as follows. Let $z$ be a sequence in $\Lambda^{*}$ and let $x$ and $y$ be the boson and fermion subsequences of $z$. Let the length $p(x)$ of the sequence $x$ be at most $p_{\mathcal{N}}+2$, where the 2 allows for observables. We define a test function $\delta_{z} \in \Phi$ by setting $\delta_{z}\left(z^{\prime}\right)=1$ when $z^{\prime}=z$ and $\delta_{z}\left(z^{\prime}\right)=0$ otherwise, for $z^{\prime} \in \Lambda^{*}$. By the definition of $\Phi\left(X^{\square}\right)$ all elements of $\Phi\left(X^{\square}\right)$ are finite linear combinations of these special test functions. Thus it suffices to prove that $\lambda_{X, \phi, \delta_{z}}\left(F_{n}\right) \rightarrow \lambda_{X, \phi, \delta_{z}}(F)$ since this gives the corresponding results for all $g$ in $\Phi\left(X^{\square}\right)$ and therefore also for all $g$ in $\Phi$.

Since $\delta_{z}$ can be normalised to be in $B(\Phi), \lambda_{X, \phi, \delta_{z}}\left(F_{n}\right)$ is Cauchy in $\mathbb{C}$, uniformly in $\phi \in \Phi$. By the definition of the pairing,

$$
\left\langle F_{n}, \delta_{z}\right\rangle_{\phi}=F_{n, x, y}=\left(\prod_{i=1}^{p(x)} \frac{\partial}{\partial \phi_{x_{i}}}\right) F_{n, y}(\phi)
$$


is a partial derivative of $F_{n, y}$ with respect to $\phi$. By the definition of $\mathcal{S}$ this partial derivative is continuous in $\phi$ and the pairing is well defined on the equivalence classes $\phi \in \Phi\left(X^{\square}\right)$ and $g \in \Phi\left(X^{\square}\right)$. By hypothesis, $W(X, \phi)$ is bounded below uniformly on compact subsets of $\Phi\left(X^{\square}\right)$. Therefore the uniform convergence of $\lambda_{X, \phi, \delta_{z}}\left(F_{n}\right)$ implies that the partial derivative $F_{n, x, y}$ converges uniformly in $\phi$ for $\phi$ in compact subsets of $\Phi\left(X^{\square}\right)$. By the continuity of $F_{n, x, y}$ as a function of $\phi$ the limit of $F_{n, x, y}$ is continuous in $\phi$. By integration we find that the derivatives of the limit are the limits of the derivatives. Therefore there exists $F_{y} \in \mathcal{N}\left(X^{\square}\right)$ such that $F_{n, x, y}(\phi)$ converges to $F_{x, y}(\phi)$ for all $\phi$. Letting $F=\sum_{y} \frac{1}{y !} F_{y}(\phi) \psi^{y}$ and noting that this sum over $y$ is a finite sum because $X$ is a finite set, we have $\lambda_{X, \phi, \delta_{z}}\left(F_{n}\right) \rightarrow \lambda_{X, \phi, \delta_{z}}(F)$, and the proof is complete.

As in Section 1.7, we denote by $\mathcal{I}(\mathbb{V})$ the set of elements of $\mathcal{N}$ whose $T_{0}$ semi-norm is zero. Define $\mathcal{S}\left(T_{0}\right)$ to be the space of maps $F: \mathcal{C}(\mathbb{V}) \rightarrow \mathcal{N}(\mathbb{V}) / \mathcal{I}(\mathbb{V})$ such that $F(X) \in \mathcal{N}\left(X^{\square}\right) / \mathcal{I}(\mathbb{V})$. Since we have factored out the ideal $\mathcal{I}(\mathbb{V})$, the $T_{0}$ semi-norm becomes a norm on this space.

Proposition A.2. For $\mathbb{V}=\Lambda$ or $\mathbb{V}=\mathbb{Z}^{d}$, the space $\mathcal{S}\left(T_{0}\right)$ is complete.

Proof. Given $F \in \mathcal{N}$, we replace $\phi$ and $\psi$ by $t \phi$ and $t \psi$ and construct a polynomial $T \in \mathcal{N}$ of degree $p_{\mathcal{N}}$ by making a Taylor expansion in powers of $t$ to order $p_{\mathcal{N}}$ and setting $t=1$. Then derivatives of $T$ at $\phi=0$ match derivatives of $F$ up to and including order $p_{\mathcal{N}}$. Therefore $F-T \in \mathcal{I}(\mathbb{V})$, and the map $F \mapsto T$ identifies $\mathcal{N}(\mathbb{V}) / \mathcal{I}(\mathbb{V})$ with polynomials of degree $p_{\mathcal{N}}$. Then, for all $X, \mathcal{N}(X) / \mathcal{I}(\mathbb{V})$ is a finite dimensional space and therefore $\mathcal{S}\left(T_{0}\right)$ is complete in $T_{0}$ norm.

Proposition A.3. For either of the two choices $\mathbb{V}=\mathbb{Z}^{d}$ or $\mathbb{V}=\Lambda$, the spaces $\mathcal{F}(G), \mathcal{F}(\tilde{G})$, are closed subspaces of $\mathcal{S}$ and are complete. Likewise, $\mathcal{F}\left(T_{0}\right)$ is a closed subspace of $\mathcal{S}\left(T_{0}\right)$ and is also complete.

Proof. The spaces $\mathcal{F}(G)$ and $\mathcal{F}(\tilde{G})$ are obtained when $W(X, \phi)$ is chosen as in (1.40). According to the definitions of the regulators in [20, (1.38), (1.41)], $W(X, \phi)$ is positive and continuous in $\phi$. Therefore, by Proposition A.1, with either choice of $W$, the space $\mathcal{S}$ is complete. Also, according to Proposition A.2, $\mathcal{S}\left(T_{0}\right)$ is complete. Therefore it is sufficient to prove that $\mathcal{F}(G), \mathcal{F}(\tilde{G})$ and $\mathcal{F}\left(T_{0}\right)$ are closed subspaces. As discussed in Section 1.7.3, elements of $\mathcal{F}(G), \mathcal{F}(\tilde{G})$ and $\mathcal{F}\left(T_{0}\right)$ must satisfy the symmetry and field locality conditions of Definition 1.7. These conditions define closed subspaces.

Therefore, it only remains to prove for the cases $\mathcal{F}(G), \mathcal{F}(\tilde{G})$ that the condition of vanishing at weighted infinity defines a closed subspace of $\mathcal{S}$. For this let $F_{1}, F_{2}, \ldots$ be a sequence of elements of $\mathcal{S}$ that vanish at weighted infinity and are such that the sequence converges in $\mathcal{S}$ to a limit $F$. We must prove $F$ vanishes at weighted infinity. Let $\epsilon>0$ and let $X$ be a polymer. By definition, there exists $N$ such that $\left\|F(X)-F_{N}(X)\right\|_{T_{\phi}} W^{-1}(X, \phi)<\epsilon$ uniformly in $\phi$. Therefore

$$
\|F(X)\|_{T_{\phi}} W^{-1}(X, \phi)<\epsilon+\left\|F_{N}\right\|_{T_{\phi}} W^{-1}(X, \phi) .
$$

Since $F_{N} \in \mathcal{S}$, it follows that

$$
\limsup _{\|\phi\|_{\Phi(X)} \rightarrow \infty}\|F(X)\|_{T_{\phi}} W^{-1}(X, \phi)<\epsilon
$$

and since this holds for all $\epsilon, F$ vanishes at weighted infinity, as was to be proved. 
Proof of Proposition 1.8. A Cauchy sequence $F_{n}$ in $\mathcal{W}(\mathbb{V})$ is Cauchy in each of the $\mathcal{F}(G)$ and $\mathcal{F}(\tilde{G})$ norms. Therefore it has limits $F_{G}$ and $F_{\tilde{G}}$ in the $\mathcal{F}(G)$ norm and the $\mathcal{F}(\tilde{G})$ norm. Both norms imply convergence pointwise in $X, \phi$ so $F_{G}=F_{\tilde{G}}$ and therefore $F_{n}$ is convergent in $\mathcal{W}(\mathbb{V})$.

\section{B Two properties of the expectation}

In this section, we prove that the expectation is continuous in the mass, and that the expectation preserves the property of vanishing at infinity. We begin with the continuity statement.

\section{B.1 Mass continuity of the expectation}

\section{B.1.1 Statement of continuity}

We consider the continuity properties of the expectation as a function of the covariance, and of the mass which defines the covariance. There are two fixed scales, $j$ and $j+1$, and the scale advances in norms when the expectation is taken. We omit the scale when it is $j$ and write + to indicate scale $j+1$. The covariance $C$ is always considered to be a test function with two arguments and, furthermore, is assumed to be in the unit ball $B_{1}\left(\Phi_{+}\right)$of the space $\Phi_{+}$of test functions. Let $X \in \mathcal{C}$ be a connected polymer $X \in \mathcal{C}$. Recall from (1.35)-(1.36) that two norm pairs $\|\cdot\|_{j},\|\cdot\|_{j+1}$ are defined on $\mathcal{N}\left(X^{\square}\right)$. We write $\mathcal{X}$ for either of the normed spaces defined by the two choices of $\|\cdot\|_{j}$ and for each of these choices let $\mathcal{X}_{+}$be the normed spaces defined by the accompanying choice of $\|\cdot\|_{j+1}$. The main continuity result is the following proposition, whose proof is given in the remainder of Section B.1.

Proposition B.1. For $F \in \mathcal{X}$, the map $C \mapsto \mathbb{E}_{C} \theta F$ from $B_{1}\left(\Phi_{+}\right)$to $\mathcal{X}_{+}$is continuous.

Now we choose the covariance $C$ to be one of the $m^{2}$-dependent covariances $C=C_{j+1}$ for $j<N(\mathbb{V})$, or $C=C_{N, N}$ for $j+1=N(\Lambda)$, which arise in the finite-range decomposition of the covariance $\left(-\Delta+m^{2}\right)^{-1}$ described in [20, Section 1.1.1]. Proposition B.1] then implies the continuity of the expectation as a function of the mass $m^{2}$.

Proposition B.2. For $F \in \mathcal{X}$, the map $m^{2} \mapsto \mathbb{E}_{C} \theta F$ from $\mathcal{X}$ to $\mathcal{X}_{+}$is continuous.

Proof. By Proposition B.1, it suffices to show that $m^{2} \mapsto C$ is a continuous function from $I_{j}$ to $B_{1}\left(\Phi_{+}\right)$. This is a consequence of [7, Proposition 6.1(b)]. (In fact, [7, Proposition 6.1] does not directly address mass continuity in the $\Phi_{j+1}$ norm, but it does when augmented with the estimate [4, (1.15)].)

\section{B.1.2 Reduction to dense subset}

The following lemma is a standard result in functional analysis. We omit the proof, which is an $\epsilon / 3$ argument.

Lemma B.3. Let $\mathcal{B}$ and $\mathcal{B}_{+}$be Banach spaces. Suppose that the sequence of linear operators $T_{n}: \mathcal{B} \rightarrow \mathcal{B}_{+}$is uniformly bounded in operator norm, and suppose that $T: \mathcal{B} \rightarrow \mathcal{B}_{+}$is a bounded linear operator. If $T_{n} F \rightarrow T F$ for all $F$ in a dense subset of $\mathcal{B}$, then $T_{n} F \rightarrow T F$ for all $F \in \mathcal{B}$. 
The dense subset we use is the subspace $\mathcal{X}_{0}$ of $\mathcal{X}$ whose elements are compactly supported in $\phi$, namely, given $X \in \mathcal{P}$,

$$
\mathcal{X}_{0}=\left\{F \in \mathcal{X}: \exists R \text { such that }\|F\|_{T_{\phi}}=0 \text { if }\|\phi\|_{\Phi(X \square)} \geq R\right\} .
$$

Lemma B.4. The set $\mathcal{X}_{0}$ is dense in $\mathcal{X}$.

Proof. Let $\chi: \mathbb{C} \rightarrow \mathbb{R}$ be a smooth non-negative function of compact support that is bounded by 1 , equals 1 on a neighbourhood of the unit disk and has support inside the disk of radius 2. For $R \geq 1, x \in Y$, and $\phi \in \Phi$, let $\chi_{R, x}(\phi)=\chi\left(\phi_{x} / R\right)$. Let $F \in \mathcal{X}$ and let $\epsilon>0$. We will show that $R$ can be chosen so that $\left\|F-\chi_{R} F\right\|_{\mathcal{X}}<\epsilon$, and this suffices since $\chi_{R} F \in \mathcal{X}_{0}$.

By the definition of the $T_{\phi}$ norm (see [18, Definition 3.3]), for $R$ large depending on $\mathfrak{h}$,

$$
\left\|\chi_{R, x}\right\|_{T_{\phi}} \leq \sum_{p=0}^{p_{\mathcal{N}}} \frac{1}{p !} \chi^{(p)}\left(\phi_{x} / R\right)(\mathfrak{h} / R)^{p} \leq 1+O(\mathfrak{h} / R) \leq 2 .
$$

Also, since $\left\|1-\chi_{R, x}\right\|_{T_{\phi}}=0$ for $\left|\phi_{x}\right|<R$,

$$
\left\|1-\chi_{R, x}\right\|_{T_{\phi}}=\mathbb{1}_{\left|\phi_{x}\right| \geq R}\left\|1-\chi_{R, x}\right\|_{T_{\phi}} \leq 3 \mathbb{1}_{\|\phi\|_{\Phi} \geq R}
$$

Let $\chi_{R}(\phi)=\prod_{x \in Y} \chi_{R, x}(\phi)$. Let $\succ$ be any total ordering of the points in $Y=X^{\square}$. We apply (B.2) $-(\mathrm{B} .3)$ and the product property of the $T_{\phi}$ semi-norm to obtain

$$
\left\|1-\chi_{R}\right\|_{T_{\phi}} \leq \sum_{y \in Y}\left\|1-\chi_{R, y}\right\|_{T_{\phi}} \prod_{x \in Y, x \succ y}\left\|\chi_{R, x}\right\|_{T_{\phi}} \leq \mathbb{1}_{\|\phi\|_{\Phi(Y)} \geq R} 3|Y| 2^{|Y|} .
$$

By hypothesis, $\|F\|_{T_{\phi}} \mathcal{G}^{-1}(X, \phi) \rightarrow 0$ as $\|\phi\|_{\Phi(Y)} \rightarrow \infty$. By the product property, for $R$ sufficiently large depending on $\epsilon, \mathfrak{h}, Y$, this gives

$$
\left\|F-\chi_{R} F\right\|_{\mathcal{X}} \leq 3|Y| 2^{|Y|} \sup _{\phi:\|\phi\|_{\Phi(Y)} \geq R}\|F\|_{T_{\phi}} \mathcal{G}^{-1}(X, \phi)<\epsilon .
$$

This completes the proof.

\section{B.1.3 Continuity of expectation in covariance}

Before proving Proposition B.1, we first prove the following lemma concerning a norm equivalence. We write $Y=X^{\square}$ below, to simplify the notation. The normed space $\mathcal{X}$ is defined above Proposition B.1.

Lemma B.5. Let $S \subset \mathbb{C}^{Y}$ and let $F_{n} \in \mathcal{X}$ for $n \in \mathbb{N}$. Then $F_{n}$ is convergent in $T_{\phi}$ semi-norm uniformly in $\phi \in S$ if and only if $F_{n, y}$ and its derivatives up to order $p_{\mathcal{N}}$ converge uniformly in $\phi \in S$ for the finitely many possible sequences $y$ arising from $Y$. This is the same as $F_{n, y}$ being convergent in the $C^{p_{\mathcal{N}}}(S)$ topology for each such $y$. 
Proof. The proof is closely related to that of Proposition A.1. Given $\phi \in S$ and $g \in \Phi$, we define the linear functional $\lambda_{\phi, g}: \mathcal{X} \rightarrow \mathbb{C}$ by $F \mapsto\langle F, g\rangle_{\phi}$. Then

$$
\sup _{\phi \in S}\|F\|_{T_{\phi}}=\sup _{\phi \in S, g \in B(\Phi)}\left|\lambda_{\phi, g}(F)\right| \text {. }
$$

Therefore the sequence $F_{n}$ in $\mathcal{S}$ is convergent in $T_{\phi}$ uniformly in $\phi \in S$ if and only if $\lambda_{\phi, g}\left(F_{n}\right)$ is convergent in $\mathbb{C}$, uniformly in the parameters $(\phi, g) \in S \times B(\Phi)$.

Let $z \in \Lambda^{*}$. We define a test function $\delta_{z} \in \Phi$ by setting $\delta_{z}\left(z^{\prime}\right)=1$ when $z^{\prime}=z$ and $\delta_{z}\left(z^{\prime}\right)=0$ otherwise, for $z^{\prime} \in \Lambda^{*}$. All test functions are finite linear combinations of these special test functions and they comprise a finite basis for the test functions in $\Phi(Y)$. Therefore $\lambda_{\phi, g}\left(F_{n}\right)$ is convergent uniformly in $(\phi, g)$ if and only if $\lambda_{\phi, g}\left(F_{n}\right)$ is convergent uniformly in $\phi \in S$ when $g$ is a basis test function. Exactly as in (A.4), $\left\langle F_{n}, \delta_{x, y}\right\rangle_{\phi}$ is a partial derivative of $F_{n, y}$ with respect to $\phi$. Therefore, uniform convergence of $\lambda_{\phi, g}\left(F_{n}\right)$ is equivalent to uniform convergence of partial derivatives, as claimed.

Proof of Proposition B.1. By Proposition [5.3, the linear map $T_{C}: F \mapsto \mathbb{E}_{C} \theta F$ from $\mathcal{X}$ to $\mathcal{X}_{+}$ is a bounded operator. The proof of Proposition 5.3 shows that $\left\|T_{C}\right\|$ is bounded uniformly in $C \in B_{1}\left(\Phi_{+}\right)$. Let $C_{n}$ be a sequence of covariances in $B_{1}(\Phi)$ that converges to $C$, and let $T_{C_{n}}: F \mapsto \mathbb{E}_{C_{n}} \theta F$. By Lemmas B.3 B.4, it suffices to show that $T_{C_{n}} F \rightarrow T_{C} F$ for all $F \in \mathcal{X}_{0}$, with $\mathcal{X}_{0}$ the dense subset of $\mathcal{X}$ defined by (B.1). An element $F \in \mathcal{X}_{0}$ has the form $F=\sum_{y} \frac{1}{y !} F_{y} \psi^{y}$, and this is a finite sum because there are finitely many fermion fields with labels in $Y$. Therefore it suffices to show that, for each finite sequence $y, \mathbb{E}_{C_{n}} \theta F_{y} \psi^{y}$ converges to $\mathbb{E}_{C} \theta F_{y} \psi^{y}$. By [18, (2.39)], $\mathbb{E}_{C} \theta F=\left(\mathbb{E}_{C} \theta F_{y}\right)\left(\mathbb{E}_{C} \theta \psi^{y}\right)$, so it suffices to prove that $\mathbb{E}_{C_{n}} \theta F_{y} \rightarrow \mathbb{E}_{C} \theta F_{y}$ and $\mathbb{E}_{C_{n}} \theta \psi^{y} \rightarrow \mathbb{E}_{C} \theta \psi^{y}$.

Since $F_{y} \psi^{y} \in \mathcal{N}(Y)$ we can regard $C$ as an element of the finite-dimensional vector space $\Phi_{+}(Y)$, on which all norms are equivalent. In particular a sequence $C_{n}$ of covariances converges in $\Phi_{+}(Y)$ if and only if the sequence converges in the sense of convergence of matrix elements of $Y \times Y$ matrices. By definition, $\mathbb{E}_{C} \theta \psi^{y}$ is a polynomial in $\psi_{x}$ for $x \in Y$, with coefficients that are polynomials in matrix elements of $C$. The space of such polynomials is finite-dimensional, so the map $C \mapsto \mathbb{E}_{C} \theta \psi^{y}$ is continuous.

It remains to prove that $C \mapsto \mathbb{E}_{C} \theta F_{y}$ is continuous as a map from a domain of fixed-size matrices to $\mathcal{X}_{+}$, for $F_{y}$ a compactly supported $p_{\mathcal{N}}$ times continuously differentiable function of $\phi \in \mathbb{C}^{Y}$. However, the map $\mathbb{E}_{C} \theta$ represents convolution by a Gaussian, and from this it can be seen that $\mathbb{E}_{C} \theta F_{y}$ is jointly continuous in $\phi$ and $C$. Derivatives commute with convolution so the same is true for derivatives. Therefore, $\mathbb{E}_{C} \theta F_{y}$ is continuous in $C$, as a map into $C^{p_{\mathcal{N}}}\left(\mathbb{C}^{Y}\right)$. By Lemma B.5, it is therefore also continuous in the topology of convergence in $T_{\phi}$ norm uniformly in $\phi$. This is a stronger topology than the norm on $\mathcal{X}_{+}$, so the proof is complete.

\section{B.2 Expectation and vanishing at weighted infinity}

We now prove that the property of vanishing at weighted infinity is preserved by the expectation.

Since we only take expectations in finite volume we consider the vector space $\mathcal{K}(\Lambda)$ with the $\mathcal{F}(\mathcal{G})$ norm defined in (1.41) in terms of the weight

$$
W(X, \phi)=\rho^{f(a, X)} \mathcal{G}(X, \phi)
$$


of (1.40), with $\rho$ given by (1.43). There is also the space $\mathcal{F}_{+}\left(\mathcal{G}_{+}\right)$defined in terms of the weight

$$
W_{+}(X, \phi)=\rho_{+}^{f_{+}\left(a_{+}, X\right)} \mathcal{G}_{+}(X, \phi),
$$

where we assume that $a<a_{+}$. Our norm pairs (1.35) -(1.36) are such that $\mathcal{G}=G_{j}$ is paired with $\mathcal{G}_{+}=T_{0, j+1}$, and $\mathcal{G}_{j}=\tilde{G}_{j}$ is paired with $\mathcal{G}_{+}=\tilde{G}_{j+1}^{\gamma}$. As a first step, we prove the following lemma.

Lemma B.6. Let $f_{R}=\mathbb{1}_{\left\{\|\xi\|_{\Phi(X)} \leq R\right\}}$. Then for $X \in \mathcal{P}$ and $\phi \in \mathbb{C}^{X^{\square}}$,

$$
\begin{gathered}
\mathbb{E}_{+} W(X, \phi+\xi) \leq W_{+}(X, \phi), \\
\lim _{R \rightarrow \infty} \sup _{\phi \in \Phi} \frac{1}{W_{+}(X, \phi)} \mathbb{E}_{+}\left[\left(1-f_{R}(\xi)\right) W(X, \phi+\xi)\right]=0 .
\end{gathered}
$$

Proof. By definition of the regulators, and by the inequality $\|\phi+\xi\|^{2} \leq 2\left(\|\phi\|^{2}+\|\xi\|^{2}\right)$,

$$
\mathcal{G}(X, \phi+\xi) \leq \mathcal{G}^{2}(X, \phi) \mathcal{G}^{2}(X, \xi) \leq \mathcal{G}^{2}(X, \phi) G^{2}(X, \xi) .
$$

Using [20, Lemma 1.2], we obtain

$$
\mathcal{G}(X, \phi+\xi) \leq \mathcal{G}_{+}(X, \phi) G^{2}(X, \xi)
$$

By [20, (1.74)],

$$
\mathbb{E}_{+} G^{2}(X, \xi) \leq 2^{|X|_{j}}
$$

and hence

$$
\mathbb{E}_{+} \mathcal{G}(X, \phi+\xi) \leq 2^{|X|_{j}} \mathcal{G}_{+}(X, \phi) .
$$

By (B.7), (B.14), and the fact that $\rho^{f(a, X)} 2^{|X|_{j}} \leq \rho_{+}^{f_{+}\left(a_{+}, X\right)}$ by definition (for small $\tilde{g}$ ),

$$
\mathbb{E}_{+} W(X, \phi+\xi) \leq \rho^{f(a, X)} 2^{|X|_{j}} \mathcal{G}_{+}(X, \phi) \leq W_{+}(X, \phi) .
$$

This completes the proof of (B.9).

To prove (B.10), we repeat the steps in the proof of $(\overline{B .9})$ but with the factor $1-f_{R}$ included. This factor then appears under the expectation in (B.13), and (B.10) then follows by dominated convergence.

A second ingredient we need is that for a function $f=f(\xi)$ of the fluctuation field $\xi$,

$$
\left\|\mathbb{E}_{+} f F(X)\right\|_{T_{\phi,+}(\mathfrak{h}+)} \leq \mathbb{E}_{+}\left[|f(\xi)|\|F(X)\|_{T_{\phi+\xi}(\mathfrak{h})}\right] .
$$

This follows from a slight adaptation of [20, (7.2)-(7.3)], with the improved version of [18, Proposition 3.19] provided by [18, (3.68)] to include the function $h=f_{R}$. These give the inequality

$$
\left\|\mathbb{E}_{+} f F(X)\right\|_{T_{\phi}(\mathfrak{h} / 2)} \leq \mathbb{E}_{+}\left[|f(\xi)|\|F(X)\|_{T_{\phi+\xi}(\mathfrak{h})}\right] .
$$

With the monotonicity in $\mathfrak{h}$ of the $T_{\phi}$ norm provided by [20, Lemma 3.2], (B.16) then follows from $\mathfrak{h}_{+} \leq \frac{1}{2} \mathfrak{h}$. This application of [20, Lemma 3.2] requires that $F \in \mathcal{N}$ is gauge invariant and such that $\pi_{a b} F=0$ when $j<j_{a b}$, so we make this assumption throughout the rest of the section without further mention. 
Proposition B.7. Suppose $F \in \mathcal{K}$ vanishes at $W$-weighted infinity. Then $\mathbb{E}_{+} \theta F$ vanishes at $W_{+}$-weighted infinity.

Proof. Let $C_{F}=\|F\|_{W}$ and let $X$ be a polymer. Given $R>0$, let $f_{R}=\mathbb{1}_{\left\{\|\xi\|_{\Phi(X)} \leq R\right\}}$. We write $I_{R}=\mathbb{E}_{+} f_{R} \theta F(X)$ and $I_{R}=\mathbb{E}_{+}\left(1-f_{R}\right) \theta F(X)$ so that

$$
\mathbb{E}_{+} \theta F(X)=I_{R}+I_{R} .
$$

By (B.10), we can choose $R$ large such that

$$
\mathbb{E}_{+}\left[\left|1-f_{R}(\xi)\right| W(X, \phi+\xi)\right] \leq C_{F}^{-1} \epsilon W_{+}(X, \phi) .
$$

Therefore, by (B.16),

$$
\begin{aligned}
\left\|I_{\not R}\right\|_{T_{\phi}\left(\mathfrak{h}_{+}\right)} & \leq \mathbb{E}_{+}\left|1-f_{R}(\xi)\right|\|F(X)\|_{T_{\phi+\xi}(\mathfrak{h})} \\
& \leq \mathbb{E}_{+}\left|1-f_{R}(\xi)\right| C_{F} W(X, \phi+\xi) \leq \epsilon W_{+}(X, \phi),
\end{aligned}
$$

and hence

$$
\limsup _{\|\phi\|_{\Phi} \rightarrow \infty} \frac{1}{W_{+}(X, \phi)}\left\|I_{R}\right\|_{T_{\phi}(\mathfrak{h}+)} \leq \epsilon .
$$

Let

$$
M(\phi)=\sup _{\xi}\left|f_{R}(\xi)\right| \frac{1}{W(X, \phi+\xi)}\|F(X)\|_{T_{\phi+\xi}(\mathfrak{h})} .
$$

By (B.16) and (B.9),

$$
\left\|I_{R}\right\|_{T_{\phi}(\mathfrak{h})} \leq \mathbb{E}_{+}\left|f_{R}(\xi)\right|\|F(X)\|_{T_{\phi+\xi}(\mathfrak{h})} \leq M(\phi) \mathbb{E}_{+} W(\phi+\xi) \leq M(\phi) W_{+}(X, \phi) .
$$

When $\|\xi\|_{\Phi} \leq R$, if $\|\phi\|_{\Phi} \rightarrow \infty$ then also $\|\phi+\xi\|_{\Phi} \rightarrow \infty$. Since $F$ vanishes at weighted infinity, it follows that $M(\phi) \rightarrow 0$ as $\|\phi\|_{\Phi} \rightarrow \infty$, and hence

$$
\lim _{\|\phi\|_{\Phi} \rightarrow \infty}\left\|I_{R}\right\|_{T_{\phi}(\mathfrak{h})} W_{+}^{-1}(X, \phi)=0
$$

With (B.21) and (B.18), this concludes the proof.

\section{Polymer geometry}

We now prove some geometric lemmas used in our analysis. They concern $f_{j}(z, a, X)$, which is defined for $z \geq 0$ and $a \in\left(0,2^{-d}\right]$ and $X \in \mathcal{P}_{j}$ by (5.21). We begin with the following elementary but useful observation. We claim that for $X \in \mathcal{P}, 0 \leq a^{\prime}<a, C \geq 1$, and for $\epsilon$ sufficiently small,

$$
C^{|X|_{j}} \epsilon^{f(z, a, X)} \leq C^{2^{d}} \epsilon^{f\left(z, a^{\prime}, X\right)}
$$

This follows from

$$
C^{|X|_{j}} \epsilon^{f(z, a, X)} \leq \epsilon^{z} C^{2^{d}}\left(C \epsilon^{a}\right)^{\left(|X|_{j}-2^{d}\right)_{+}} \leq \epsilon^{z} C^{2^{d}}\left(\epsilon^{a^{\prime}}\right)^{\left(|X|_{j}-2^{d}\right)_{+}}=C^{2^{d}} \epsilon^{f\left(z, a^{\prime}, X\right)},
$$


when $\epsilon$ is small enough that $C \epsilon^{a} \leq \epsilon^{a^{\prime}}$.

The following is a subadditivity property of $f_{j}$. Fix any $a \in\left[0,2^{-d} z\right]$, and let $X=\bigcup_{i} X_{i}$ be a nonempty union of disjoint sets $X_{1}, \ldots X_{n} \in \mathcal{P}_{j}$. Then

$$
f_{j}(z, a, X) \leq \sum_{i} f_{j}\left(z, a, X_{i}\right)
$$

To prove this, we observe that for $|X|_{j} \leq 2^{d}$ the inequality reduces to $z \leq \sum_{i} z$, and otherwise the left-hand side equals

$$
z-a 2^{d}+\sum_{i} a\left|X_{i}\right|_{j} \leq \sum_{i}\left(z-a 2^{d}+a\left|X_{i}\right|_{j}\right) \leq \sum_{i} f_{j}\left(z, a, X_{i}\right) .
$$

For $F, G \in \mathcal{K}_{j}$ it is straightforward to check that $F \circ G$ is in $\mathcal{K}_{j}$. We use the following estimate for the circle product several times.

Lemma C.1. Fix $0<a_{\text {out }}<a_{\text {in }} \in\left(0,2^{-d}\right]$ and let $\bar{\epsilon}$ be sufficiently small depending on $a_{\text {out }}, a_{\text {in }}$. Let $\epsilon \in(0,1)$ and $\delta=2^{-2^{d}} \epsilon$. If $F, G \in B_{\text {in }}(\delta)$ then $F \circ G \in B_{\text {out }}(\epsilon)$.

Proof. By the triangle inequality, the product property of the norm, the hypotheses and the subadditivity (C.3) of $f_{j}$, we have, for $Z$ connected and $\bar{\epsilon} \leq 1$,

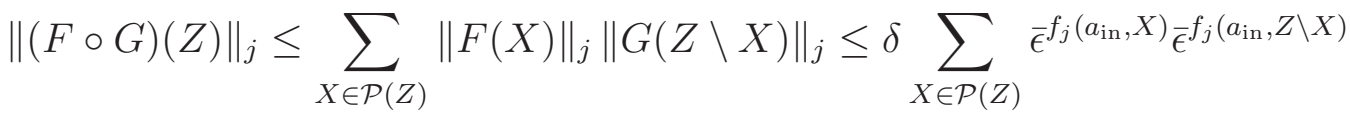

$$
\begin{aligned}
& \leq \delta 2^{|Z|} \bar{\epsilon}^{f_{j}\left(a_{\text {in }}, Z\right)} \leq \delta 2^{2^{d}} \bar{\epsilon}^{f_{j}\left(a_{\text {out }}, Z\right)}=\epsilon \bar{\epsilon}^{f_{j}\left(a_{\text {out }}, Z\right)} .
\end{aligned}
$$

The last inequality is obtained from (C.1) and requires $\bar{\epsilon}$ to be small. This completes the proof.

Lemma C.2. For $z \geq 0, z_{\text {lead }} \geq a \geq 0$, and $X, Y$ disjoint with $X \neq \varnothing$,

$$
f(z, a, X)+z_{\text {lead }}|Y|_{j} \geq f(z, a, X \cup Y) .
$$

Proof. Case $|X|_{j} \geq 2^{d}$. The left-hand side equals

$$
z+a\left(|X|_{j}-2^{d}\right)+z_{\text {lead }}|Y|_{j} \geq z+a\left(|X \cup Y|_{j}-2^{d}\right),
$$

which equals the desired right-hand side.

Case $|X|_{j}<2^{d},|X \cup Y|_{j} \geq 2^{d}$. Since $X$ is not empty the left-hand side equals

$$
z+z_{\text {lead }}|Y|_{j}>z+a|Y|_{j}+a\left(|X|_{j}-2^{d}\right)=z+a\left(|X \cup Y|_{j}-2^{d}\right),
$$

which equals the desired right-hand side.

Remaining case $|X \cup Y|_{j}<2^{d}$. Since $X$ is not empty, the left-hand side equals

$$
z+z_{\text {lead }}|Y|_{j} \geq z=z+a\left(|X \cup Y|_{j}-2^{d}\right)_{+},
$$

which equals the desired right-hand side. 
The following lemma is stated (but not proved) above [24, Lemma 2]. A consequence of the lemma is that if $X \in \mathcal{S}_{j}$ then $\bar{X} \in \mathcal{S}_{j+1}$. The important geometrical constant $\eta=\eta(d)>1$ used in Lemma 5.6 is introduced in Lemma C.3.

Lemma C.3. There is an $\eta=\eta(d)>1$ such that for all $L \geq L_{0}(d)=2^{d}+1$ and for all large sets $X \in \mathcal{C}_{j}$,

$$
|X|_{j} \geq \eta|\bar{X}|_{j+1}
$$

In addition, (C.11) holds with $\eta=1$ for all $X \in \mathcal{P}_{j}$ (not necessarily connected, and possibly small).

Proof. Fix $L \geq L_{0}(d)=2^{d}+1$ (this restriction enters only in the third paragraph of the proof). It is clear that for any $m \geq 1$ the closure of any set of $m j$-blocks contains at most $m(j+1)$-blocks, and hence (C.11) always holds with $\eta=1$.

Assume that $X$ is a large connected set. Let $\Delta=\Delta(d)$ denote the maximum possible number of blocks that touch a connected set of $2^{d}+1$ blocks. We will prove (C.11) by induction on $|\bar{X}|_{j+1}$, with $\eta=1+1 /\left(2^{d}+1+2^{d} \Delta\right)$.

To begin the induction, we claim that if $|\bar{X}|_{j+1}=2^{d}+1$ then $|X|_{j} \geq 2^{d}+2$, and hence

$$
\frac{|X|_{j}}{|\bar{X}|_{j+1}} \geq \frac{2^{d}+2}{2^{d}+1}=1+\frac{1}{2^{d}+1} \geq \eta
$$

To prove the claim, we proceed as follows. The maximum possible value of $|\bar{X}|_{j+1}$ is $|X|_{j}$, so we only need to rule out the case $|X|_{j}=|\bar{X}|_{j+1}=2^{d}+1$, which we now assume. Let $D(X)$ be the integer part of $L^{-j} \max _{x, y \in X}|x-y|_{\infty}$; this is a measure of the diameter of $X$ counted in number of $j$-blocks. Then $D(X) \leq 2^{d}+1 \leq L$. Also, every $j$-block in $X$ lies in a different $(j+1)$-block in $\bar{X}$. However, any set of $2^{d}+1(j+1)$-blocks contains a pair of blocks $B_{1}, B_{2}$ that do not touch. Therefore $D\left(b_{1} \cup b_{2}\right)>L$ for every pair of $j$-blocks $b_{1} \in B_{1}$ and $b_{2} \in B_{2}$, so that $b_{1} \cup b_{2} \subset X$ is not possible. This contradiction proves the claim.

To advance the induction, suppose that (C.11) holds when $2^{d}+1 \leq|\bar{X}|_{j+1} \leq n$, and suppose now that $|\bar{X}|_{j+1}=n+1$. We remove from $\bar{X}$ a connected subset of $2^{d}+1$ blocks. The complement of this connected subset consists of no more than $\Delta$ connected components (since if there were more then one of these components is not adjacent to the removed subset nor to any of the at most $\Delta$ components adjacent to the removed subset, and hence $X$ would be disconnected). We list these components as $\bar{X}_{1}, \ldots, \bar{X}_{\Delta}$, and choose $k \in\{0,1, \ldots, \Delta\}$ such that $\left|\bar{X}_{i}\right|_{j+1} \geq 2^{d}+1$ for $i \leq k$ and $\left|\bar{X}_{i}\right|_{j+1} \leq 2^{d}$ for $i>k$ (some of the latter components may be empty). Let $M=\sum_{i=1}^{k}\left|\bar{X}_{i}\right|_{j+1}$ and $m=\sum_{i=k+1}^{\Delta}\left|\bar{X}_{i}\right|_{j+1}$. By the induction hypothesis applied to $\bar{X}_{i}$ for $i \leq k$, and by (C.11) with $\eta=1$ for $i>k$,

$$
\begin{aligned}
\frac{|X|_{j}}{|\bar{X}|_{j+1}} & \geq \frac{2^{d}+2+\eta M+m}{2^{d}+1+M+m}=1+\frac{1+(\eta-1) M}{2^{d}+1+M+m} \\
& \geq 1+\frac{1+(\eta-1) M}{2^{d}+1+M+\Delta 2^{d}}=1+\frac{1+(\eta-1) M}{\frac{1}{\eta-1}+M}=\eta,
\end{aligned}
$$

where we used our specific choice for the value of $\eta$ in the penultimate step (note that the last equality is true no matter what the value of $M$ ). This advances the induction and completes the proof. 
Lemma C.4. Suppose that either $X_{K}$ has at least two components, or $X_{K}$ has at least one component and $X_{\delta I} \neq \varnothing$. Let $n_{\delta I}=\left|X_{\delta I}\right|_{j}$ and write $X_{K_{i}}$ for the connected components of $X_{K}$. Let $z \geq z_{0}>0$. Let $0<a \leq 1$ and let $\tilde{a} \in(a, \eta a)$. There exist positive $\delta, v$, depending on $d, z_{0}, \tilde{a}, a$, such that

$$
n_{\delta I}+\sum_{i} f_{j}\left(z, a, X_{K_{i}}\right) \geq v+\delta\left|\overline{X_{\delta I} \cup X_{K}}\right|_{j+1}+f_{j+1}\left(z, \tilde{a}, \overline{X_{\delta I} \cup X_{K}}\right) .
$$

Proof. Suppose first that $\overline{X_{\delta I} \cup X_{K}} \in \mathcal{S}_{j+1}$. Then the right-hand side is at most $v+\delta 2^{d}+z$. In the two cases listed at the beginning of the statement of the lemma, the left-hand side is at least $2 z, 1+z$. There exist $v, \delta$ positive so that each of these is greater than $v+\delta 2^{d}+z$.

So suppose now that $\overline{X_{\delta I} \cup X_{K}} \notin \mathcal{S}_{j+1}$. For non-empty $X_{K}$ we let $\sum_{i}$ denote the sum over components $X_{K_{i}}$. We reduce $v, \delta$, if necessary, so that $\tilde{a}+\delta \leq \eta a$ and $v-\tilde{a} 2^{d} \leq-a 2^{d}$. By Lemma C.3, using $a \leq 1$ and (C.3), we have

$$
\begin{aligned}
v+\delta\left|\overline{X_{\delta I} \cup X_{K}}\right|_{j+1}+f_{j+1}\left(z, \tilde{a}, \overline{X_{\delta I} \cup X_{K}}\right) & \leq v+z-\tilde{a} 2^{d}+\eta a\left|\overline{X_{\delta I} \cup X_{K}}\right|_{j+1} \\
& \leq v+z-\tilde{a} 2^{d}+a n_{\delta I}+a\left|X_{K}\right|_{j} \\
& \leq z-a 2^{d}+n_{\delta I}+a\left|X_{K}\right|_{j} \\
& \leq n_{\delta I}+f_{j}\left(z, a, X_{K}\right) \\
& \leq n_{\delta I}+\sum_{i} f_{j}\left(z, a, X_{K_{i}}\right),
\end{aligned}
$$

as required.

Lemma C.5. Let $0<z<2 z^{\prime}$. Recall the definition of $\mathcal{Y}_{0}(W)$ below (D.17). There exists $c=c(d)$ such that for $a_{\text {in }} \in(0, c), a_{\text {out }} \in\left[0, a_{\text {in }}\right]$, and for $\left(X,\left\{\left(U_{B}, B\right)\right\}, U_{M}\right) \in \mathcal{Y}_{0}(W)$,

$$
z^{\prime}|X|_{j}+\sum_{i} f_{j}\left(z, a_{\text {in }}, U_{M, i}\right) \geq\left(a_{\text {in }}-a_{\text {out }}\right)|W|_{j}+f_{j}\left(z, a_{\text {out }}, W\right) .
$$

Proof. Let $U_{M, i}, i=1, \ldots, n_{M}$, be the components of $U_{M}$. Let $S$ denote the number of small sets $U$ that can contain a given block $B$. Then $\left|X^{\square}\right|_{j} \leq 2^{d} S|X|_{j}$, and hence, since $W=X^{\square} \cup U_{M}$,

$$
|W|_{j} \leq 2^{d} S|X|_{j}+\sum_{i}\left|U_{M, i}\right|_{j}
$$

Letting $u=a_{\text {in }}-a_{\text {out }}$ we rewrite this as

$$
u|W|_{j}+a_{\text {out }}|W|_{j}+z-a_{\text {out }} 2^{d} \leq a_{\text {in }} 2^{d} S|X|_{j}+\sum_{i} a_{\text {in }}\left|U_{M, i}\right|_{j}+z-a_{\text {out }} 2^{d} .
$$

The definition of $\mathcal{Y}_{0}$ excludes the case $X=\varnothing$ so we assume $X \neq \varnothing$ and we can also assume $W \notin \mathcal{S}$, because $W=X^{\square} \cup U_{M}$ and $X^{\square} \notin \mathcal{S}$. Then $f_{j}\left(z, a_{\text {out }}, W\right)=z-a_{\text {out }} 2^{d}+a_{\text {out }}|W|_{j}$. Therefore the left-hand side is $u|W|_{j}+f_{j}\left(z, a_{\text {out }}, W\right)$. Let $v=z-a_{\text {in }} 2^{d}$ so that $v+a_{\text {in }}|U|_{j} \leq f_{j}\left(z, a_{\text {in }}, U\right)$. Then 
we can rewrite the inequality as

$$
\begin{aligned}
u|W|_{j}+f_{j}\left(z, a_{\text {out }}, W\right) & \leq a_{\mathrm{in}} 2^{d} S|X|_{j}+\sum_{i} a_{\text {in }}\left|U_{M, i}\right|_{j}+v+u 2^{d} \\
& =a_{\mathrm{in}} 2^{d} S|X|_{j}+\sum_{i}\left(v+a_{\mathrm{in}}\left|U_{M, i}\right|_{j}\right)+\left(1-n_{M}\right) v+u 2^{d} \\
& \leq a_{\mathrm{in}} 2^{d} S|X|_{j}+\sum_{i} f_{j}\left(z, a_{\mathrm{in}}, U_{M, i}\right)+\left(1-n_{M}\right) v+u 2^{d} .
\end{aligned}
$$

We choose $a_{\text {in }}>0$ sufficiently small that $v=z-a_{\text {in }} 2^{d} \geq 0$. Decreasing $a_{\text {in }}$ if necessary we have $a_{\text {in }} 2^{d} S+u 2^{d} \leq z^{\prime}$. If $n_{M} \geq 1$ then we use $a_{\text {in }} 2^{d} S|X|_{j}+u 2^{d} \leq z^{\prime}|X|_{j}$ to obtain the desired result.

Now we consider the case $n_{M}=0$, which is the same as $U_{M}=\varnothing$. Decreasing $a_{\text {in }}$ if necessary, and using $z<2 z^{\prime}$, we have $a_{\text {in }} 2^{d} S+\frac{1}{2} z+u 2^{d} \leq z^{\prime}$. The definition of $\mathcal{Y}_{0}$ requires $|X|_{j} \geq 2$ when $U_{M}=\varnothing \mathrm{so}$

$$
\begin{aligned}
a_{\text {in }} 2^{d} S|X|_{j}+\left(1-n_{M}\right) v+u 2^{d} & =a_{\text {in }} 2^{d} S|X|_{j}+v+u 2^{d} \\
& \leq a_{\text {in }} 2^{d} S|X|_{j}+z+u 2^{d} \\
& \leq\left(a_{\text {in }} 2^{d} S+\frac{1}{2} z+u 2^{d}\right)|X|_{j} \\
& \leq z^{\prime}|X|_{j} .
\end{aligned}
$$

This completes the proof.

\section{Change of variables}

In this section, we prove Proposition 4.1, which for convenience we restate here as Proposition D.1. For further discussion of this proposition, see [14, Section 5]. This section applies for any norm $\|\cdot\|$ on $\mathcal{N}$ which obeys the product property [20, (1.44)]. We make use of (4.1)-(4.5), and in particular recall that $M$ is defined in (4.5), for $K_{\text {in }} \in \mathcal{K}$ and $U \in \mathcal{C}$ and with $\bar{J}(U, B)=I_{\text {in }}^{U} J(U, B)$, by

$$
M(U)=K_{\text {in }}(U)-\sum_{B \in \mathcal{B}(U)} \bar{J}(U, B) .
$$

Proposition D.1. Let $a_{\mathrm{in}}$ be small as specified in Lemma C.5. Let $a_{\mathrm{out}}<a_{\text {in }}$ and $z^{\prime}>\frac{1}{2} z$. Let $\rho$ be sufficiently small depending on the difference $a_{\text {out }}-a_{\text {in }}$. Let $\epsilon \in(0,1)$. Let $J, I_{\text {in }}$ be as in (4.1) - (4.4). Suppose that $K_{\mathrm{in}} \in \mathcal{K}$ and $J$ satisfy

$$
\begin{aligned}
& \sup _{\mathcal{D}(J)}\left\|I_{\text {in }}^{U} J(U, B)\right\| \leq \epsilon \rho^{z^{\prime}}, \\
& M \in B_{\mathcal{F}_{\text {in }}}\left(\epsilon \rho^{z}\right) .
\end{aligned}
$$

Then there exists $K_{\text {out }} \in \mathcal{K}$ such that

$$
\begin{aligned}
& \left(I_{\text {in }} \circ K_{\text {out }}\right)(\Lambda)=\left(I_{\text {in }} \circ K_{\text {in }}\right)(\Lambda), \\
& K_{\text {out }} \text { is polynomial in } I_{\mathrm{in}}, \bar{J}, K_{\text {in }}, \\
& K_{\text {out }}=M+E \text { with } E \in B_{\mathcal{F}_{\text {out }}}\left(\epsilon \rho^{z+\left(a_{\text {in }}-a_{\text {out }}\right) / 2}\right) .
\end{aligned}
$$

If $K_{\mathrm{in}}=0$ and $J=0$, then $K_{\text {out }}=0$. 


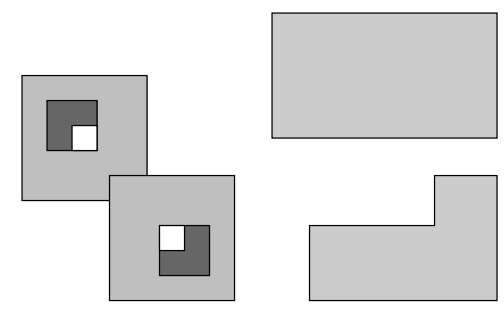

Figure 3: This figure illustrates an element of $\mathcal{Y}(W)$. The white squares are the blocks of $X$, and are centred in two larger squares whose union is $X^{\square}$. Each white square $B$ is contained in a small set $U_{B}$, which is itself contained in $B^{\square}$. The components of $U_{M}$ are the two shaded components without white squares, and $W$ is the total area.

Proof. For $U \in \mathcal{C}$ and $B \in \mathcal{B}$, with $\bar{J}(U, B)=I_{\text {in }}^{U} J(U, B)$, let

$$
\bar{J}(U)=\sum_{B \in \mathcal{B}(U)} \bar{J}(U, B) .
$$

For $U_{J} \in \mathcal{P}$, let

$$
\bar{J}\left(U_{J}\right)=\prod_{U \in \operatorname{Comp}\left(U_{J}\right)} \bar{J}(U) .
$$

By the definition of $M$ and the component factorisation property of $K_{\text {in }}$,

$$
\begin{aligned}
\left(I_{\mathrm{in}} \circ K_{\mathrm{in}}\right)(\Lambda) & =\sum_{U_{\mathrm{in}} \in \mathcal{P}} I_{\mathrm{in}}^{\Lambda \backslash U_{\mathrm{in}}} K_{\mathrm{in}}\left(U_{\mathrm{in}}\right) \\
& =\sum_{U_{\mathrm{in} \in \mathcal{P}}} I_{\mathrm{in}}^{\Lambda \backslash U_{\mathrm{in}}} \prod_{U \in \operatorname{Comp}\left(U_{\mathrm{in}}\right)}(\bar{J}(U)+M(U)) \\
& =\sum_{U_{\mathrm{in} \in \mathcal{P}}} I_{\mathrm{in}}^{\Lambda \backslash U_{\mathrm{in}}} \sum_{\hat{U}_{M} \subset \operatorname{Comp}\left(U_{\mathrm{in}}\right)} \bar{J}\left(U_{\mathrm{in}} \backslash U_{M}\right) M\left(U_{M}\right),
\end{aligned}
$$

where $U_{M}$ is the union of components in $\hat{U}_{M}$.

Given $X \in \mathcal{P}$, let $B_{1}, \ldots, B_{n}$ be a list of the blocks in $\mathcal{B}(X)$, and let

$$
\begin{aligned}
\mathcal{U}(X)= & \left\{\left\{\left(U_{B_{1}}, B_{1}\right), \ldots,\left(U_{B_{n}}, B_{n}\right)\right\}:\right. \\
& \left.U_{B_{i}} \in \mathcal{P}, U_{B_{i}} \supset B_{i}, U_{B_{i}} \text { does not touch } U_{B_{j}} \text { for } i \neq j\right\} .
\end{aligned}
$$

(In particular, $\mathcal{U}(X)$ is empty if any two blocks of $X$ touch each other.) Given an element of $\mathcal{U}(X)$, we write $U_{J}=\cup_{B \in \mathcal{B}(X)} U_{B}$, and write $\mathcal{P}^{\prime}\left(\Lambda \backslash U_{J}\right)$ for the set of polymers that do not touch $U_{J}$. By interchanging the sums over blocks $B$ in (D.7) and polymers $U_{B}$, we obtain

$$
\begin{array}{r}
\left(I_{\text {in }} \circ K_{\text {in }}\right)(\Lambda)=\sum_{X \in \mathcal{P}(\Lambda)} \sum_{\left\{\left(U_{B}, B\right)\right\} \in \mathcal{U}(X)}\left(\prod_{B \in \mathcal{B}(X)} \bar{J}\left(U_{B}, B\right)\right) \\
\times \sum_{U_{M} \in \mathcal{P}^{\prime}\left(\Lambda \backslash U_{J}\right)} M\left(U_{M}\right) I_{\text {in }}^{\Lambda \backslash\left(U_{M} \cup U_{J}\right)} .
\end{array}
$$


Recall the definition of the small set neighbourhood of $X$ in Definition 1.6. For $W=X^{\square} \cup U_{M}$, we write

$$
I_{\text {in }}^{\Lambda \backslash\left(U_{M} \cup U_{J}\right)}=I_{\text {in }}^{\Lambda \backslash W} I_{\text {in }}^{W \backslash\left(U_{M} \cup U_{J}\right)} .
$$

With this notation the claim (D.4) holds with $K_{\text {out }}$ given by

$$
K_{\text {out }}(W)=\sum_{\left(X,\left\{\left(U_{B}, B\right)\right\}, U_{M}\right) \in \mathcal{Y}(W)}\left(\prod_{B \in \mathcal{B}(X)} \bar{J}\left(U_{B}, B\right)\right) M\left(U_{M}\right) I_{\text {in }}^{W \backslash\left(U_{M} \cup U_{J}\right)} \quad(W \in \mathcal{P}) .
$$

Here $\mathcal{Y}(W)$ denotes the set of triples $\left(X,\left\{\left(U_{B}, B\right)\right\}, U_{M}\right)$, with $X \in \mathcal{P}(W),\left\{\left(U_{B}, B\right)\right\} \in \mathcal{U}(X)$, $U_{M} \in \mathcal{P}^{\prime}\left(\Lambda \backslash U_{J}\right)$, and $X^{\square} \cup U_{M}=W$. Note that the small set neighbourhood $X^{\square}$ contains all possible unions $U_{J}$ of small sets in the summation over the $U_{B}$.

Note that the above formula implies that if $J=0$ then $K_{\text {out }}=K_{\text {in }}$. In particular, as claimed, $K_{\text {out }}=0$ when $K_{\text {in }}=0$ and $J=0$. Also, it shows that $K_{\text {out }}$ is polynomial in $I_{\text {in }}, \bar{J}, K_{\text {in }}$ as claimed in (D.5). As an explicit example, for the case where $W$ is a single block $B$, (D.13) gives

$$
K_{\text {out }}(B)=M(B)+\sum_{U:(U, B) \in \mathcal{D}(J)} \bar{J}(U, B)
$$

The properties that define $\mathcal{Y}(W)$, together with the hypothesis that $K_{\text {in }} \in \mathcal{K}$ and that $J$ obeys (4.3), can be used to verify the claim that $K_{\text {out }} \in \mathcal{K}$. In particular, $K_{\text {out }}$ obeys the factorisation property of Definition 1.7 by construction, and the field locality property holds because we have constructed $K_{\text {out }}(W)$ as a polynomial in the local objects $\bar{J}, M, I_{\text {in }}$ evaluated on sets contained in $W$.

For the bound claimed in (D.6), we first show that the contribution to (D.13) due to triples with $|X|=1$ and $U_{M}=\varnothing$ vanishes. This feature is a crucial ingredient. In this case, $X$ is a single block $B, K_{\text {out }}(W)=0$ unless $W=X^{\square}=B^{\square}$ and thus $B$ is uniquely determined by $W$, and by (4.2), the contribution to (D.13) is

$$
\sum_{U \in \mathcal{S}: U \supset B} J(U, B) I_{\mathrm{in}}^{B^{\square}}=0
$$

Let $W \in \mathcal{C}$. As in (5.21), we write

$$
f_{j}(z, a, X)= \begin{cases}z+f_{j}(a, X) & X \neq \varnothing \\ 0 & X=\varnothing\end{cases}
$$

for $z \geq 0, a \in\left(0,2^{-d}\right]$ and $X \in \mathcal{P}_{j}$. We apply the triangle inequality, product property [20, (1.44)], and the hypotheses to (D.13), to obtain

$$
\left\|K_{\text {out }}(W)-M(W)\right\| \leq \sum_{\left(X, \vec{U}, U_{M}\right) \in \mathcal{Y}_{0}(W)} \epsilon \rho^{z^{\prime}|X|} \prod_{i}\left(\rho^{f\left(z, a_{\mathrm{in}}, U_{M, i}\right)}\right) \alpha_{I}^{\left|W \backslash\left(U_{J} \cup U_{M}\right)\right|},
$$

where $\mathcal{Y}_{0}(W)$ imposes the constraints on $\left(X,\left\{\left(U_{B}, B\right)\right\}, U_{M}\right)$ required in (D.13) with the additional constraint that the terms with $X=\varnothing, U_{M}=W$, and with $|X|=1, U_{M}=\varnothing$ are omitted (the first omission is because $M$ is subtracted in (D.17) and the second is due to the cancellation in (D.15) ). 
Since $\alpha_{I} \geq 1$, and since $W \backslash\left(U_{J} \cup U_{M}\right) \subset X^{\square}$ and $\left|X^{\square}\right| \leq 2^{d} S|X|$, the power of $\alpha_{I}$ above can be replaced by const ${ }^{|W|}$. By Lemma C.5, for $\left(X,\left\{\left(U_{B}, B\right)\right\}, U_{M}\right) \in \mathcal{Y}_{0}(W)$, and with $2 u=a_{\text {in }}-a_{\text {out }}$, we have

$$
z^{\prime}|X|+\sum_{i} f\left(z, a_{\mathrm{in}}, U_{M, i}\right) \geq 2 u|W|+f\left(z, a_{\text {out }}, W\right)
$$

Therefore,

$$
\left\|K_{\text {out }}(W)-M(W)\right\| \leq \epsilon \rho^{2 u|W|+f\left(z, a_{\text {out }}, W\right)} \operatorname{const}^{|W|}\left|\mathcal{Y}_{0}(W)\right|
$$

where $\left|\mathcal{Y}_{0}(W)\right|$ denotes the cardinality of $\mathcal{Y}_{0}(W)$. For fixed $X$, there are at most $S^{|X|}$ possible choices of the small sets $U_{B}$ specified in the definition of $\mathcal{Y}_{0}(W)$. We use this, and also relax the summation to disjoint $X$ and $U_{M}$ in $W$. Since there are at most $3^{|W|}$ ways to partition $W$ into $X, U_{M}, W \backslash\left(X \cup U_{M}\right)$, we can absorb $\left|\mathcal{Y}_{0}(W)\right|$ into const $^{|W|}$. Finally, we choose $\rho$ sufficiently small depending on $u$ so that const ${ }^{|W|} \leq \frac{1}{2} \rho^{-u|W|}$. Then

$$
\left\|K_{\text {out }}(W)-M(W)\right\| \leq \frac{1}{2} \epsilon \rho^{u|W|+f\left(z, a_{\text {out }}, W\right)} \leq \frac{1}{2} \epsilon \rho^{u+f\left(z, a_{\text {out }}, W\right)},
$$

which implies (D.6).

\section{E Approximate isometry between finite and infinite vol- ume}

The proof of Theorem 2.5 uses Lemmas E.4 E.6, which are given below. Lemmas E.1E.3 are used in the proof of Lemmas E.4 E.5. In this section $k$ is any scale, in particular it can be $j$ or $j+1$. Let $X \in \mathcal{P}_{k}\left(\mathbb{Z}^{d}\right)$. A coordinate map $\iota$ from $X$ to a torus $\Lambda$ exists for all $\Lambda$ with $\operatorname{diam}(\Lambda) \geq 2 \operatorname{diam}(X)$. Given a coordinate map $\iota: X \rightarrow \Lambda$ and a test function $g \in \Phi(\iota X)$, we define a test function $g_{\iota} \in \Phi(X)$ by $\left(g_{\iota}\right)_{z}=g_{\iota z}$, where $\iota z$ is defined by letting $\iota$ act on the sequence $z$ componentwise.

For the first lemma, recall the pairing in the definition of the $T_{\phi}$ semi-norm in [18, Definition 3.3].

Lemma E.1. Let $X \in \mathcal{P}_{k}\left(\mathbb{Z}^{d}\right)$. For a coordinate map $\iota: X \rightarrow \Lambda, F \in \mathcal{N}(X), g \in \Phi(\Lambda)$, and $\phi \in \mathbb{C}^{\Lambda}$,

$$
\langle\iota F, g\rangle_{\phi}=\left\langle F, g_{\iota}\right\rangle_{\phi_{\iota}}
$$

Proof. Both sides are linear in $F$. By (1.81), it suffices to consider $F=F_{y} \psi^{y}$. Then $\iota F=\iota\left(F_{y}\right) \psi_{\iota}^{y}$. According to (1.82),

$$
\langle\iota F, g\rangle_{\phi}=\sum_{x \in(\iota X)^{*}} \frac{1}{x !}\left(F_{y}\left(\phi_{\iota}\right)\right)_{x} g_{x, \iota y}=\sum_{x \in X^{*}} \frac{1}{x !} F_{x, y}\left(\phi_{\iota}\right) g_{\iota x, \iota y}=\left\langle F, g_{\iota}\right\rangle_{\phi_{\iota}} .
$$

For real $t>0$ and a nonempty polymer $X \in \mathcal{P}_{k}\left(\mathbb{Z}^{d}\right)$, let $X_{t} \subset \mathbb{Z}^{d}$ be the smallest subset that contains $X$ and all points in $\mathbb{Z}^{d}$ that are within distance $t L^{k}$ of $X$. The next lemma expresses a sense in which coordinate maps are approximately isometries as maps between spaces of test functions. Our norms on test functions (see [18, Example 3.2] and [18, (3.37)]) depend on a parameter $\mathfrak{h}$. For the next lemma, we exhibit this dependence by writing $\Phi_{k}(\iota X, \mathfrak{h})$, etc. 
Lemma E.2. Let $X \in \mathcal{P}_{k}\left(\mathbb{Z}^{d}\right)$, let $s>0$, and let $\iota$ be a coordinate map from a polymer containing $X_{s}$ into a torus $\Lambda$. There exists $\mathfrak{h}_{s}>0$ and $c>0$ (independent of $X, k, \iota, \Lambda$ ), with $1 \leq \mathfrak{h} / \mathfrak{h}_{s} \leq$ $1+c s^{-1}$, such that for $g \in \Phi_{k}(\iota X, \mathfrak{h})$,

$$
\left\|g_{\iota}\right\|_{\Phi_{k}(X, \mathfrak{h})} \leq\|g\|_{\Phi_{k}(\iota X, \mathfrak{h})} \leq\left\|g_{\iota}\right\|_{\Phi_{k}\left(X, \mathfrak{h}_{s}\right)},
$$

and likewise for the $\tilde{\Phi}_{k}$ semi-norm.

Proof. We give the proof for the case $\boldsymbol{\Lambda}=\Lambda$, because the general case is merely an elaboration of notation. We write $\Phi=\Phi_{k}$. By the definition of the norm on test functions, we see that it is sufficient to fix an integer $p \geq 1$ and prove the lemma for the case where the test function $g \in \Phi(\iota X, \mathfrak{h})$ is zero except on sequences of length $p$. The domain $\iota X$ is contained in a torus $\Lambda$. By thinking of $\Lambda$ as a hypercube in a lattice of hypercubes paving $\mathbb{Z}^{d}$, we identify a test function on $\Lambda$ with a function on $\left(\mathbb{Z}^{d}\right)^{p}$ which is periodic in each component. The $\Phi(\iota X, \mathfrak{h})$ norm of $g$ is the infimum of $\left\|g^{\prime}\right\|_{\Phi(\Lambda)}$ over extensions $g^{\prime}$ of $g$ to $\Lambda^{p}$; by the identification and the definition of $g_{\iota}$ this is the infimum of $\left\|g_{\iota}^{\prime}\right\|_{\Phi\left(\mathbb{Z}^{d}, \mathfrak{h}\right)}$ over extensions $g_{\iota}^{\prime}$ of $g_{\iota}$ to functions of $\left(\mathbb{Z}^{d}\right)^{p}$ that are periodic in each component. The norm $\left\|g_{\iota}\right\|_{\Phi(X, \mathfrak{h})}$ is the same but the extensions are not constrained to be periodic. Therefore

$$
\|g\|_{\Phi(\iota X, \mathfrak{h})} \geq\left\|g_{\iota}\right\|_{\Phi(X, \mathfrak{h})}
$$

which is the lower bound claimed in (E.3).

Let $r=\frac{s}{3}$. By the definition of $\Phi(X, \mathfrak{h})$, there exists an extension $\tilde{g}_{\iota} \in \Phi\left(\mathbb{Z}^{d}, \mathfrak{h}\right)$ of $g_{\iota}$ such that

$$
\left\|\tilde{g}_{\iota}\right\|_{\Phi\left(\mathbb{Z}^{d}, \mathfrak{h}\right)} \leq\left(1+r^{-1}\right)\left\|g_{\iota}\right\|_{\Phi(X, \mathfrak{h})} .
$$

By [19, Lemma 3.3], there exists a function $\chi=\chi_{r}$, which is equal to 1 on $X^{p}$ and 0 on $\mathbb{Z}^{d p} \backslash X_{2 r}^{p}$, and a constant $c_{0}>0$ (independent of $p, X$, and $L^{j}$ ), such that

$$
\left\|\tilde{g}_{\iota} \chi\right\|_{\Phi\left(\mathbb{Z}^{d}, \mathfrak{h}\right)} \leq\left(1+c_{0} r^{-1}\right)^{p}\left\|\tilde{g}_{\iota}\right\|_{\Phi\left(\mathbb{Z}^{d}, \mathfrak{h}\right)} .
$$

In combination with (E.5), this gives the existence of $\mathfrak{h}_{s}$ obeying the desired bound, such that

$$
\left\|\tilde{g}_{\iota} \chi\right\|_{\Phi\left(\mathbb{Z}^{d}, \mathfrak{h}\right)} \leq\left\|g_{\iota}\right\|_{\Phi\left(X, \mathfrak{h}_{s}\right)} .
$$

By hypothesis, the domain of $\iota$ strictly contains $X_{2 r}$, so we can invert $\iota$ on $\iota X_{2 r}$. Therefore $\left(\tilde{g}_{\iota} \chi\right)_{\iota^{-1}}$ is an extension of $\left.g\right|_{\iota X}$ to the subset $\iota X_{2 r}$ of $\Lambda$. Provided $L$ is large enough so that $r L^{k} \geq p_{\Phi}$ for all $k$, the derivatives up to order $p_{\Phi}$ in each argument of this extension are zero near the inner boundary of $\iota X_{2 r}$ so we can further extend by zero to all of $\Lambda$. Call this extension $G$. Then, by definition of $\Phi(\iota X, \mathfrak{h})$,

$$
\|g\|_{\Phi(\iota X, \mathfrak{h})} \leq\|G\|_{\Phi(\Lambda, \mathfrak{h})}=\left\|\tilde{g}_{\iota} \chi\right\|_{\Phi\left(\mathbb{Z}^{d}, \mathfrak{h}\right)} \leq\left\|g_{\iota}\right\|_{\Phi\left(X, \mathfrak{h}_{s}\right)}
$$

and this proves the upper bound of (E.3).

The next three lemmas express senses in which coordinate maps are isometries, provided a small change is made in the parameter $\mathfrak{h}$. 
Lemma E.3. Let $X \in \mathcal{P}_{k}\left(\mathbb{Z}^{d}\right)$, let $\iota: \tilde{X} \rightarrow \Lambda$ be a coordinate map with $X_{N / 4} \subset \tilde{X} \in \mathcal{P}_{k}\left(\mathbb{Z}^{d}\right)$, and let $\phi \in \mathbb{C}^{\Lambda}$. The induced map $\iota: \mathcal{N}(X) \rightarrow \mathcal{N}(\iota X)$ is defined in (1.82). This map is linear, and there exists $\mathfrak{h}^{-}=\mathfrak{h}^{-}(N) \leq \mathfrak{h}$, with $\mathfrak{h}^{-} \rightarrow \mathfrak{h}$ as $N=N(\Lambda) \rightarrow \infty$, such that $\|F\|_{T_{\phi_{\iota}}\left(\mathfrak{h}^{-}\right)} \leq$ $\|\iota F\|_{T_{\phi}(\mathfrak{h})} \leq\|F\|_{T_{\phi_{\iota}}(\mathfrak{h})}$ for all $F \in \mathcal{N}(X)$.

Proof. The linearity of the map is clear. We write $\Phi=\Phi_{k}$. Let $F \in \mathcal{N}(X)$ and $g \in \Phi(\iota X, \mathfrak{h})$. By Lemma E.1, the definition of the $T_{\phi}(\mathfrak{h})$ norm, and Lemma E.2.

$$
\left|\langle\iota F, g\rangle_{\phi}\right|=\left|\left\langle F, g_{\iota}\right\rangle_{\phi_{\iota}}\right| \leq\|F\|_{T_{\phi_{\iota}}(\mathfrak{h})}\left\|g_{\iota}\right\|_{\Phi(X, \mathfrak{h})} \leq\|F\|_{T_{\phi_{\iota}(\mathfrak{h})}}\|g\|_{\Phi(\iota X, \mathfrak{h})}
$$

Taking the supremum over $g$ with unit norm, we have $\|\iota F\|_{T_{\phi}(\mathfrak{h})} \leq\|F\|_{T_{\phi_{\iota}}(\mathfrak{h})}$ which is one of the desired inequalities. For the reverse estimate, we consider $N(\Lambda) \rightarrow \infty$, assume $\operatorname{diam}(X)<$ $\frac{1}{4} \operatorname{diam}(\Lambda)$, let $s=\frac{1}{4} N(\Lambda)$ and write $h^{-}$for $h_{s}$ of Lemma E.2. Note that $\mathfrak{h}^{-} \uparrow \mathfrak{h}$ as desired. By the second bound in Lemma $\mathrm{E} .2$, for a test function $f \in \Phi(X, \mathfrak{h})$,

$$
\left|\langle F, f\rangle_{\phi_{\iota}}\right|=\left|\left\langle\iota F, f_{\iota^{-1}}\right\rangle_{\phi}\right| \leq\|\iota F\|_{T_{\phi}(\mathfrak{h})}\left\|f_{\iota^{-1}}\right\|_{\Phi(\iota X, \mathfrak{h})} \leq\|\iota F\|_{T_{\phi}(\mathfrak{h})}\|f\|_{\Phi\left(X, \mathfrak{h}^{-}\right)} .
$$

Taking the supremum over $f$ with $\|f\|_{\Phi\left(X, \mathfrak{h}^{-}\right)}=1$, we have $\|F\|_{T_{\phi_{\iota}}\left(\mathfrak{h}^{-}\right)} \leq\|\iota F\|_{T_{\phi}(\mathfrak{h})}$, which completes the proof.

Lemma E.4. Let $X \in \mathcal{P}_{k}\left(\mathbb{Z}^{d}\right), F \in \mathcal{N}\left(X^{\square}\right)$, and let $\iota: \tilde{X} \rightarrow \Lambda$ be a coordinate map with $X_{N / 4} \subset \tilde{X} \in \mathcal{P}_{k}\left(\mathbb{Z}^{d}\right)$. The map $F \mapsto \iota F$ is a linear map from $\mathcal{N}\left(X^{\square}\right)$ to $\mathcal{N}\left(\iota X^{\square}\right)$, and obeys

$$
\|\iota F\|_{\mathcal{G}} \leq\|F\|_{\mathcal{G}}
$$

for either choice of the regulators $\mathcal{G}=G$ or $\mathcal{G}=\tilde{G}$ (recall (1.35)-(1.36) $)$.

Proof. The linearity of $F \mapsto \iota F$ is clear. By the definition of the norm, followed by Lemma E.3 and then Lemma E.2 (to bound the norm in the regulator),

$$
\begin{gathered}
\|\iota F\|_{\mathcal{G}}=\sup _{\phi \in \mathbb{C}^{\Lambda}}\|\iota F\|_{T_{\phi}} \mathcal{G}^{-1}(\iota X, \phi) \leq \sup _{\phi \in \mathbb{C}^{\Lambda}}\|F\|_{T_{\phi_{\iota}}} \mathcal{G}^{-1}(\iota X, \phi) \\
\leq \sup _{\phi \in \mathbb{C}^{\Lambda}}\|F\|_{T_{\phi_{\iota}}} \mathcal{G}^{-1}\left(X, \phi_{\iota}\right) \leq\|F\|_{\mathcal{G}},
\end{gathered}
$$

and the proof is complete.

Lemma E.5. Let $\mathfrak{h}^{-}$be as in LemmaE. E.3, and let $\gamma^{+}=\gamma\left(\mathfrak{h} / \mathfrak{h}^{-}\right)^{2}$. For $X \in \mathcal{P}_{k}\left(\mathbb{Z}^{d}\right), F \in \mathcal{N}\left(X^{\square}\right)$, $\gamma \in(0,1]$, and for a coordinate map $\iota: \tilde{X} \rightarrow \Lambda$ with $X_{N / 4} \subset \tilde{X} \in \mathcal{P}_{k}\left(\mathbb{Z}^{d}\right)$,

$$
\begin{aligned}
& \|F\|_{T_{0}\left(\mathfrak{h}^{-}\right)} \leq\|\iota F\|_{T_{0}(\mathfrak{h})} \\
& \|F\|_{\tilde{G}^{\gamma^{+}\left(\mathfrak{h}^{-}\right)}} \leq\|\iota F\|_{\tilde{G}^{\gamma}(\mathfrak{h})} .
\end{aligned}
$$

Proof. We only prove (E.14), because (E.13) is a specialisation of the same method to $\phi=0$. Let $\phi \in \mathbb{C}^{\Lambda}$. By Lemma E.2.

$$
\|\phi\|_{\Phi(\iota X, \mathfrak{h})} \leq\left\|\phi_{\iota}\right\|_{\Phi\left(X, \mathfrak{h}^{-}\right)}=\left(\mathfrak{h} / \mathfrak{h}^{-}\right)\left\|\phi_{\iota}\right\|_{\Phi(X, \mathfrak{h})},
$$


and hence, by definition of the regulator, $\tilde{G}^{\gamma}(\iota X, \phi) \leq \tilde{G}^{\gamma^{+}}\left(X, \phi_{\iota}\right)$. Therefore, by Lemma E.3,

$$
\|F\|_{\left.T_{\phi_{\iota}(\mathfrak{h}}\right)} \leq\|\iota F\|_{T_{\phi}(\mathfrak{h})} \leq\|\iota F\|_{T_{\phi}(\mathfrak{h})} \tilde{G}^{-\gamma}(\iota X, \phi) \tilde{G}^{\gamma^{+}}\left(X, \phi_{\iota}\right)
$$

We divide by $\tilde{G}^{\gamma^{+}}$and take the the supremum over $\phi$ to complete the proof.

Let $\mathcal{X} \subset \mathcal{C}_{k}(\mathbb{V})$, and let $F: \mathcal{X} \rightarrow \mathcal{N}$ have the properties listed in Definition 1.7 except that Euclidean covariance is replaced by the restricted version that if $X, Y \in \mathcal{X}$ and $E$ is a Euclidean automorphism such that $Y=E X$ then $E(F(X))=F(E X)$. Let $W: \mathcal{C}_{k}(\mathbb{V}) \rightarrow \mathbb{R}_{+}$be a Euclidean invariant function such that $\|F(X)\|_{k} \leq W(X)$ for $X \in \mathcal{C}(U)$. The following lemma, whose proof does not depend on the other lemmas in this appendix, shows that $F$ has an extension to an element of $\mathcal{K}(\mathbb{V})$.

Lemma E.6. Any $F: \mathcal{X} \rightarrow \mathcal{N}$ as above has an extension to an element $\hat{F} \in \mathcal{K}(\mathbb{V})$ such that $\|\hat{F}(X)\|_{k} \leq W(X)$ for $X \in \mathcal{C}(\mathbb{V})$. The map $F \mapsto \hat{F}$ is linear, and if $F(X)$ satisfies (1.37) for $X$ in $\mathcal{X}$, then the same is true for $\hat{F}(X)$ for all polymers $X$.

Proof. For $X \in \mathcal{C}_{k}(\mathbb{V})$ such that $X=E Y$ for some automorphism $E$ of $\mathbb{V}$ and some $Y \in \mathcal{X}$, define $\hat{F}(X)=E F(Y)$. If there exists another $Y^{\prime} \in \mathcal{X}$ and an automorphism $E^{\prime}$ such that $X=E^{\prime} Y^{\prime}$, then $A=E^{-1} E^{\prime}$ is a Euclidean automorphism such that $A Y^{\prime}=Y$. By hypothesis, $E^{\prime} F\left(Y^{\prime}\right)=E A F\left(Y^{\prime}\right)=E F\left(A Y^{\prime}\right)=E F(Y)$, so this definition of $\hat{F}$ is not dependent on choices. If there is no pair $Y, E$ such that $X=E Y$ then define $\hat{F}(X)=0$. By construction $\hat{F}$ has the properties listed in Definition 1.7, the extension is bounded by $W$, linear in $F$, and preserves the property (1.37).

\section{F Aspects of symmetry}

We now prove properties of the polynomial $Q$ of (1.70), and prove in particular that it lies in $\mathcal{Q}$ as claimed below (1.70). In addition, we prove that Gaussian expectation preserves defining properties of the space $\mathcal{K}$ in Definition 1.7, this is used in the proof of Proposition 5.1.

We draw attention to a notational clash in this appendix: $Q$ denotes the polynomial (1.70) in Lemma F.2, whereas $Q$ denotes the supersymmetry generator (see [7, Section 5.2.1] or [17, Section 6]) in Lemma F.3.

For the following lemma, we write $\left.F\right|_{0}$ for the constant part of $F \in \mathcal{N}$, which results from setting $\phi=0$ and $\psi=0$ in $F$.

Lemma F.1. For $F \in \mathcal{N}$ and $X \subset \Lambda$, the constant monomial of $\operatorname{Loc}_{X} F$ is $\left.F\right|_{0}$.

Proof. It is the defining property of $\operatorname{Loc}_{X} F$ in [19, Definition 1.6] that $\langle F, g\rangle_{0}=\left\langle\operatorname{Loc}_{X} F, g\right\rangle_{0}$ for all test functions $g$ in the space $\Pi$ of polynomial test functions. One such test function is $g_{\varnothing}=1$ (a test function with no arguments). By setting $g=g_{\varnothing}$ in the pairing, we obtain $\left.F\right|_{0}=\left.\left(\operatorname{Loc}_{X} F\right)\right|_{0}$. Since $\left.\left(\operatorname{Loc}_{X} F\right)\right|_{0}$ is the constant monomial of $\operatorname{Loc}_{X} F$, the proof is complete.

Lemma F.2. The formula $Q(B)=\sum_{Y \in \mathcal{S}(\Lambda): Y \supset B} \operatorname{Loc}_{Y, B} I^{-Y} K(Y)$ of (1.70) defines an element $Q \in \mathcal{Q}$. 
Proof. The operator Loc preserves Euclidean covariance, gauge invariance, and supersymmetry, according to [19, Proposition 1.9] and [19, Proposition 1.14]. Since $V$ and $K$ have these properties, therefore $Q$ also has them. It is then a consequence of [7, Lemma 5.3] that $Q$ lies in $\mathcal{Q}$, once we prove that $Q$ cannot have a constant term. But by Lemma F.1, the constant monomial in $\operatorname{Loc}_{Y} I^{-Y} K(Y)$ equals the constant part of $I^{-Y} K(Y)$, and this is zero by the assumption that $K \in \mathcal{K}$ and $V \in \mathcal{Q}$. Therefore, the constant monomial in $\operatorname{Loc}_{Y, B} I^{-Y} K(Y)$ is also zero, and hence so is the constant monomial in $Q(B)$, as desired.

To understand the role of the block $B$ in more detail, we first note that any choice of $B$ determines $\pi_{\varnothing} Q$, because by the Euclidean invariance of $\pi_{\varnothing} K$ specified in Definition 1.7, (1.70) assigns the same value to $\pi_{\varnothing} Q$ for all choices of $B$. For the observable terms, because (1.9) contains indicator functions, (1.70) does not determine the coupling constants in $\pi_{\alpha} Q(\alpha=a, b, a b)$ unless $B$ contains $a$ or $b$. Taking all choices of $B$, (1.70) consistently determines a unique element $Q$ in $\mathcal{Q}$.

Lemma F.3. Let $F \in \mathcal{N}$ and suppose that $\mathbb{E}_{j+1} \theta F$ exists.

(i) If $F$ is gauge invariant or Euclidean covariant, then so is $\mathbb{E}_{j+1} \theta F$.

(ii) The supersymmetry generator $Q$ commutes with $\mathbb{E}_{j+1} \theta$, i.e., $Q \mathbb{E}_{j+1} \theta=\mathbb{E}_{j+1} \theta Q$. In particular, if $F$ is supersymmetric then so is $\mathbb{E}_{j+1} \theta F$.

(iii) If $F$ is supersymmetric, then $\mathbb{E}_{j+1} F=\left.F\right|_{0}$. In particular, if $F$ has zero constant part, then so does $\mathbb{E}_{j+1} \theta F$.

Proof. Throughout the proof, we write simply $\mathbb{E}$ for $\mathbb{E}_{j+1}$, and we omit some details. All forms in the proof have even degree.

(i) Let $A_{j+1}=C_{j+1}^{-1}$. By definition,

$$
(\mathbb{E} \theta F)(\sigma, \bar{\sigma}, \phi, \bar{\phi}, \psi, \bar{\psi})=\int e^{-S_{A_{j+1}}(\xi, \bar{\xi}, \eta, \bar{\eta})} F(\sigma, \bar{\sigma}, \phi+\xi, \bar{\phi}+\bar{\xi}, \psi+\eta, \bar{\psi}+\bar{\eta}),
$$

where the action $S_{A_{j+1}}$ is Euclidean and gauge invariant. The claim can be seen to follow from this.

(ii) From [7, (5.13)-(5.14)], we know that $\hat{Q}=(2 \pi i)^{-1 / 2} Q$ commutes with $\mathcal{L}$ and hence also with $e^{\mathcal{L}}$. Since the action of $\mathbb{E} \theta$ on polynomials is the same as the action of $e^{\mathcal{L}}$ by [18, Lemma 4.2], this implies that $\mathbb{E} \theta Q P=Q \mathbb{E} \theta P$ for polynomials $P \in \mathcal{N}$.

A proof for general integrable elements of $\mathcal{N}$ can be based on the argument of [16, Lemma A.2], and we provide a sketch. By definition, $\theta F$ is a function of fluctuation and other fields, and the expectation integrates out the fluctuation fields leaving dependence on the others. We denote integration with respect to the fluctuation fields by $\int_{1}$, with respect to the other fields by $\int_{2}$, and with respect to all fields by $\int_{21}$. Then for a form $K_{12}$ depending on both fields, and a form $K_{2}$ depending on the other fields, since the integral of any $Q$-exact form is zero (see [17, p.58]), we have $\int_{21} Q\left(K_{2} K_{12}\right)=0$. Therefore, since $Q$ is an antiderivation and $K_{2}$ has even degree,

$$
\int_{2} K_{2} \int_{1} Q K_{12}=\int_{21} K_{2}\left(Q K_{12}\right)=-\int_{21}\left(Q K_{2}\right) K_{12}=-\int_{2}\left(Q K_{2}\right) \int_{1} K_{12} .
$$

Similarly, $\int_{2} Q\left(K_{2} \int_{1} K_{12}\right)=0$, and hence $\int_{2}\left(Q K_{2}\right) \int_{1} K_{12}=-\int_{2} K_{2} Q \int_{1} K_{12}$. Thus we have shown that

$$
\int_{2} K_{2} \int_{1} Q K_{12}=\int_{2} K_{2} Q \int_{1} K_{12} .
$$


Since $K_{2}$ is arbitrary, this implies that $Q \int_{1} K_{12}=\int_{1} Q K_{12}$.

We set $K_{12}=e^{-S_{A}} \theta F$ and use $Q e^{-S_{A}}=0$ (for $A=C_{j+1}^{-1}$ ) to conclude that

$$
Q \mathbb{E} \theta F=Q \int e^{-S_{A}} \theta F=\int Q\left(e^{-S_{A}} \theta F\right)=\int e^{-S_{A}} Q \theta F=\mathbb{E} Q \theta F .
$$

In particular, $Q \mathbb{E} \theta F=\mathbb{E} Q \theta F$. It suffices finally to show that $Q \theta F=\theta Q F$. Since $Q$ is an antiderivation and $\theta$ is a homomorphism, it is enough to verify that $Q \theta F=\theta Q F$ for $F=f(\phi, \bar{\phi})$, $F=\psi_{x}$, and $F=\bar{\psi}_{x}$. These are readily verified using $Q=d+\iota_{X}$ (see [17, (6.4)]).

(iii) Suppose that $F$ is supersymmetric. Let $\tilde{F}=F-\left.F\right|_{0}$, which is supersymmetric and has zero constant part. For $m \geq 0$, let $\tilde{F}(m)=e^{-m \sum_{x \in \Lambda} \tau_{x}} \tilde{F}$. We claim that $\mathbb{E} \tilde{F}(m)$ is independent of $m$. Indeed, let $v_{x}=\phi_{x} \psi_{x}$. Then $\tau_{x}=\hat{Q} v_{x}$ and since $\hat{Q} \tilde{F}=0$,

$$
\frac{\partial}{\partial m} \mathbb{E} \tilde{F}(m)=\sum_{x \in \Lambda} \mathbb{E}\left(\tau_{x} \tilde{F}(m)\right)=\sum_{x \in \Lambda} \mathbb{E}\left(\hat{Q}\left(v_{x} \tilde{F}(m)\right)\right) .
$$

The right-hand side of (F.5) is zero, since the integral of any $Q$-exact form vanishes (see [17, p.58]). It follows that $\mathbb{E} \tilde{F}=\lim _{m \rightarrow \infty} \mathbb{E} \tilde{F}(m)$, and this limit vanishes since $\tilde{F}$ has zero constant part. Therefore, $\mathbb{E} F=\left.F\right|_{0}$. In particular, since $\left.(\mathbb{E} \theta F)\right|_{0}=\mathbb{E} F$, if $F$ has zero constant part then so does $\mathbb{E} \theta F$.

\section{Acknowledgements}

The work of both authors was supported in part by NSERC of Canada. DB gratefully acknowledges the support and hospitality of the Institute for Advanced Study at Princeton and of Eurandom during part of this work. GS gratefully acknowledges the support and hospitality of the Institut Henri Poincaré, and of the Kyoto University Global COE Program in Mathematics, during visits to Paris and Kyoto where part of this work was done. We thank Roland Bauerschmidt for numerous helpful discussions and valuable advice, and Benjamin Wallace for corrections to a previous version.

\section{References}

[1] A. Abdesselam, A. Chandra, and G. Guadagni. Rigorous quantum field theory functional integrals over the $p$-adics I: Anomalous dimensions. Preprint, (2013).

[2] S. Adams, R. Kotecký, and S. Müller. Strict convexity of the surface tension for non-convex potentials. Preprint, (2012).

[3] T. Bałaban. Ultraviolet stability in field theory. The $\phi_{3}^{4}$ model. In J. Fröhlich, editor, Scaling and Self-Similarity in Physics. Birkhäuser, Boston, (1983).

[4] R. Bauerschmidt. A simple method for finite range decomposition of quadratic forms and Gaussian fields. Probab. Theory Related Fields, 157:817-845, (2013). 
[5] R. Bauerschmidt, D.C. Brydges, and G. Slade. Critical two-point function of the 4-dimensional weakly self-avoiding walk. To appear in Commun. Math. Phys.

[6] R. Bauerschmidt, D.C. Brydges, and G. Slade. Logarithmic correction for the susceptibility of the 4-dimensional weakly self-avoiding walk: a renormalisation group analysis. To appear in Commun. Math. Phys.

[7] R. Bauerschmidt, D.C. Brydges, and G. Slade. A renormalisation group method. III. Perturbative analysis. To appear in J. Stat. Phys.

[8] R. Bauerschmidt, D.C. Brydges, and G. Slade. Structural stability of a dynamical system near a non-hyperbolic fixed point. Annales Henri Poincaré, doi:10.1007/s00023-014-0338-0.

[9] R. Bauerschmidt, D.C. Brydges, and G. Slade. Scaling limits and critical behaviour of the 4-dimensional $n$-component $|\varphi|^{4}$ spin model. J. Stat. Phys, 157:692-742, (2014).

[10] G. Benfatto, M. Cassandro, G. Gallavotti, F. Nicolò, E. Oliveri, E. Presutti, and E. Scacciatelli. Some probabilistic techniques in field theory. Commun. Math. Phys., 59:143-166, (1978).

[11] G. Benfatto, M. Cassandro, G. Gallavotti, F. Nicolò, E. Oliveri, E. Presutti, and E. Scacciatelli. On the ultraviolet stability in the Euclidean scalar field theories. Commun. Math. Phys., 71:95-130, (1980).

[12] G. Benfatto and G. Gallavotti. Renormalization Group. Princeton University Press, Princeton, N.J., (1995).

[13] D. Brydges, S.N. Evans, and J.Z. Imbrie. Self-avoiding walk on a hierarchical lattice in four dimensions. Ann. Probab., 20:82-124, (1992).

[14] D.C. Brydges. Lectures on the renormalisation group. In S. Sheffield and T. Spencer, editors, Statistical Mechanics, pages 7-93. American Mathematical Society, Providence, (2009). IAS/Park City Mathematics Series, Volume 16.

[15] D.C. Brydges, G. Guadagni, and P.K. Mitter. Finite range decomposition of Gaussian processes. J. Stat. Phys., 115:415-449, (2004).

[16] D.C. Brydges and J.Z. Imbrie. Green's function for a hierarchical self-avoiding walk in four dimensions. Commun. Math. Phys., 239:549-584, (2003).

[17] D.C. Brydges, J.Z. Imbrie, and G. Slade. Functional integral representations for self-avoiding walk. Probab. Surveys, 6:34-61, (2009).

[18] D.C. Brydges and G. Slade. A renormalisation group method. I. Gaussian integration and normed algebras. To appear in J. Stat. Phys.

[19] D.C. Brydges and G. Slade. A renormalisation group method. II. Approximation by local polynomials. To appear in J. Stat. Phys. 
[20] D.C. Brydges and G. Slade. A renormalisation group method. IV. Stability analysis. To appear in J. Stat. Phys.

[21] D.C. Brydges and H.-T. Yau. Grad $\phi$ perturbations of massless Gaussian fields. Commun. Math. Phys., 129:351-392, (1990).

[22] S.B. Chae. Holomorphy and Calculus in Normed Spaces. Marcel Dekker, Inc., New York, (1985).

[23] J. Dimock. The renormalization group according to Bałaban I. Small fields. Reviews in Math. Phys., 25:1330010, (2013).

[24] J. Dimock and T.R. Hurd. A renormalization group analysis of correlation functions for the dipole gas. J. Stat. Phys., 66:1277-1318, (1992).

[25] P. Falco. Kosterlitz-Thouless transition line for the two dimensional Coulomb gas. Commun. Math. Phys., 312:559-609, (2012).

[26] P. Falco. Critical exponents of the two dimensional Coulomb gas at the Berezinskii-KosterlitzThouless transition. Preprint, (2013).

[27] J. Feldman, H. Knörrer, and E. Trubowitz. Fermionic Functional Integrals and the Renormalization Group. CRM Monograph Series, Volume 16. American Mathematical Society, Providence, (2002).

[28] J. Feldman, J. Magnen, V. Rivasseau, and R. Sénéor. Construction and Borel summability of infrared $\Phi_{4}^{4}$ by a phase space expansion. Commun. Math. Phys., 109:437-480, (1987).

[29] K. Gawędzki and A. Kupiainen. Block spin renormalization group for dipole gas and $(\nabla \varphi)^{4}$. Ann. Physics, 147:198-243, (1983).

[30] K. Gawędzki and A. Kupiainen. Lattice dipole gas and $(\nabla \varphi)^{4}$ models at long distances: Decay of correlations and scaling limit. Commun. Math. Phys., 92:531-553, (1984).

[31] K. Gawȩdzki and A. Kupiainen. Massless lattice $\varphi_{4}^{4}$ theory: Rigorous control of a renormalizable asymptotically free model. Commun. Math. Phys., 99:199-252, (1985).

[32] K. Gawȩdzki and A. Kupiainen. Asymptotic freedom beyond perturbation theory. In K. Osterwalder and R. Stora, editors, Critical Phenomena, Random Systems, Gauge Theories, Amsterdam, (1986). North-Holland. Les Houches 1984.

[33] V. Mastropietro. Non-Perturbative Renormalization. World Scientific, Singapore, (2008).

[34] P.K. Mitter and B. Scoppola. The global renormalization group trajectory in a critical supersymmetric field theory on the lattice $\mathbf{Z}^{3}$. J. Stat. Phys., 133:921-1011, (2008).

[35] J. Pöschel and E. Trubowitz. Inverse Spectral Theory, volume 130 of Pure and Applied Mathematics. Academic Press Inc., Boston, MA, (1987). 
[36] V. Rivasseau. From Perturbative to Constructive Renormalization. Princeton University Press, Princeton, N.J., (1991).

[37] V. Rivasseau and Z. Wang. Corrected loop vertex expansion for $\phi_{2}^{4}$ theory. Preprint, (2014).

[38] M. Salmhofer. Renormalization: An Introduction. Springer, Berlin, (1999).

[39] G. Slade and A. Tomberg. Critical correlation functions for the 4-dimensional weakly selfavoiding walk and $n$-component $|\varphi|^{4}$ model. Preprint, (2014).

[40] K.G. Wilson and J. Kogut. The renormalization group and the $\epsilon$ expansion. Phys. Rep., 12:75-200, (1974). 